\title{
A review of the genus Cordilura Fallén, 1810 (Diptera: Scathophagidae) of Russia
}

\section{Обзор видов рода Cordilura Fallén, 1810 (Diptera: Scathophagidae) фауны России}

\author{
A.L. Ozerov ${ }^{1^{*}}$, M.G. Krivosheina ${ }^{2}$ \\ А.А. Озеров ${ }^{1^{*}}$, М.Г. Кривошеина ${ }^{2}$
}

\author{
'Zoological Museum, Moscow Lomonosov State University, Bol'shaya Nikitskaya 2, Moscow 125009, Russia. \\ E-mail: ozerov2455@rambler.ru \\ 'Зоологический музей, Московский государственный университет им. М.В. Ломоносова, Большая Никитская ул., 2, Москва \\ 125009 , Россия. \\ ${ }^{2}$ A.N. Severtsov Institute of Ecology and Evolution, Russian Academy of Sciences, Leninsky prospect, 33, Moscow 119071, Russia. \\ E-mail: dipteramarina@rambler.ru \\ ${ }^{2}$ Институт проблем экологии и эволюции им. А.Н. Северцова РАН, Ленинский проспект, 33, Москва 119071, Россия. \\ *corresponding author
}

KEYWORDS: Diptera, Scathophagidae, dung flies, Cordilura, Russia, review, new species, new records.

КЛЮЧЕВЫЕ СЛОВА: Diptera, Scathophagidae, Cordilura, Russia, новый вид, новые данные по распространению.

ABSTRACT. Flies of the genus Cordilura Fallén, 1810 of Russia, which includes 38 species, are reviewed. Generic and species descriptions and key for determination of species are given, and data on distributions are summarized. One species, Cordilura nubilosa sp.n., is described as new to science. New records of the following species are given: Cordilura albilabris Fabricius, 1805 (Armenia, Kazakhstan, Kyrgyzstan); C. albipes Fallén, 1819 (Armenia, Belarus, Georgia); C. impudica Rondani, 1867 (Uzbekistan); C. pudica Meigen, 1826 (Belarus); C. rufipes Meigen, 1826 (Georgia, Kazakhstan).

РЕЗЮМЕ. Дан обзор двукрылых рода Cordilura Fallén, 1810 фауны России, включающего 38 видов. Приведены диагноз рода, описания видов и ключ для определения видов рода Cordilura Pocсии, а также суммированы данные по распространению видов рода на территории России. Описан один новый для науки вид, Cordilura nubilosa sp.n. Приведены новые данные о распространении следующих видов: Cordilura albilabris Fabricius, 1805 (Армения, Казахстан, Киргизия); C. albipes Fallén, 1819 (Армения, Белоруссия, Грузия); Cordilura impudica Rondani, 1867 (Узбекистан); C. pudica Meigen, 1826 (Белоруссия); C. rufipes Meigen, 1826 (Грузия, Казахстан).

\section{Introduction}

Cordilura Fallén, 1810 is the largest genus within the family Scathophagidae and consists of 91 species distributed in the Northern Hemisphere: 46 species are known in Palaearctic [Iwasa, 2020] and 36 in Nearctic [Vockeroth, 1965, 1987], with 7 species with Holarctic distribution; 2 species are recorded in Oriental Region [Ozerov, Krivosheina, 2012a; 2013].

The fauna of Russia includes 37 species [Loew, 1864; Becker, 1894; Hackman, 1956; Gorodkov, 1970, 1974, 1986; Elberg, 1972; Ozerov, 2007b, 2009; Šifner, 2008; Ozerov, Krivosheina, 2012b, 2015, 2017].

Faunistic reviews of some districts of Russia are published for the Kamchatka [Hendel, 1930; Ozerov, 2007a], Volga Region [Ovchinnikov, 2004], Karelia [Humala, Polevoi, 2009], Yakutia [Bagachanova et al., 2016] and Russian Far East [Ozerov, Krivosheina, 2014], but no general work has been fulfilled.

Almost all species of Cordilura whose habits are known are primary borers in culms of Carex and Scirpus spp. (Cyperaceae) [Neff, Wallace, 1969; Wallace, Neff, 1971]. Besides, larvae of Cordilura have been found in stems of Glyceria (Gramineae) and Juncus (Juncaceae) [Ferrar, 1987].

Adults of Cordilura species are often collected at locations where these plants grow: in marshy plots, at

How to cite this article: Ozerov A.L., Krivosheina M.G. 2020. A review of the genus Cordilura Fallén, 1810 (Diptera: Scathophagidae) of Russia // Russian Entomol. J. Vol.29. No.4. P.439-480. 10.15298/rusentj.29.4.13 

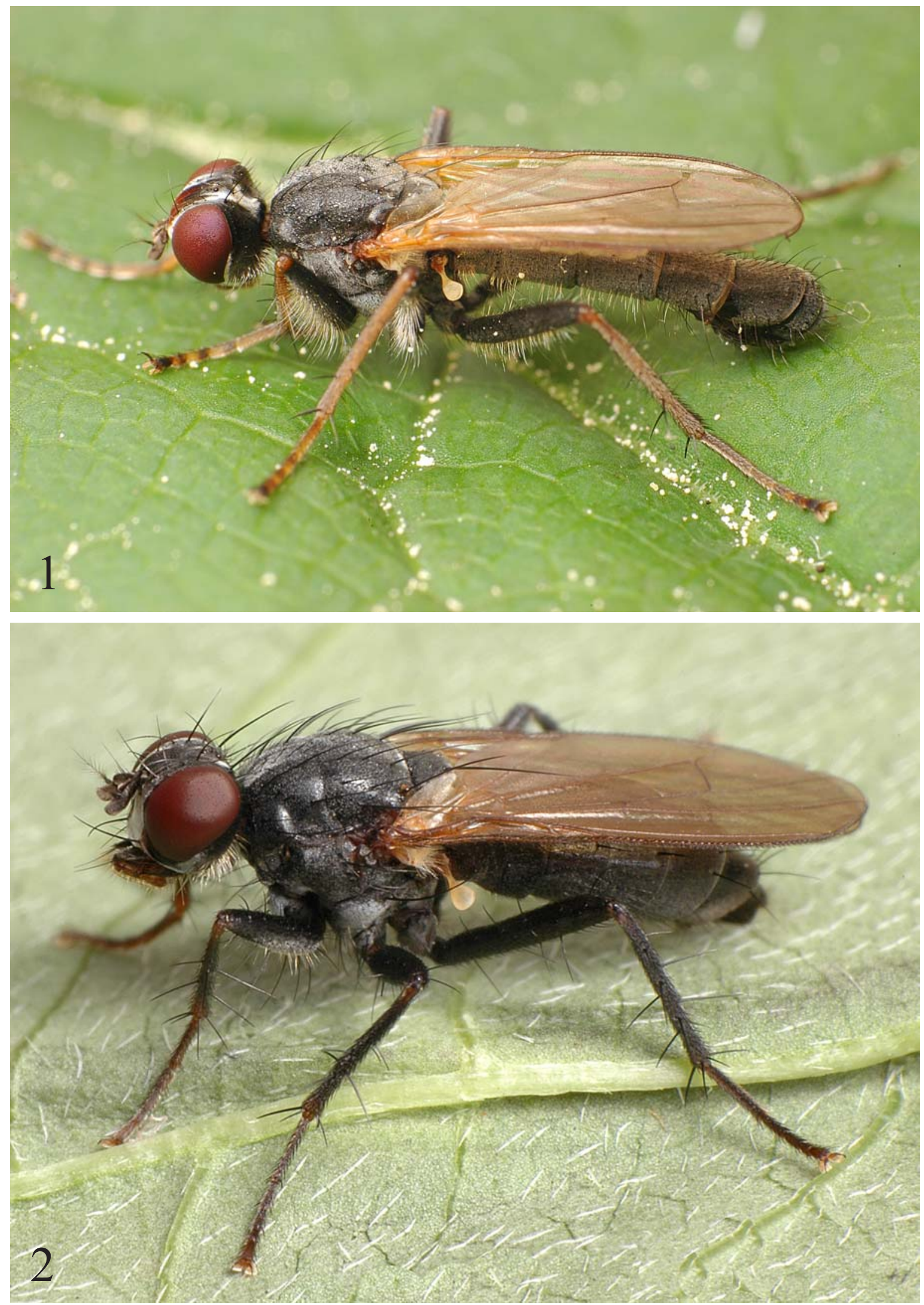

Figs 1-2. Male of Cordilura rufipes (Meigen) (1) and female of Cordilura picipes (Meigen) (2). Photographs by D.I. Gavryushin. Рис. 1-2. Самец Cordilura rufipes (Meigen) (1) и самка Cordilura picipes (Meigen) (2). Фото Д.И. Гаврюшина. 
banks of lakes covered with Carex spp., along spring banks and in other mesophytic habitats.

Life cycles, mature larvae, and puparia of $13 \mathrm{Ne}-$ arctic species of Cordilura are described [Neff, Wallace, 1969; Wallace, Neff, 1971]. Also 3rd stage larva and pupa of Holarctic species Cordilura pudica Meigen, 1826 is described [Wallace, Neff, 1971].

\section{Material and methods}

The specimens examined for this study are deposited in the Zoological Museum, Moscow State University, Russia (ZMUM) and Zoological Institute of Russian Academy of Sciences, St. Petersburg, Russia (ZISP). We also studied some material from the Institute of Systematics and Ecology of Animals, Russian Academy of Sciences, Siberian Branch, Novosibirsk, Russia (ISEA) and from Institute of Biology and Soil Sciences, Russian Academy of Sciences, Far Eastern Branch, Vladivostok, Russia (IBSS).

The majority of original Russian geographical names are given in transliteration, but names of some large geographical regions (e.g. Altai (=Altai Krai + Altai Republic), Krasnoyarsk Krai, Primorsky Krai, Sakha Republic (=Yakutia), Zabaykalsky Krai) follow these indicated in Google Earth software.

The data on distributions are cited as follows: Russia is in the first place, other countries of the world are given after a m-dash [-]. More detailed data on the distributions of species in Europe may be found in the work by Šifner [2008], and in North America in the work by Vockeroth [1965].

The terminology used in the generic and species descriptions follows McAlpine [1981]), Cumming \& Wood [2009], and Stuckenberg [1999].

\section{Taxonomic results}

\section{Cordilura Fallén, 1810}

Cordilura Fallén, 1810: 15. Gender: feminine. Type-species: Musca pubera Linnaeus, 1758 sensu Fallén, 1810 [misidentification, = Cordylura rufipes Meigen, 1826], by monotypy.

Phrosia Robineau-Desvoidy, 1830: 668. Gender: feminine. Type-species: Phrosia scirpi Robineau-Desvoidy, 1830, by monotypy [= Ocyptera albilabris Fabricius, 1805).

Mosina Robineau-Desvoidy, 1830: 670. Gender: feminine. Type-species: Musca pubera Linnaeus, 1758, by designation of Westwood, 1840.

Scoliaphleps Becker, 1894: 98. Gender: feminine. Type-species: Cordylura ustulata Zetterstedt, 1838, by original designation.

Cordilurina James, 1955: 96 [as subgenus of Cordilura]. Gender: feminine. Type-species: Cordylura vittipes Loew, by original designation [= Cordylura fuscipes Zetterstedt, 1838].

Cordylura: unjustified emend.

Adult Cordilura can be distinguished from the other genera of Scathophagidae by the following combination of characters: 1) katepisternum with one stong seta in posterodorsal corner, 2) palpus with strong apical (subapical) seta about as long as or longer than palpus, 3 ) fore femur and fore tibia without ventral rows of long spinous setae, 4) proepisternum is covered with hairs at middle or on anterior part.

Cordilura species are slender, small to mediumsized flies (about 3.5-10.5 mm long); color various, but usually is black (Figs 1,2).

Head subspherical (Fig. 7). Eyes moderately large, dichoptic in both sexes. Frontal vitta and fronto-orbital plate distinct. $1-3$ orbitals, $2-5$ frontals, 1 ocellar, 1 inner vertical, 1 outer vertical, 1 small postocellar setae present; 1 pair of strong vibrissae and 1-2 pairs of subvibrissae present. Postpedicel rounded apically, 1.5-3 times as long as wide, with arista nearly bare to plumose (Figs 3-7). Palpus slender, slightly broadened towards apex, with a long outstanding seta apically (Figs 8, 9).

Thorax as in most Calyptrata. Scutum with following setae: acrostichal usually setulose in two rows or absent, dorsocentrals $3+3$ (including scapular seta), intra-alars $(0-1)+(0-2)$, supra-alars $(0-1)+(1-2)$, postpronotals $1-2,2$ notopleurals, 2 postalars. Proepisternum covered with fine hairs and usually with 1-2 setae near ventral margin. Proepimeron with $0-1$ seta ventral to spiracle. Anepisternum with fine hairs in posterior half and 2-4 well-developed setae near posterior margin. Katepisternum with one long black seta in posterodorsal corner, ventrally and posteriorly with hairs. Anepimeron bare. Postmetacoxal bridge absent. Scutellum with one or two pairs of strong setae: with a pair of strong discal and a pair of strong apical setae (Fig. 13) or with a pair of strong discal setae and pair of apical setulae (Fig. 12).

Legs long and slender. Fore femur with a row of anterodorsal setae, with a row of thin and usually long ventral setae, sometimes with fine long hairs on posterior and posteroventral surfaces. Fore tibia with 1-2 dorsal/posterodorsal, 0-2 posterior, 0-2 anterodorsal, 2 posteroventral setae, and with a ring of apical setae. Mid femur usually with rows of anterior and anterodorsal setae and with 0-1 anterior apical, 0-2 posterior apical and $0-1$ posterodorsal apical setae. Mid tibia with 1-3 anterodorsal, 1-2 posterodorsal, 1-2 anteroventral, 0-2 posterior or posteroventral setae, and with a ring of apicals. Hind femur with a row of anterodorsal and several anteroventral setae, 0-2 dorsal setae in apical half, and 0-2 posterior apical setae. Hind tibia with 2-3 anterodorsal, 2-4 posterodorsal, 0-2 anterior or anteroventral, 1 dorsal preapical, 1 anterodorsal preapical, 1 posterodorsal preapical, 1 anteroventral apical, $0-1$ posteroventral apical and 0-1 ventral apical setae. Vockeroth [1987: 1090] considers species that lack posteroventral apical seta of hind tibia in the separate genus Bucephalina Malloch, 1919. We found another difference between Bucephalina and Cordilura in the structure of ovipositor (see below). Nevertheless we consider in this work all Russian species hind tibia of which lack posteroventral apical seta (C. amurensis Ozerov, 2007, C. krocha Ozerov, 2007, C. nartshukae Ozerov et Krivosheina, 2015, C. nubilosa sp.n., C. sifneri Ozerov, 2007, C. tartariana Ozerov, 2007) in the genus Cordilura. Molecular analysis based on DNA sequences for these genera may solve this issue in future. 

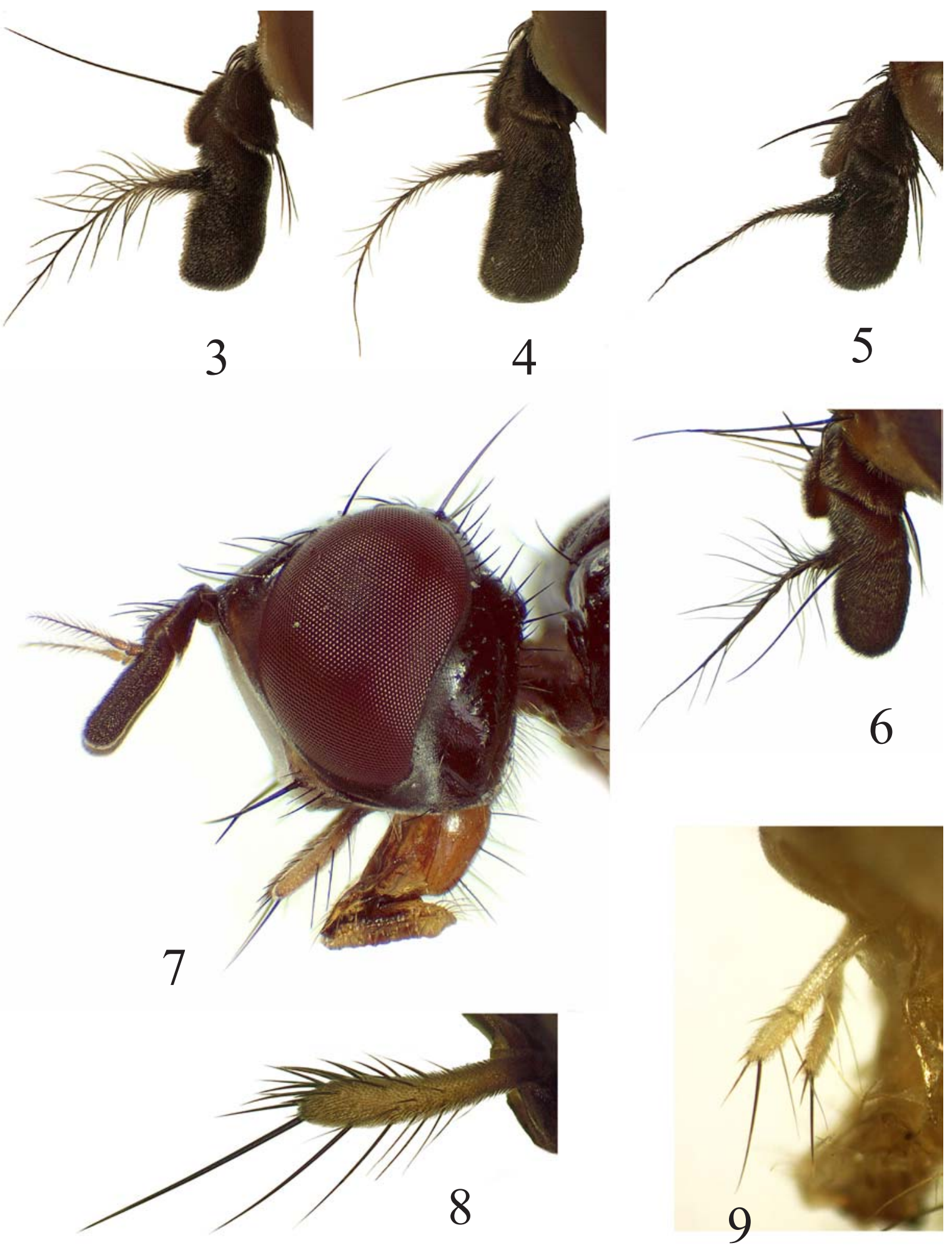

Figs 3-9. Cordilura spp.: 3 - antenna of C. rufipes (Meigen), lateral view; 4 - same of C. aemula (Collin); 5 - same of C. ustulata (Zetterstedt); 6 - same of C. ciliata (Meigen); 7 - head of C. albilabris (Fabricius), lateral view; 8 - palpus of C.remmi (Elberg); 16 same of $C$. sifneri Ozerov.

Рис. 3-9. Cordilura spp.: 3 - усик C. rufipes (Meigen), сбоку; 4 - то же у C. aemula (Collin); 5 - то же у C. ustulata (Zetterstedt); 6 - то же у C. ciliata (Meigen); 7 — голова C. albilabris (Fabricius), сбоку; 8 — щупик C.remmi (Elberg); 16 - то же у C. sifneri Ozerov. 

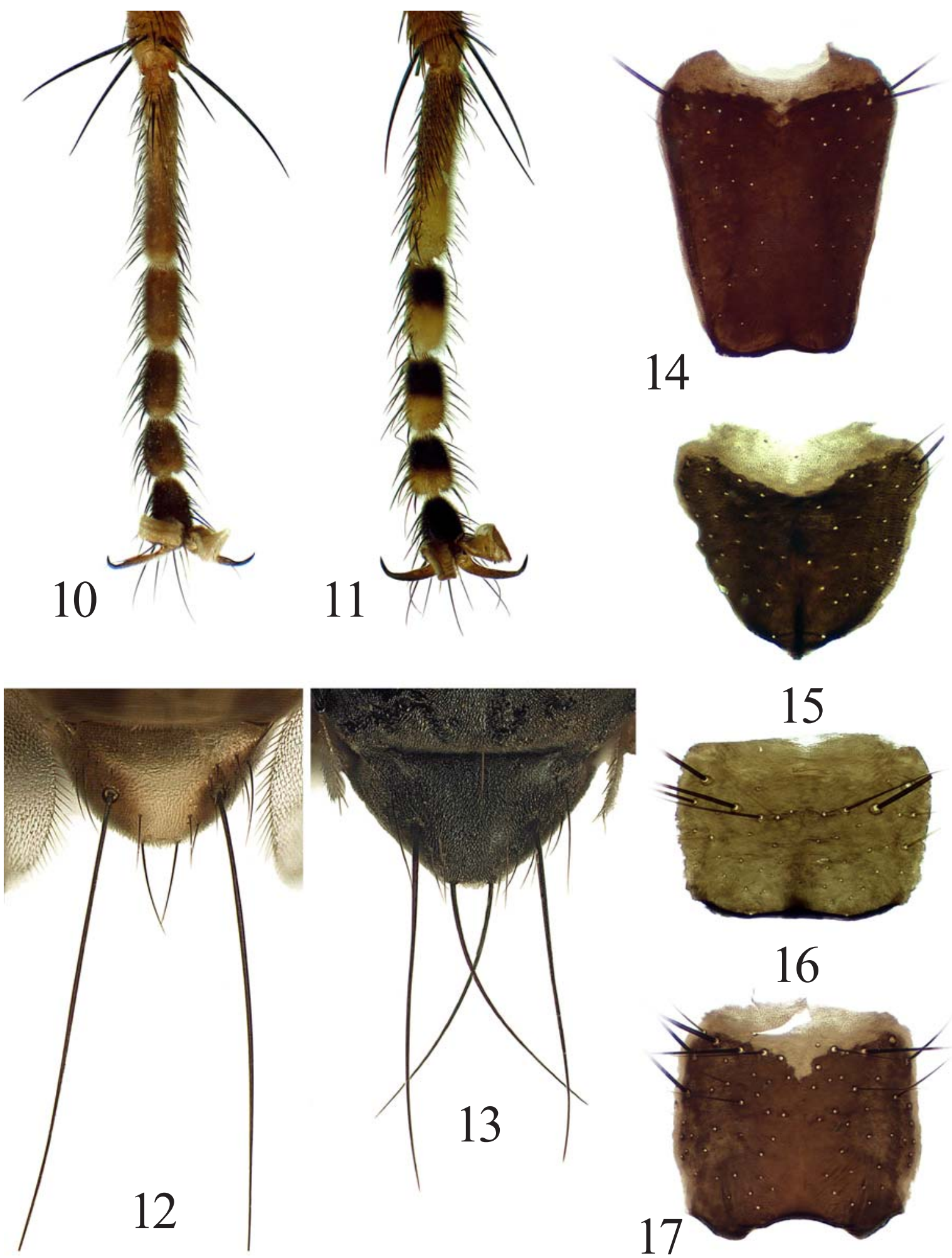

Figs 10-17. Cordilura spp.: 10 - fore tarsus of C. monochroma Ozerov et Krivosheina, ventral view; 11 - fore tarsus of C. rufipes (Meigen), ventral view; 12 - scutellum of $C$. albipes (Fallén), dorsally; 13 - scutellum of $C$. pudica (Meigen), dorsally; 14 - male sternite 4 of C. rufipes (Meigen); 15 - male sternite 4 of C. zaitzevi Gorodkov; 16 - male sternite 4 of C. proboscidea (Zetterstedt); 17 male sternite 4 of C.rufimana (Meigen). 10, 11 - after Ozerov \& Krivosheina, 2014, figs 14, 17.

Рис. 10-17. Cordilura spp.: 10 - лапка передней ноги C. monochroma Ozerov et Krivosheina, снизу; 11 - лапка передней ноги C. rufipes (Meigen), снизу; 12 - щиток C. albipes (Fallén), сверху; 13 - щиток C. pudica (Meigen), сверху; 14 - стернит 4 самца $C$. rufipes (Meigen); 15 - стернит 4 самца C. zaitzevi Gorodkov; 16 - стернит 4 самца C. proboscidea (Zetterstedt); 17 - стернит 4 самца C.rufimana (Meigen). 10, 11 — по Ozerov \& Krivosheina, 2014, figs 14, 17. 


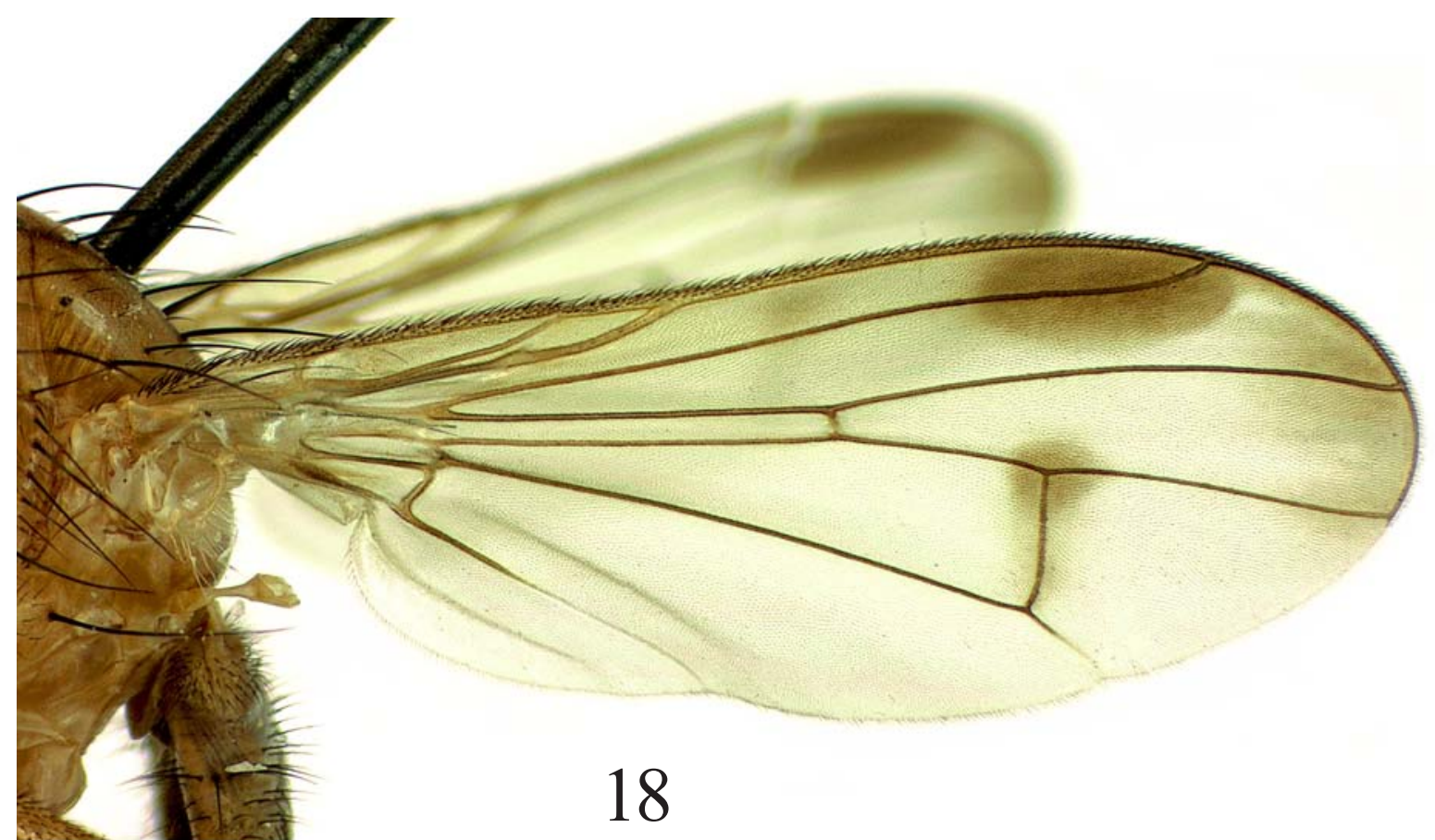

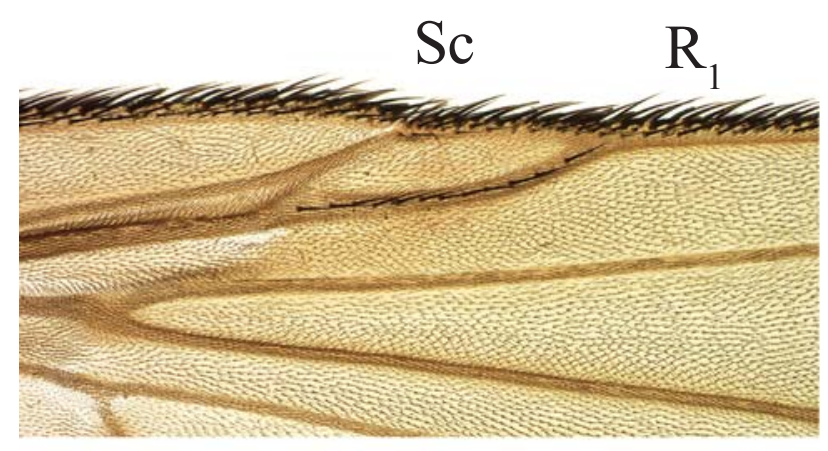

19

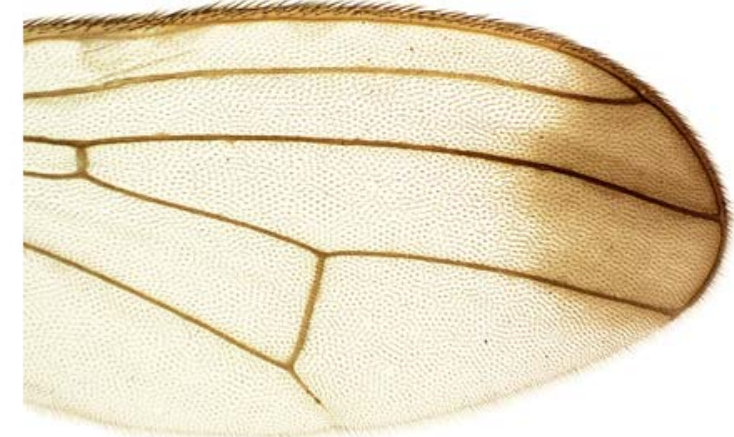

20

Figs 18-20. Wing of Cordilura spp.: $18-$ C. nartshukae Ozerov et Krivosheina; $19-$ C. impudica (Rondani), fragment; $20-C$. shatalkini Ozerov, apical part. 18 - after Ozerov \& Krivosheina, 2015, fig. 1.

Рис. 18-20. Крыло Cordilura spp.: $18-$ C. nartshukae Ozerov et Krivosheina; $19-$ C. impudica (Rondani), фрагмент; $20-C$. shatalkini Ozerov, апикальная часть. 18 - по Ozerov \& Krivosheina, 2015, fig. 1.

Wing well-developed, clear or tinged with brownish, rarely darkened apically (Fig. 20), rare with dark spots (Fig. 18). Vein $\mathrm{R}_{1}$ setulose on apical third of dorsal surface (Fig. 19) or bare.

Abdomen long, subcylindrical. Usually syntergite 1+2 with 2-3 lateral discal and 2-4 lateral marginal setae; tergites 3-6 each with a row of marginal setae. Male sternite 4 triangular, rectangular or trapezoid, as wide as long or wider than long (Figs 14-17). Sternite 5 with well developed lateral lobes from narrow (e.g., Figs 66, 93) to wide (e.g., Figs 72, 131). Surstyli symmetrical, simple (e.g., Figs 37, 102) or bifurcate (e.g., Figs 25,58$)$; cerci fused apically or medially forming cercal plate.
Ovipositor (Figs 21-23) long and compressed, with large membranous areas between segments. Tergite 7 sclerotized laterally and membranous dorsally. Sternite 6 well sclerotized, with apodeme of various lenght basally fused to sternite (Figs 22, 23) or without apodeme in most species. Sternite 7 poorly sclerotized medially, with long apodeme not fused to sternite 7 . In species $C$. krocha, $C$. nartshukae, C. amurensis, C. sifneri, C. tartariana sternite 7 without apodeme. Tergite 8 in form of heavily sclerotized sclerite with deep membranous dorsal emargination in distal part. Sternite 8 divided medially into 2 small, heavily sclerotized spatulate sclerites. Proctiger situated distally and represented by small entire epiproct, 1 pair of small cerci, and hypoproct. 

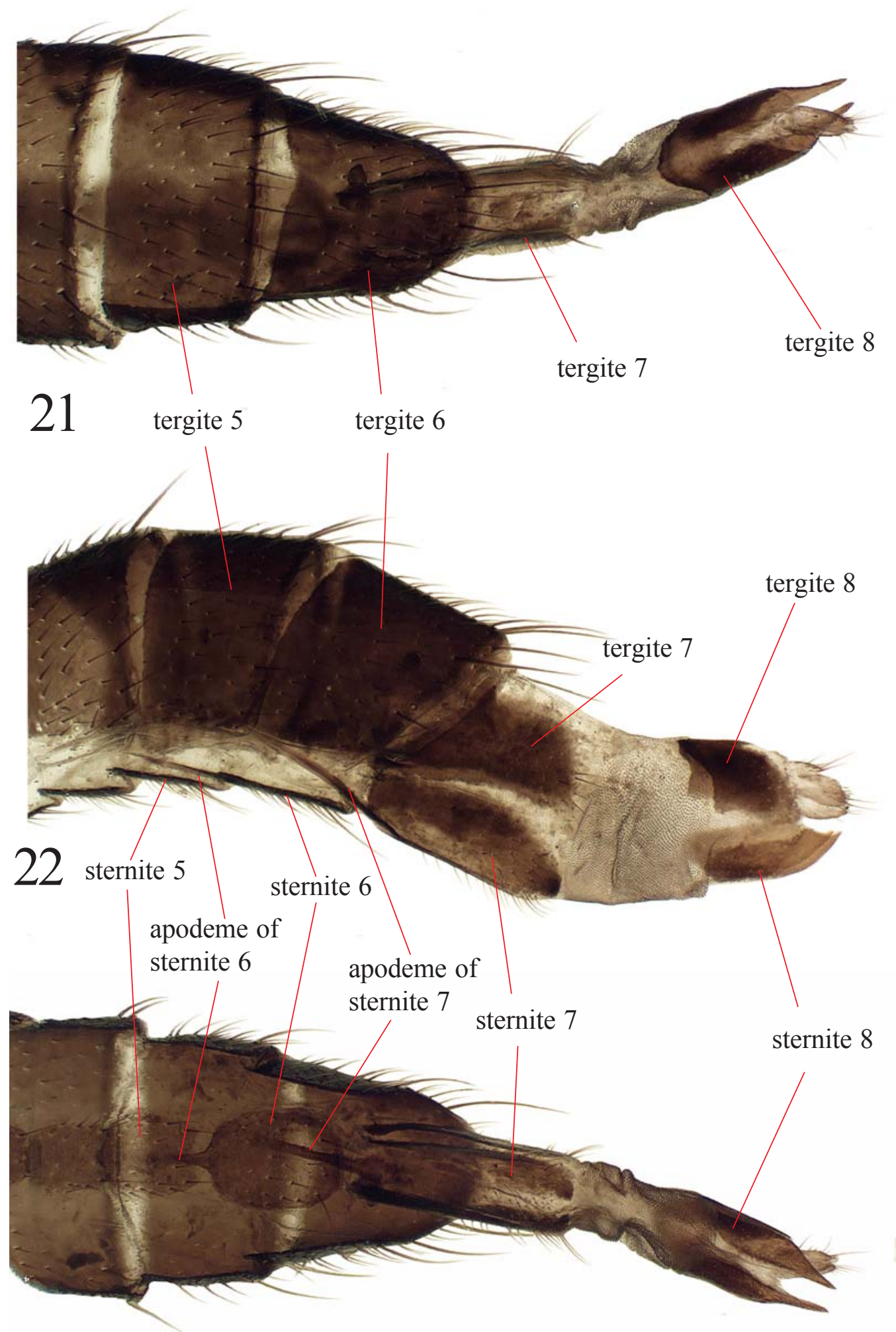

23

Figs 21-23. Cordilura albipes (Fallén): 21 - end of female abdomen, dorsal view; 22 - same, lateral view; 23 - same, ventral view. Pис 21-23. Cordilura albipes (Fallén): 21 - конец брюшка самки, сверху; 22 - то же, сбоку; 23 — то же, снизу. 
Cordilura aberrans (Becker, 1894)

Figs 24-26.

aberrans Becker, 1894: 91 (Cordylura). Type-locality: "Lapponia" (Sweden).

rubifrontata Becker, 1894: 91 (Cordylura). Type-locality: "Kultuk" (Russia, Baikal Area).

REMARK. This species was recorded in Russia for European part (Kola Peninsula, Bashkiria) by Gorodkov [1970: 446], for Siberia [Becker, 1894: 91 (as $C$. rubifrontata); Gorodkov, 1970: 446, 1986: 14 (without indicating specific locality)], for Far East (including Khabarovsk Krai and Magadan Oblast) [Ozerov, Krivosheina, 2014: 206] and for Yakutia [Bagachanova et al., 2016: 778].

MATERIAL EXAMINED. Altai: Artybash env. $\left(51.796^{\circ} \mathrm{N}\right.$ 87.265 $\left.{ }^{\circ} \mathrm{E}\right), 16-18$. VII.2006, Nartshuk (1 $\sigma^{7}$, ZISP); Cherga, $463 \mathrm{~m}$, $\left(51.57^{\circ} \mathrm{N} 85.56^{\circ} \mathrm{E}\right), 28$. VI.2009, A. Barkalov, V. Sorokina ( $1 \sigma^{7}, 1$, ISEA); Shebalino, floodplain of the River Sema, $462 \mathrm{~m},\left(51.57^{\circ} \mathrm{N}\right.$, $\left.85.58^{\circ} \mathrm{E}\right), 27-30 . \mathrm{VI} .2009$, V. Sorokina (2 $\sigma^{\top} \sigma^{\top}$, ISEA); Onguday

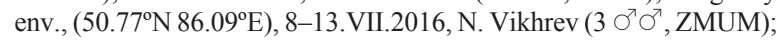
Seminsky Pass, River Sarlyk $\left(51.11^{\circ} \mathrm{N} 85.60^{\circ} \mathrm{E}\right), 1200 \mathrm{~m}, 28$ 30.VI.2016, N. Vikhrev ( $8 \sigma^{7} \sigma^{\top}, 1$ ㅇ, ZMUM); Seminsky Pass, River Turala $\left(50.99^{\circ} \mathrm{N} 85.68^{\circ} \mathrm{E}\right), 1350 \mathrm{~m}, 8-12 . V I I .2016$, N. Vikhrev (1 $\sigma^{7}$, ZMUM); $10 \mathrm{~km}$ E of Cheke-Taman Pass, River Bol'shoy Il'gumen' $\left(50.613^{\circ} \mathrm{N} 86.469^{\circ} \mathrm{E}\right), 28 . V I I .2006$, A. Ovchinnikov (2 $\Im^{7} \sigma^{7}, 4$ 우, ZISP); Turochak env. (52.261 $\left.{ }^{\circ} \mathrm{N} 87.123^{\circ} \mathrm{E}\right), 17 . \mathrm{VII} .2006$, A. Ovchinnikov (1 $\sigma^{7}$, ZISP); Amur Oblast: Komsomol'sk-naAmure, Silinskiy park $\left(50.573^{\circ} \mathrm{N} 137.039^{\circ} \mathrm{E}\right), 29 . \mathrm{V} .1985$, Mutin (1 +, IBSS); Zeya Town $\left(53.748^{\circ} \mathrm{N} 127.261^{\circ} \mathrm{E}\right), 12$.VII.1982, A. Ozerov (1 $\sigma^{7}, 10$ 우, ZMUM); Skovorodino $\left(53.986^{\circ} \mathrm{N} 123.943^{\circ} \mathrm{E}\right), 1-$ 6.VII.1929, Kuznetsov (1 ア , ZISP); $52 \mathrm{~km} \mathrm{~N}$ of Zeya Town $\left(54.087^{\circ} \mathrm{N}, 126.871^{\circ} \mathrm{E}\right), 1$. VII.1982, V. Zlobin (1 $\left.\sigma^{7}, \mathrm{ZISP}\right) ;$ Arkhangelsk Oblast: Nenetsky Nature Reserve, cordon "Bol'shoy Gusinets" (68.175 $\left.{ }^{\circ} \mathrm{N} 53.645^{\circ} \mathrm{E}\right), 9$. VII.2008, A.L. Ozerov (1 $\sigma^{7}$, ZMUM); Vas'kovo $\left(64.413^{\circ} \mathrm{N} 40.457^{\circ} \mathrm{E}\right), 8 . \mathrm{VII} .1996$, Gorodkov (1 9 , ZISP); Bashkiria: E of Kryktytau $\left(53.525^{\circ} \mathrm{N} 58.498^{\circ} \mathrm{E}\right), 2-8$.VIII.2008, K Tomkovich (2 90, ZMUM); Buryatia: the upper reaches of the River Irkut near Lake Il'chir $\left(51.961^{\circ} \mathrm{N} 100.952^{\circ} \mathrm{E}\right), 7$. VIII.2012, A Medvedev (1 $\sigma^{7}, 1$, ZMUM); Barguzinsky Nature Reserve (ca. $\left.54.35^{\circ} \mathrm{N} 109.51^{\circ} \mathrm{E}\right), 20 . \mathrm{VII} .1965$, Negrobov (1 $\sigma^{7}, 1$ ㅇ, ZISP); Khamney $\left(50.402^{\circ} \mathrm{N} 103.868^{\circ} \mathrm{E}\right), 29 . \mathrm{VI} .1971$, V. Richter (1 \%, ZISP); Selenduma $\left(50.916^{\circ} \mathrm{N} 106.239^{\circ} \mathrm{E}\right), 25 . \mathrm{VI} .1971$, V. Richter $(1$ o ZISP); Mondy env. $\left(51.675^{\circ} \mathrm{N} 100.992^{\circ} \mathrm{E}\right), 15$.VII.1965, Gorodkov (22 $\mathrm{O}^{7} \mathrm{O}^{7}, 18$ 웅, ZISP); Khargun, $6 \mathrm{~km} \mathrm{E}$ of Kyren $\left(51.683^{\circ} \mathrm{N}\right.$ $\left.102.132^{\circ} \mathrm{E}\right)$, Sayan, 11.VII.1965, Gorodkov (9 $\Im^{7} \sigma^{7}, 7$ of, ZISP); Chukotka: bank of the River Anadyr $\left(64.72^{\circ} \mathrm{N} 175.21^{\circ} \mathrm{E}\right), 25 . \mathrm{VI}$.19.VII.2014, A. Barkalov, V. Zinchenko (25 $\sigma^{7} \sigma^{7}, 18$ 우, ZMUM and ISEA); the upper reaches of the River Bol'shaya $\left(63.018^{\circ} \mathrm{N}\right.$ $\left.171.846^{\circ} \mathrm{E}\right), 16$ and 18.VII.1959, Gorodkov (2 $9 \circ$, ZISP); Irkutsk Oblast: Nizhneudinsk $\left(54.904^{\circ} \mathrm{N} 99.038^{\circ} \mathrm{E}\right)$, VII.1912, Parchevskaya ( 1 , ZISP); same place, 16 and 17.VI.1917, Matusevich ( $1 \sigma^{7}, 3$ 우, ZISP); Listvenichnoe [=Listvyanka] $\left(51.863^{\circ} \mathrm{N} 104.866^{\circ} \mathrm{E}\right)$, 16.VII.1938, Dombrovskaya (2 OO, ZISP); same place, 21.VI.1965, Negrobov (2 $\sigma^{7} \sigma^{7}$, ZISP); Kamchatka Krai: Kozyrevsk $\left(56.048^{\circ} \mathrm{N}\right.$ $\left.159.870^{\circ} \mathrm{E}\right)$, 14.VII.1985, V. Zlobin (3 우, ZISP); PetropavlovskKamchatskiy $\left(53.013^{\circ} \mathrm{N} 158.657^{\circ} \mathrm{E}\right), 25 . \mathrm{VI} .1959$, Gorodkov $\left(2 \bigcirc^{\top} \sigma^{7}\right.$, 2 우, ZISP); Karelia: Primorskiy $\left(66.546^{\circ} \mathrm{N} 33.103^{\circ} \mathrm{E}\right), 5$ and 6.VII.2010, A.L. Ozerov (1 $\sigma^{7}, 1$ \%, ZMUM); Poyakonda $\left(66.589^{\circ} \mathrm{N}\right.$ 33.821 ${ }^{\circ}$ E), 7.VII.2010, A.L. Ozerov (1 ${ }^{7}$, ZMUM); Komi: Seida $\left(67.05^{\circ} \mathrm{N} 63.09^{\circ} \mathrm{E}\right), 23$. VII.2010, N. Vikhrev (1 $\sigma^{7}$, ZMUM); UstTsilma $\left(65.440^{\circ} \mathrm{N} 52.153^{\circ} \mathrm{E}\right)$, 9.VIII.1978, Gorodkov (1 $\sigma^{7}$, ZISP); Ust'-Usa $\left(65.980^{\circ} \mathrm{N} 56.911^{\circ} \mathrm{E}\right), 13$.VIII.1978, Gorodkov (1 ㅇ, ZISP); Krasnoyarsk Krai: Kryuchkovo station $\left(56.096^{\circ} \mathrm{N} 62.109^{\circ} \mathrm{E}\right), 13-$ 15.VII.2009, K. Tomkovich (1 9 , ZMUM); Tanzybei $\left(53.03^{\circ} \mathrm{N}\right.$ 93.18 ${ }^{\circ}$ ), 26.VI.2017, N. Vikhrev (1 $\sigma^{7}$, ZMUM); Leningrad Oblast: Rozhdestveno $\left(59.322^{\circ} \mathrm{N} 29.946^{\circ} \mathrm{E}\right), 4,15$ and 28.VI.1956, A Stackelberg ( $2 \sigma^{\top}, 1$, ZISP); Moscow and Moscow Oblast: Molzhaninovka $\left(55.936^{\circ} \mathrm{N} 37.385^{\circ} \mathrm{E}\right), 13 . V .2010$, A.L. Ozerov (2 $\sigma^{\top} \sigma^{\top}$, ZMUM); Izmaylovo $\left(55.786^{\circ} \mathrm{N} 37.835^{\circ} \mathrm{E}\right)$, 5.VI.1983, A.L. Ozerov (1 + , ZMUM); $35 \mathrm{~km} \mathrm{NNE}$ of Moscow $\left(56.200^{\circ} \mathrm{N} 37.833^{\circ} \mathrm{E}\right)$,
26.VI.1993, A.L. Ozerov (4 $\sigma^{7} \sigma^{\nearrow}, 1$ ค, ZMUM); Snegiri $\left(55.887^{\circ} \mathrm{N}\right.$ $37.028^{\circ}$ E), 26.V.1984, Gorodkov (1 $\circ$, ZISP); Primorsky Krai: Spassk $\left(44.600^{\circ} \mathrm{N} 132.816^{\circ} \mathrm{E}\right), 6$. VI.1961, Zhelokhovtsev (1 +, ZMUM); Andreevka env. (42.7 $\left.{ }^{\circ} \mathrm{N} 131.1^{\circ} \mathrm{E}\right), 26-31 . V I I .2018, \mathrm{~N}$. Vikhrev (1 ${ }^{7}, 3$ $\left.{ }^{\circ}, \mathrm{ZMUM}\right)$; Sverdlov Oblast: Uktus station $\left(56.780^{\circ} \mathrm{N} 60.628^{\circ} \mathrm{E}\right)$, 9.VII.1910, G. Yakobson ( 1 , ZISP); Tyumen' Oblast: $\left(63.766^{\circ} \mathrm{N}\right.$

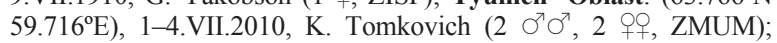
River It'yakh $\left(61.85^{\circ} \mathrm{N} 69.06^{\circ} \mathrm{E}\right), 22$.VII.2010, K. Tomkovich $(1$, ZMUM); Saranpaul' $\left(64.257^{\circ} \mathrm{N} 60.917^{\circ} \mathrm{E}\right), 23$. VI.1989, Malozemov (1 O, ZISP); Obdorsk [now=Salekhard] $\left(66.53^{\circ} \mathrm{N} 66.613^{\circ} \mathrm{E}\right)$, 14.VIII.1925, Fridolin (1 $\sigma^{7}$, ZISP); Tuva: River Uyuk $\left(52.07^{\circ} \mathrm{N}\right.$ $\left.94.04^{\circ} \mathrm{E}\right), 800 \mathrm{~m}, 1-3$. VII.2017, N. Vikhrev (2 $\mathrm{O}^{7} \mathrm{\sigma}^{7}$, ZMUM); Yakutia: $20 \mathrm{~km}$ above of the mouth of the River Biryuk $\left(60.434^{\circ} \mathrm{N}\right.$

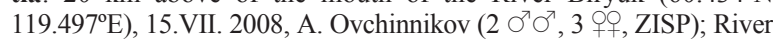
Biryuk, $4 \mathrm{~km}$ below of the mouth of the River Melichan (ca. $60.434^{\circ} \mathrm{N}$ $\left.119.495^{\circ} \mathrm{E}\right), 13$. VII.2008, A. Ovchinnikov (1 $\circ$, ZISP); River Yana, Stolby env. $\left(67.531^{\circ} \mathrm{N} 134.087^{\circ} \mathrm{E}\right), 26-28 . V I I .2008$, A. Ovchinnikov (2 $\sigma^{7} \sigma^{7}, 7$ 우, ZISP); Yakutsk env., Chochur-Muran $\left(62.034^{\circ} \mathrm{N}\right.$ $\left.129.618^{\circ} \mathrm{E}\right), 15$.VIII.1972, Gorodkov (1 9 , ZISP); Khaptagay $\left(61.787^{\circ} \mathrm{N}\right.$ 129.795 ${ }^{\circ}$ ), 7.VI.1974, Bagachanova (1 $\sigma^{7}$, ZISP); Balagannakh $\left(64.495^{\circ} \mathrm{N} 143.817^{\circ} \mathrm{E}\right), 4$ and 7.VII.1974, Nartshuk (2 $\sigma^{7} \sigma^{\top}, 1$, ZISP); River Yana opposite of Verkhoyansk $\left(67.550^{\circ} \mathrm{N} 133.359^{\circ} \mathrm{E}\right)$, 23.VII.1974, Nartshuk (1 $\sigma^{7}, 1$ ㅇ, ZISP); Olekminsk env. $\left(60.370^{\circ} \mathrm{N}\right.$ $\left.120.436^{\circ} \mathrm{E}\right), 2$.VIII.1974, Nartshuk (1 9 , ZISP); Verkhoyansk $\left(67.548^{\circ} \mathrm{N}\right.$ $\left.133.396^{\circ} \mathrm{E}\right), 23$. VII.1974, Gorodkov (1 + , ZISP); the mouth of the River Vityuy-ust, River Bakhanay $\left(66.014^{\circ} \mathrm{N} 123.654^{\circ} \mathrm{E}\right), 17 . \mathrm{VI} .1875$, Chekanovskiy ( 1 , ZISP); Zabaykalsky Krai: Ulyatuy $\left(51.172^{\circ} \mathrm{N}\right.$

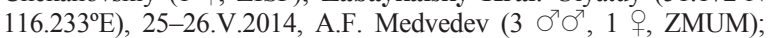
Bylyra $\left(49.689^{\circ} \mathrm{N} 111.720^{\circ} \mathrm{E}\right), 19 . \mathrm{VI} .1975$, V. Richter (1 Oे, ZISP).

ADDITIONAL MATERIAL EXAMINED. Mongolia: Songino (47.851ำ $\left.106.664^{\circ} \mathrm{E}\right), 3-4 . I X .1969$, Zaitzev (1 ○, ZISP).

DIAGNOSTIC DESCRIPTION. Male. Female. Body-length 4.7-8.0 mm. Head. Frontal vitta from reddish yellow to blackish, with whitish reflection; frontoorbital plate black, greyish dusted. Face and parafacial yellow with whitish reflection. Gena from yellow to blackish. Postcranium black, greyish dusted. 3 orbital and 2-3 frontal setae present. Antenna black. Postpedicel approximately 2 times as long as wide. Arista plumose, the longest hairs approximately equal to the width of postpedicel. Palpus black. Thorax black, greyish dusted. Acrostichals absent, dorsocentrals $3+3$ (including scapular seta), intra-alars $(0-1)+1$, supra-alars $1+2,2$ postpronotals. Scutellum with a pair of strong discal and a pair of strong apical setae. Legs delicately greyish dusted. Femora of all legs black; fore tibia and tarsi of all legs yellow; mid and hind tibiae from yellow to blackish. Wing tinged with brownish; veins brownish. Vein $R_{1}$ bare on apical third of dorsal surface. Abdomen black, greyish dusted. Male sternite 4 rectangular, wider than long. Male sternite 5, epandrium and surstyli as in Figs 24-26.

DISTRIBUTION. Russia: Altai, Amur Oblast, Arkhangelsk Oblast, Bashkiria, Buryatia, Chukotka, Irkutsk Oblast, Kamchatka Krai, Karelia, Khabarovsk Krai, Komi, Leningrad Oblast, Magadan Oblast, Moscow Oblast, Murmansk Oblast, Primorsky Krai, Sverdlov Oblast, Tyumen' Oblast, Tuva, Yakutia, Zabaykalsky Krai. — Europe, Mongolia [Gorodkov, 1986: 14; Šifner, 2008: 117].

\section{Cordilura aemula (Collin, 1958)} Figs 4, 27-29.

aemula Collin, 1958: 43 (Cordylura). Type-localities: "Barton Mills (Suffolk)" ... "Horning Ferry (Norfolk)" (Great Britain). 

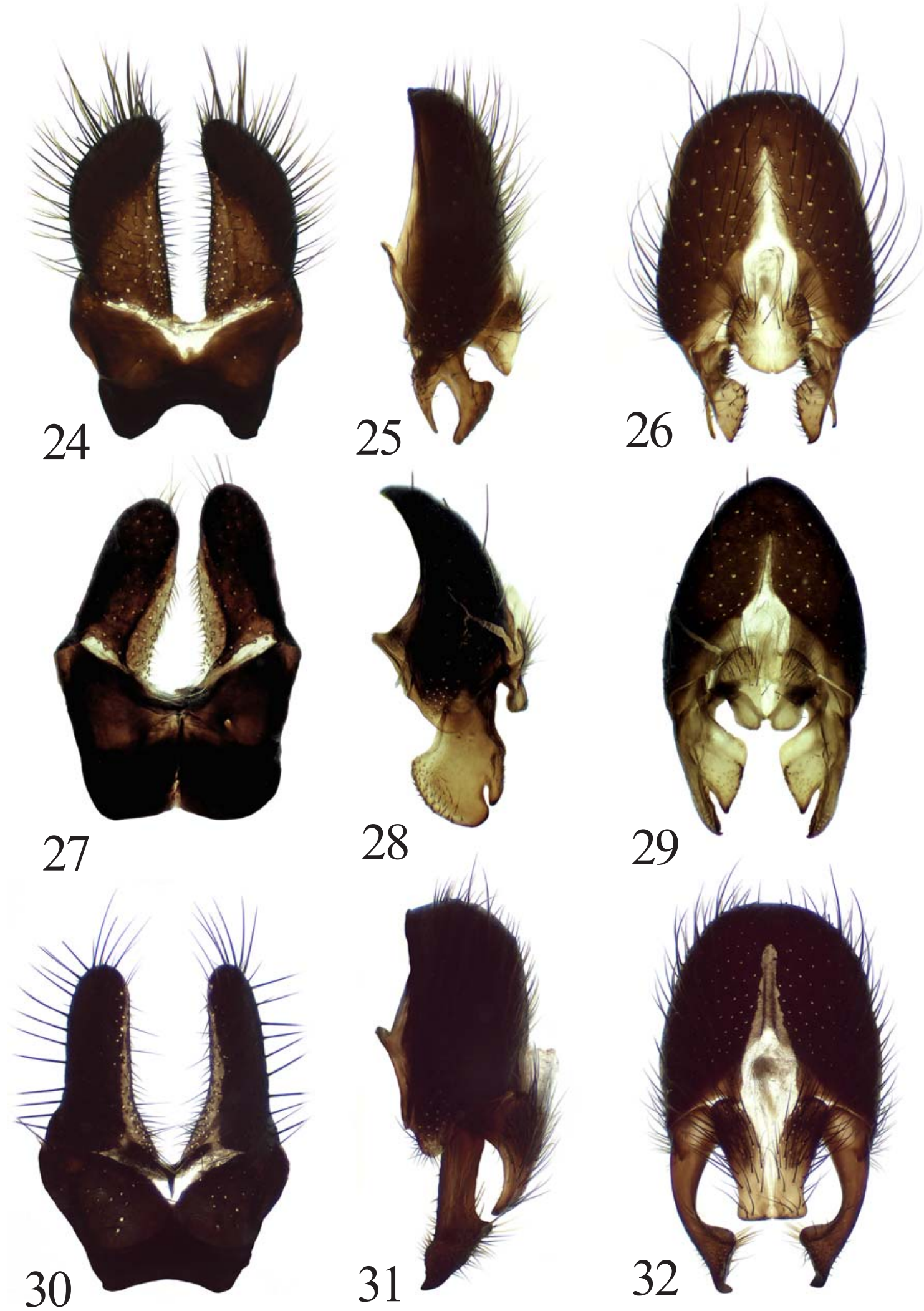

Figs 24-32. Cordilura aberrans (Becker) (24-26), C. aemula (Collin) (27-29) and C. albilabris (Fabricius) (30-32): 24, 27, $30-$ male sternite 5; 25, 28, 31 - epandrium and surstyli, lateral view; 26, 29, 32 - same, dorsal view.

Рис. 24-32. Cordilura aberrans (Becker) (24-26), C. aemula (Collin) (27-29) и C. albilabris (Fabricius) (30-32): 24, 27, 30 стернит 5 самца; 25, 28, 31 - эпандрий и сурстили, сбоку; 26, 29, 32 - то же, сверху. 
REMARK. This species was recorded in Russia from Far East (including Amur Oblast and Khabarovsk Krai) by Ozerov \& Krivosheina [2014: 206].

MATERIAL EXAMINED. Kamchatka Krai: Uzon $\left(54.503^{\circ} \mathrm{N}\right.$ $159.99^{\circ}$ E), 20.VII.2009, L. Lobkova (1 + , ZMUM); Primorsky Krai: Kedrovaya Pad' Nature Reserve $\left(43.104^{\circ} \mathrm{N} 131.512^{\circ} \mathrm{E}\right), 8_{-}$ 16.VI.2007, A. Ovchinnikov (1 $0^{7}$, ZISP); $15 \mathrm{~km} \mathrm{SW}$ of Slavyanka (ca. $\left.42.824^{\circ} \mathrm{N} 131.234^{\circ} \mathrm{E}\right), 16 . \mathrm{VI} .1993$, Belokobyl'skiy (1 $\circ$, ZISP); Sikhote-Alin Nature Reserve, Blagodatnoe $\left(44.975^{\circ} \mathrm{N} 136.526^{\circ} \mathrm{E}\right)$, 1-6.VI.2018, M. Sergeev (2 $\sigma^{7} \sigma^{7}$, ZMUM); Yakutia: Yakutsk env. $\left(62.159^{\circ} \mathrm{N} 129.822^{\circ} \mathrm{E}\right), 24 . \mathrm{VI} .1957$, Gorodkov (1 $0^{7}$, ZISP).

DIAGNOSTIC DESCRIPTION. Male. Female. Body-length 7.9-9.2 mm. Head. Frontal vitta reddish yellow, with whitish reflection; fronto-orbital plate black, greyish dusted. Face and parafacial yellow with whitish reflection. Gena yellow or brownish. Postcranium black, grey dusted. 3 orbital and 2-4 frontal setae present. Antenna black. Postpedicel approximately 2-2.5 times as long as wide. Arista pubescent, the longest hairs approximately equal to $1 / 3-1 / 2$ width of postpedicel (Fig. 4). Palpus black or brown. Thorax black, greyish dusted. Acrostichals setulose in two rows, dorsocentrals $3+3$ (including small scapular seta), intra-alars $1+2$, supra-alars $1+2,2$ postpronotals. Scutellum with a pair of strong discal and a pair of strong apical setae. Legs greyish dusted. Femora of all legs black; tibiae and tarsi of all legs yellow; tarsomeres 2-4 of fore leg each with small black spot basally. Wing tinged with yellowish; veins yellowish. Vein $\mathrm{R}_{1}$ setulose on apical third of dorsal surface. Abdomen black, greyish dusted. Male sternite 4 rectangular, longer than wide. Male sternite 5, epandrium and surstyli as in Figs 27-29.

DISTRIBUTION. Russia: Amur Oblast, Kamchatka Krai, Khabarovsk Krai, Primorsky Krai, Yakutia. - Europe.

\section{Cordilura albilabris (Fabricius, 1805)}

Figs 7, 30-32.

albilabris Fabricius, 1805: 315 (Ocyptera). Type-locality: "Habitat in Austria".

scirpi Robineau-Desvoidy, 1830: 669 (Phrosia). Type-locality: not given ("assez commune") (?France).

REMARK. Noted by Gorodkov [1986: 37] for European part of Russia without indicating specific locality.

MATERIAL EXAMINED. Adygea: Dakhovskaya env. $\left(44.199^{\circ} \mathrm{N} 40.170^{\circ} \mathrm{E}\right), 465 \mathrm{~m}, 18-31$. VIII.2009, K. Tomkovich (3 $\sigma^{7} \sigma^{\top}, 1$ ㅇ, ZMUM); Maykop (44.614º $\left.40.106^{\circ} \mathrm{E}\right), 30 . \mathrm{VI} .1911$, G. Shaposhnikov (1 $\sigma^{7}, 1$, ZISP); Astrakhan' Oblast: Zyuzino $\left(45.751^{\circ} \mathrm{N} 47.678^{\circ} \mathrm{E}\right), 8-9 . V .2010$, K. Tomkovich (1 $\left.0^{7}, \mathrm{ZMUM}\right)$ Chechenya: Tazbichi ravine, $6 \mathrm{~km} \mathrm{~S}$ of Itum-Kale $\left(42.708^{\circ} \mathrm{N}\right.$ $\left.45.598^{\circ} \mathrm{E}\right), 8 . \mathrm{VI}_{1} 1972$, V. Richter (1 $\sigma^{\top}$, ZISP); Crimea: $\left(45.505^{\circ} \mathrm{N}\right.$ $\left.34.135^{\circ} \mathrm{E}\right), 27$. VIII.1926, Zimin (1 ㅇ, ZISP); Simferopol' env. $\left(44.951^{\circ} \mathrm{N} 34.115^{\circ} \mathrm{E}\right), 10 . \mathrm{VI} .1911$, Pavlovskiy (1 $\sigma^{7}$, ZISP); Kaliningrad Oblast: Primorsk $\left(54.726^{\circ} \mathrm{N} 20.004^{\circ} \mathrm{E}\right), 19-21$. VIII.2013, K.Tomkovich (1 $\sigma^{7}$, ZMUM); Krasnodar Krai: L'vovskoe $\left(44.992^{\circ} \mathrm{N} 38.631^{\circ} \mathrm{E}\right), 25 . \mathrm{VII} .1956,27 . \mathrm{IV}$. and 9.V.1957, G

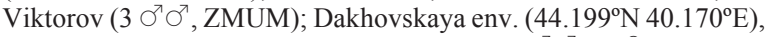

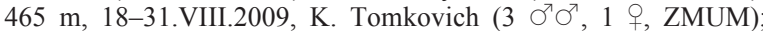
Mezmayka $\left(44.197^{\circ} \mathrm{N} 39.957^{\circ} \mathrm{E}\right), 27 . \mathrm{VIII} .2005$, A. Ovchinnikov (2 $\sigma^{7} \sigma^{7}$, ZISP); Teberdinsky Nature Reserve, Dzhamagat Valley $\left(43.464^{\circ} \mathrm{N} 41.736^{\circ} \mathrm{E}\right), 8$.VII.1982, Nartshuk (1 $\sigma^{7}$, ZISP); Kursk Oblast: Oboyan' $\left(51.191^{\circ} \mathrm{N} 36.312^{\circ} \mathrm{E}\right), 26$. V.2007, N. Vikhrev (2 $\sigma^{7} \sigma^{7}, 1$. ZMUM); same place, 25-26.V. and 20-21.VII.2007, A.L. Ozerov (5 $\sigma^{\top} \bigcirc^{\top}, 3$ 우, ZMUM); Leningrad Oblast: Yashchera $\left(58.894^{\circ} \mathrm{N} 29.820^{\circ} \mathrm{E}\right), 26 . V .1957$, A. Stackelberg (3 $\sigma^{7} \sigma^{7}, 2$ 우, ZISP); Mordovia: Mordovsky Nature Reserve, River Pushta (54.71 $\left.{ }^{\circ} \mathrm{N}, 43.22^{\circ} \mathrm{E}\right), 8-12 . \mathrm{VI} .2020$, N. Vikhrev (1 $\sigma^{7}$, ZMUM); Moscow and Moscow Oblast: Bittsa $\left(55.641^{\circ} \mathrm{N} 37.570^{\circ} \mathrm{E}\right)$, 26.VII.1936, B. Rohdendorf (1 $\sigma^{7}, 1$ q, ZMUM); Izmaylovo $\left(55.803^{\circ} \mathrm{N} 37.841^{\circ} \mathrm{E}, 55.802^{\circ} \mathrm{N} 37.844^{\circ} \mathrm{E}\right), 20 . \mathrm{VI} .2007,17 . \mathrm{V} .2008$,

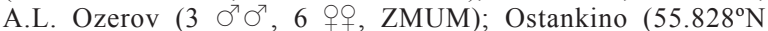
$\left.37.612^{\circ} \mathrm{E}\right), 29$. VII.1935 (1 $\mathrm{\sigma}^{7}$, ZMUM); Strogino $\left(55.793^{\circ} \mathrm{N}\right.$ $\left.37.408^{\circ} \mathrm{E}\right), 19$. V.20011, N. Vikhrev (1 $\sigma^{\top}$, ZMUM); Troitse-Lykovo $\left(55.794^{\circ} \mathrm{N} 37.418^{\circ} \mathrm{E}\right), 21$. VI.2009, N. Vikhrev (1 $\sigma^{\top}$, ZMUM); Abramtsevo $\left(56.230^{\circ} \mathrm{N} 37.956^{\circ} \mathrm{E}\right), 12 . \mathrm{VIII} .1957$, E.S. Smirnov (2 $\mathrm{O}^{7} \sigma^{7}, 5$ 우, ZMUM); Golitsyno $\left(55.649^{\circ} \mathrm{N} 37.011^{\circ} \mathrm{E}\right), 21 . \mathrm{V} .1977$, 9.VII.1983, A. Shatalkin (1 ơ, 1 \%, ZMUM); Dmitrov env. $\left(56.316^{\circ} \mathrm{N} 37.725^{\circ} \mathrm{E}\right), 11$. VII.2007, 9.VI.2009, N. Vikhrev (2 プ フ', ZMUM); Mamontovka $\left(55.999^{\circ} \mathrm{N} 37.816^{\circ} \mathrm{E}\right), 3$ and 16.VII.1956, E. Smirnov (2 $\left.\sigma^{7} \sigma^{\top}, \mathrm{ZMUM}\right)$; Stepan'kovo $\left(55.004^{\circ} \mathrm{N} 35.624^{\circ} \mathrm{E}\right)$, 15.VII.2006, 3.VI.2007, A.L. Ozerov (3 $\sigma^{7} \sigma^{7}, 4$ क्ष, ZMUM); Ivanovskoe $\left(55.933^{\circ} \mathrm{N} 35.627^{\circ} \mathrm{E}\right), 14 . \mathrm{V} .2006$, A.L. Ozerov (1 O, ZMUM); Smolevo $\left(55.626^{\circ} \mathrm{N} 38.964^{\circ} \mathrm{E}\right), 2005$, K. Tomkovich (1 9, ZMUM); Yakshino $\left(55.922^{\circ} \mathrm{N} 35.577^{\circ} \mathrm{E}\right), 17$. VII.2008, A.L. Ozerov (1 $\left.\sigma^{7}, \mathrm{ZMUM}\right)$; Dubna env. $\left(56.748^{\circ} \mathrm{N} 37.24^{\circ} \mathrm{E}\right)$, 17.VIII.20015, N. Vikhrev (1 $\Im^{7}$, ZMUM); Orenburg Oblast: 10 $\mathrm{km}$ E of Tashla $\left(51.771^{\circ} \mathrm{N} 52.860^{\circ} \mathrm{E}\right), 6-8 . V I I I .2004$, Nartshuk (1 $\sigma^{\top}, 3$ 우, ZISP); Tyumen' Oblast: Tyumen' Town $\left(57.15^{\circ} \mathrm{N}\right.$ $\left.65.53^{\circ} \mathrm{E}\right), 13$.VII.1964, Veselkin (1 9 , ZISP); Volgograd Oblast: Torgun env. $\left(50.300^{\circ} \mathrm{N} 47.209^{\circ} \mathrm{E}\right), 13 . \mathrm{VII} .2012$, Astakhov (1 $\sigma^{\top}, 1$ †, ZISP); Verkhniy Balykley env. (49.535 $\left.\mathrm{N} 45.177^{\circ} \mathrm{E}\right)$, 9.VIII.2012, Astakhov (1 $\sigma^{7}$, ZISP).

ADDITIONAL MATERIAL EXAMINED. Armenia: Kirovakan [=Vanadzor] $\left(40.807^{\circ} \mathrm{N} 44.493^{\circ} \mathrm{E}\right), 11$. VII.1995, Zagulyaev (1 9 , ZISP); Kazakhstan: River Bol'soy Bukon', 25 km ENE of Kokpekty, Semipalatinsk Oblast, 2.VIII.1978, Nartshuk (1 $\sigma^{\top}$, ZISP); Kokshetau $\left(53.285^{\circ} \mathrm{N} 69.396^{\circ} \mathrm{E}\right), 6$.VIII.1957, Nartshuk (1 $\left.{ }^{\prime}, \mathrm{ZISP}\right)$; Kirgyzstan: Ak-Terek $\left(41.293^{\circ} \mathrm{N} 72.823^{\circ} \mathrm{E}\right), 1800 \mathrm{~m}, 12 \mathrm{~km}$ W of Arslanbob, 18-22.VIII.1986, Tanasiychuk (1 O', ZISP); Rybach'e [=Balykchi] $\left(42.465^{\circ} \mathrm{N} 76.182^{\circ} \mathrm{E}\right), 1600 \mathrm{~m}, 5 . \mathrm{VIII} .1969$, Nartshuk (1 $\overbrace{}^{7}$, ZISP).

DIAGNOSTIC DESCRIPTION. Male. Female. Body-length 5.2-7.2 mm. Head (Fig. 7). Frontal vitta from yellow to black; fronto-orbital plate black, shining. Face yellowish, with whitish reflection. Parafacial and gena from yellow to black. Postcranium black completely, mostly shining. 2 orbital and 4 frontal setae present. Scapus and pedicel brown; postpedicel blackish, approximately 3-3.5 times as long as wide (Fig. 7). Arista pubescent, the longest hairs approximately equal to $1 / 3-$ 1/2 width of postpedicel. Palpus yellow. Thorax black; scutum shining with greyish stripe in centre, anepisternum and anepimeron mostly shining. Acrostichals absent, dorsocentrals 3+3 (including scapular seta), intraalars absent, supra-alars $0+2,1$ postpronotal. Scutellum with a pair of strong discal setae only; apical scutellar setae as setulae or absent. Legs shining, yellow, only hind tibia and tarsi of all legs brownish. Wing tinged with brownish; veins blackish. Vein $\mathrm{R}_{1}$ setulose on apical third of dorsal surface. Abdomen black, shining or subshining. Male sternite 4 rectangular, longer than wide. Male sternite 5, epandrium and surstyli as in Figs 30-32.

Description and figures of female ovipositor are given by Ovchinnikov [2009: 624] (as Phrosia albilabris).

DISTRIBUTION. Adygea, Astrakhan' Oblast, Chechenya, Crimea, Kaliningrad Oblast, Krasnodar Krai, Kursk Oblast, Leningrad Oblast, Mordovia, Moscow Oblast, Orenburg Oblast, Tyumen' Oblast, Volgograd Oblast. — Europe, Azerbaijan [Šifner, 2008: 125]; Armenia (first record), Kazakhstan (first record), Kyrgyzstan (first record). 
Cordilura albipes (Fallén, 1819)

Figs 12, 21-23, 33-35.

albipes Fallén, 1819: 9 (Cordylura). Type-locality: "in Quasa Esperöd et in silva Gyllebo Scaniae" (Scåne, Sweden).

bilineata Meigen, 1838: 340 (Cordylura). Type-locality: not given ("Hiesige Gegend", ?nr Aachen).

unicolor Loew, 1864: 17 (Cordylura). Type-locality: "Andalusien" (Spain).

REMARK. This species was recorded in Russia from European part and from Siberia without indicating specific locality by Gorodkov [1970: 446, 1986: 16]; noted from Karelia [Humala, Polevoi, 2009: 71], Far East [Ozerov, 2009: 379; Ozerov, Krivosheina, 2014: 206] and Yakutia [Bagachanova et al., 2016: 779].

MATERIAL EXAMINED. Adygea: Lagonaki $\left(44.093^{\circ} \mathrm{N}\right.$ $\left.40.019^{\circ} \mathrm{E}\right), 1725 \mathrm{~m}, 26-28 . \mathrm{VI} .2009$, K. Tomkovich $\left(5 \sigma^{7} \sigma^{7}\right.$, ZMUM); Dakhovskaya env. (44.201 $\left.{ }^{\circ} \mathrm{N} 40.149^{\circ} \mathrm{E}\right), 12-13$. VII.2012, K. Tomkovich (1 $\sigma^{7}$, ZMUM); Altai: Bol'shoy Yaloman $\left(50.489^{\circ} \mathrm{N}\right.$ 86.416 ${ }^{\circ}$ E), 7-9.VII.1967, R. Kamenskaya ( $\sigma^{7} \sigma^{7}, 4$ +o , ZMUM); Seminsky Pass, $\left(51.06^{\circ} \mathrm{N} 85.59^{\circ} \mathrm{E}\right), 1650 \mathrm{~m}, 27-30 . \mathrm{VI} .2016, \mathrm{~N}$ Vikhrev (1 $\sigma^{7}$, ZMUM); Arkhangelsk Oblast: Vas'kovo $\left(64.413^{\circ} \mathrm{N}\right.$ 40.457E), 18.VII.1996, Gorodkov (1 $\sigma^{7}$, ZISP); Buryatia: Barguzinsky Nature Reserve, Sosnovka env. (ca. $54.182^{\circ} \mathrm{N} 109.539^{\circ} \mathrm{E}$ ), 4.VIII.1962, Gorodkov (1 $\sigma^{7}$, ZISP); Chelyabinsk Oblast: Taganay $\left(55.277^{\circ} \mathrm{N} 59.795^{\circ} \mathrm{E}\right), 18-24$.VII.2008, K. Tomkovich $\left(4 \sigma^{7} \sigma^{7}\right.$ 1 , ZMUM); Crimea: Alushta-Rybachie (44.7-8 $\left.8^{\circ} 34.4-6^{\circ} \mathrm{E}\right)$, 18-25.IV.2014, N. Vikhrev (2 $\sigma^{7} \sigma^{7}, 1$ \%, ZMUM); Dagestan: 15 km SW of Sergocala (ca. $42.462^{\circ} \mathrm{N} 47.662^{\circ} \mathrm{E}$ ), 15.VII.1983, Nartshuk (1 $\sigma^{\top}$, ZISP); Irkutsk Oblast: Baykal Port $\left(51.874^{\circ} \mathrm{N}\right.$ $\left.104.792^{\circ} \mathrm{E}\right), 25$. VI.1965, Negrobov (1 9 , ZISP); Listvyanka $\left(51.863^{\circ} \mathrm{N} 104.866^{\circ} \mathrm{E}\right), 21 . \mathrm{VI} .1965$, Negrobov (1 , , ZISP); Karelia: Primorskiy $\left(66.546^{\circ} \mathrm{N} 33.103^{\circ} \mathrm{E}\right), 1-6 . V I I .2010$, A.L. Ozerov ( $1 \mathrm{O}^{\mathrm{T}}, 10$ 우, ZMUM); Poyakonda $\left(66.589^{\circ} \mathrm{N} 33.821^{\circ} \mathrm{E}\right), 7 . \mathrm{VII} .2010$ A.L. Ozerov (1 $\sigma^{7}$, ZMUM); Kivach Nature Reserve (ca. $62.295^{\circ} \mathrm{N}$ $\left.33.921^{\circ} \mathrm{E}\right), 21 . V I .1979$, Gorodkov (3 $\sigma^{\top} \sigma^{\top}, 2$ 우, ZISP); Kemerovo Oblast: Tisul' $\left(55.759^{\circ} \mathrm{N} 88.310^{\circ} \mathrm{E}\right), 21 . \mathrm{VI} .1911$, Gorchakovskiy (2 $\sigma^{7} \sigma^{7}$, ZISP); Krasnodar Krai: Azish-Tau ridge, "Kamyshanova Polyana" (44.168 N, $\left.40.044^{\circ} \mathrm{E}\right), 1240 \mathrm{~m}, 10 . \mathrm{IV} .-3 . V .2010$, Kustov (1 ऽ', 2 Oo, ZMUM); Sochi, Mt. Akhun $\left(43.523^{\circ} \mathrm{N} 39.879^{\circ} \mathrm{E}\right)$ 25.IV.2008, N. Vikhrev (1 $\sigma^{7}$, ZMUM); Sochi, Estosadok env., Mt. Psekhako $\left(43.691^{\circ} \mathrm{N} 40.366^{\circ} \mathrm{E}\right), 14-18 . V I .2008, \mathrm{~K}$. Tomkovich

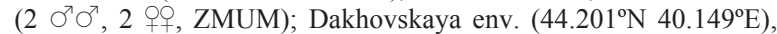
12-13.VII.2012, K. Tomkovich (1 $\sigma^{7}$, ZMUM); Lagonaki $\left(44.093^{\circ} \mathrm{N}\right.$ $\left.40.019^{\circ} \mathrm{E}\right), 1725 \mathrm{~m}, 26-28 . V I .2009, \mathrm{~K}$. Tomkovich $\left(5 \sigma^{7} \sigma^{7}\right.$, ZMUM); Teberdinsky Nature Reserve (ca. $\left.43.443^{\circ} \mathrm{N} 41.738^{\circ} \mathrm{E}\right), 15$ and 20.V.1964, Gorodkov ( $3 \sigma^{7} \sigma^{\top}, 3$ 우, ZISP); Teberdinsky Nature Reserve (ca. $43.405^{\circ} \mathrm{N} 41.721^{\circ} \mathrm{E}$ ), 3-19.V.1964, Gorodkov (2 $\sigma^{\top} \sigma^{\top}, 2$ ㅇ, ZISP); Krasnoyarsk Krai: Kryuchkovo station $\left(56.096^{\circ} \mathrm{N} 92.109^{\circ} \mathrm{E}\right), 14-23$.VII.2009, K. Tomkovich (5 ช $\sigma^{7}, 5$ 오, ZMUM); Ergaki Nature Park $\left(52.84^{\circ} \mathrm{N} 93.25^{\circ} \mathrm{E}\right), 1450 \mathrm{~m}, 27$ 29.VI.2017, N. Vikhrev (3 $\sigma^{\top} \sigma^{\top}$, ZMUM); Leningrad Oblast: Luga (ca. $58.73^{\circ} \mathrm{N} 29.84^{\circ} \mathrm{E}$ ), 21.VI. and 8.VIII.1955, A. Stackelberg (2 $\sigma^{7} \sigma^{7}$, ZISP); Rozhdestveno $\left(59.322^{\circ} \mathrm{N} 29.946^{\circ} \mathrm{E}\right)$ 15.VI.1957, A. Stackelberg (2 90 , ZISP); Sablino (ca. $59.63^{\circ} \mathrm{N}$ $\left.30.76^{\circ} \mathrm{E}\right)$, 16.VI.1923, A. Stackelberg (1 $\sigma^{7}$, ZISP); Mordovia: Mordovsky Nature Reserve, Cordon Novin'kovsky $\left(54.931^{\circ} \mathrm{N}\right.$, 43.42 $\left.1^{\circ} \mathrm{E}\right), 4-7 . V I I .2020$, K. Tomkovich (1 $\sigma^{7}$, ZMUM); Moscow and Moscow Oblast: Bittsa $\left(55.641^{\circ} \mathrm{N} 37.570^{\circ} \mathrm{E}\right), 24$.VI., 2631.VII.1936, B. Rohdendorf (1 ○, 7 우, ZMUM); same place,

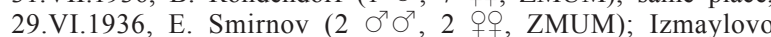
$\left(55.800^{\circ} \mathrm{N} 37.832^{\circ} \mathrm{E}, 55.802^{\circ} \mathrm{N} 37.840^{\circ} \mathrm{E}\right)$, 5.IX.1981, 26-30.V. and 5.VI.1982, 17.IV, 26.V. and 2.VII.1983, 18.V.2008, A.L. Ozerov $\left(9 \sigma^{7} \sigma^{\top}, 5\right.$ oo. ZMUM); Kuntsevo $\left(55.720^{\circ} \mathrm{N} 37.473^{\circ} \mathrm{E}\right), 17,19$ and 26.V., 18.VI. and 2.VII.1983, 13.V.1992, 16.VI.1993, A.L. Ozerov (4 $\sigma^{7} \mathrm{O}^{\top}, 2$ o 0 , ZMUM); Tsaritsyno $\left(55.616^{\circ} \mathrm{N} 37.683^{\circ} \mathrm{E}\right)$, 5-10.X.2009, K. Tomkovich (1 $\sigma^{7}$, ZMUM); $20 \mathrm{~km}$ WSW of Volokolamsk $\left(55.983^{\circ} \mathrm{N} 35.616^{\circ} \mathrm{E}\right), 13$. VII.1999, A.L. Ozerov (1 $\left.\sigma^{7}, \mathrm{ZMUM}\right) ; 35 \mathrm{~km}$ NNE of Moscow $\left(56.200^{\circ} \mathrm{N} 37.833^{\circ} \mathrm{E}\right)$ 26.VI.1993, A.L. Ozerov (2 $\sigma^{7} \sigma^{7}, 1$ \%, ZMUM); Abramtsevo $\left(56.230^{\circ} \mathrm{N} 37.956^{\circ} \mathrm{E}\right), 13$.VIII.1955, E.S. Smirnov (1 9, ZMUM);
Andreevskoe $\left(55.974^{\circ} \mathrm{N} 35.604^{\circ} \mathrm{E}\right)$, 4.VIII.2005, A.L. Ozerov (2 우, ZMUM); Burtsevo env. (55.981 ${ }^{\circ} \mathrm{N} 35.597^{\circ} \mathrm{E}, 55.975^{\circ} \mathrm{N}$ $\left.35.589^{\circ} \mathrm{E}, 55.978^{\circ} \mathrm{N} 35.589^{\circ} \mathrm{E}, 55.975^{\circ} \mathrm{N} 35.586^{\circ} \mathrm{E}\right), 25 . \mathrm{V} ., 2-3$ and 18.VI.2006, 25.VII. and 1.VIII.2007, 29.V.2010, A.L. Ozerov

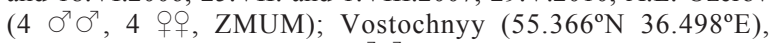
20.V.2010, D. Gavryushin (2 $\left.\sigma^{7} \sigma^{7}, \mathrm{ZMUM}\right)$; Golitsyno $\left(55.649^{\circ} \mathrm{N}\right.$ 37.011 $\left.{ }^{\circ} \mathrm{E}\right), 23 . V .1976,21 . V .1977$, 9.IX.1979, 7.VI.1980, 29.V. and 5.VI.1981, 7.VIII.1983, 3.VIII.1989, A. Shatalkin (10 $\sigma^{\top} \sigma^{\top}, 1$ +, ZMUM); Dmitrov env. $\left(56.316^{\circ} \mathrm{N} 37.725^{\circ} \mathrm{E}\right), 10 . \mathrm{VI}$. and 29.VII.2006, 19.V.2007, N. Vikhrev (4 $\sigma^{7} \sigma^{7}$, ZMUM); Zosimova Pustyn' (55.412 $\left.{ }^{\circ} \mathrm{N} 36.814^{\circ} \mathrm{E}\right), 24$ and 28.V.2010, D. Gavryushin (1 $\mathrm{O}^{7}, 1$ 9, ZMUM); Ivanovskoe $\left(55.939^{\circ} \mathrm{N} 35.621^{\circ} \mathrm{E}\right), 12$.VI.2006, A.L. Ozerov (1 $\left.\sigma^{T}, \mathrm{ZMUM}\right)$; Ignatkovo $\left(55.939^{\circ} \mathrm{N} 35.620^{\circ} \mathrm{E}\right)$, 13.VI.2008, A.L. Ozerov (1 ${ }^{\circ}$ ZMUM); Mamontovka $\left(55.999^{\circ} \mathrm{N}\right.$ $\left.37.816^{\circ} \mathrm{E}\right), 3$ and 16.VII.1956, E. Smirnov (1 $\sigma^{\top}$, ZMUM); Molzhaninovka $\left(55.936^{\circ} \mathrm{N} 37.385^{\circ} \mathrm{E}\right), 13 . \mathrm{V} .2010$, A.L. Ozerov (1 $\sigma^{7}$, ZMUM); Naro-Fominsk env. $\left(55.357^{\circ} \mathrm{N} 36.736^{\circ} \mathrm{E}, 55.364^{\circ} \mathrm{N}\right.$ $36.738^{\circ} \mathrm{E}, 55.364^{\circ} \mathrm{N} 36.741^{\circ} \mathrm{E}, 55.393^{\circ} \mathrm{N} 36.779^{\circ} \mathrm{E}, 55.375^{\circ} \mathrm{N}$ $\left.36.756^{\circ} \mathrm{E}, 55.372^{\circ} \mathrm{N} 36.757^{\circ} \mathrm{E}\right), 4$ and 16.VI.2006, 22.V., 3 and 7.VI.2007, 13.V.2008, 8.VI. and 2.VIII.2010, 18.V.2012, D. Gavryushin $\left(5 \sigma^{\top} \sigma^{7}, 5\right.$ 우, ZMUM); Ozhigovo $\left(55.453^{\circ} \mathrm{N} 36.884^{\circ} \mathrm{E}\right)$, 26.VII.2010, D. Gavryushin (1 + , ZMUM); Rodinka $\left(55.774^{\circ} \mathrm{N}\right.$ $36.291^{\circ} \mathrm{E}$ ), 5.VI.2012, D. Gavryushin (1 $\sigma^{7}$, ZMUM); Stepan'kovo $\left(55.004^{\circ} \mathrm{N} 35.624^{\circ} \mathrm{E}\right), 3 . V I .2007$, A.L. Ozerov (2 $\sigma^{7} \sigma^{7}$, ZMUM); Murmansk Oblast: Kolvitsa $\left(67.082^{\circ} \mathrm{N} 33.183^{\circ} \mathrm{E}\right)$, 8.VIII.1995, Gorodkov ( 2 +, ZISP); Nizhegorod Oblast: Dzerzhinsk $\left(56.21^{\circ} \mathrm{N}\right.$ $\left.43.62^{\circ} \mathrm{E}\right)$, 17.VIII.2009, N. Vikhrev (1 9 , ZMUM); North Ossetia - Alania: Bakhty-Laparyrag range $\left(42.938^{\circ} \mathrm{N} 44.287^{\circ} \mathrm{E}\right), 1770 \mathrm{~m}$, 1-14.VI.1989 and 28-30.VI.1990, A.L. Ozerov, A. Shatalkin (4 $\sigma^{\top} \sigma^{\top}, 6$ of, $\left.\mathrm{ZMUM}\right)$; Buron env. $\left(43.793^{\circ} \mathrm{N} 43.922^{\circ} \mathrm{E}\right), 20-$ 29.VI.1988, 6-7.VI.1989, 28.VI. and 5-6.VII.1990, A.L. Ozerov, A. Shatalkin $\left(6 \sigma^{\top} \sigma^{\top}, 7\right.$ 우, ZMUM); Alagir env. $\left(43.015^{\circ} \mathrm{N}\right.$ 44.224 $\left.{ }^{\circ} \mathrm{E}\right), 17$.VII.1990, A.L. Ozerov (1 ${ }^{\mathrm{T}}$, ZMUM); Vladikavkaz $\left(43.044^{\circ} \mathrm{N} 44.682^{\circ} \mathrm{E}\right), 16$. IV.1899, Demokidov (1 +, ZISP); Novgorod Oblast: Ramen'e $\left(58.374^{\circ} \mathrm{N} 33.430^{\circ} \mathrm{E}\right)$, Okulovka env., 4.VI.1988, Gorodkov ( $1 \sigma^{7}$, ZISP); Novosibirsk Oblast: Novosibirsk $\left(54.842^{\circ} \mathrm{N} 83.114^{\circ} \mathrm{E}\right), 14 . \mathrm{VI} .2008$, O. Kosterin (1 $\left.\sigma^{7}, \mathrm{ZMUM}\right)$; $4 \mathrm{~km} \mathrm{~S}$ of Novososedovo $\left(54.616^{\circ} \mathrm{N} 83.985^{\circ} \mathrm{E}\right), 7 . V I I .2008$, O. Kosterin (1 $\sigma^{7}, Z_{M U M}$; $\left(55.52^{\circ} \mathrm{N} 83.24^{\circ} \mathrm{E}\right), 22$.V.2011, O. Kosterin (3 $\sigma^{\top} \sigma^{\top}$, ZMUM); Primorsky Krai: Kedrovaya Pad' Nature Reserve (43.104 $\left.{ }^{\circ} \mathrm{N} 131.512^{\circ} \mathrm{E}\right), 8^{-16 . V I .2007, ~ A . ~ O v c h i n n i k o v ~(1 ~}$ , ZISP); Rostov Oblast: Kamensk-Shakhtinsky $\left(48.293^{\circ} \mathrm{N}\right.$ 40.257 E), 25.V.2011, D. Gavryushin (2 O+, ZMUM); Ryazan' Oblast: Kasimov $\left(54.94^{\circ} \mathrm{N} 41.34^{\circ} \mathrm{E}\right), 25$.VII.2013, N. Vikhrev (1 $\sigma^{7}$, ZMUM); Sakhalin Oblast: Sakhalin I., Mt. Chekhov $\left(47.005^{\circ} \mathrm{N}\right.$ $\left.142.839^{\circ} \mathrm{E}\right), 29$. VI.1973, Kasparyan (1 9 , ZISP); Tatarstan: Volzhsko-Kamsky Nature Reserve, Lake Raifa $\left(55.897^{\circ} \mathrm{N} 48.733^{\circ} \mathrm{E}\right)$, 19.VII.2007, Basov (1 $\sigma^{7}$, ZMUM); Tomsk Oblast: Basandayka $\left(56.283^{\circ} \mathrm{N} 85.489^{\circ} \mathrm{E}\right), 23$. VI.1967, Gorodkov (5 $\Im^{7} \sigma^{7}$, ZISP); Tyumen' Oblast: $\left(63.766^{\circ} \mathrm{N} 59.716^{\circ} \mathrm{E}\right), 1-4$.VII.2010, K. Tomkovich

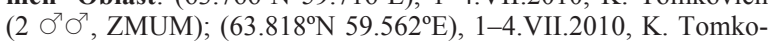
vich (3 $\sigma^{7} \sigma^{7}, 1$ \%, ZMUM); River Soromlorop'yavin $\left(62.650^{\circ} \mathrm{N}\right.$ $\left.71.891^{\circ} \mathrm{E}\right)$, 26.VII.2010, K. Tomkovich (1 +, ZMUM); Sob' env. $\left(67.07^{\circ} \mathrm{N} 65.46^{\circ} \mathrm{E}\right), 26-31 . V I I .2011, \mathrm{~K}$. Tomkovich (1 ऽ, ZMUM); Surgut $\left(61.259^{\circ} \mathrm{N} 73.418^{\circ} \mathrm{E}\right), 27 . \mathrm{VII} .1977$, Gorodkov (3 Oo, ZISP); Tuva: Kyzyl env. $\left(51.7^{\circ} \mathrm{N} 94.7^{\circ} \mathrm{E}\right), 5-7 . V I I .2017$, N. Vikhrev (1 $\sigma^{\top}$, ZMUM); Zabaykalsky Krai: Baley $\left(51.579^{\circ} \mathrm{N} 116.634^{\circ} \mathrm{E}\right)$, 18.VII.1971, V. Richter (1 ð', ZISP).

ADDITIONAL MATERIAL EXAMINED. Armenia: Bashkend $[$ now $=$ Artsvashen $]\left(40.641^{\circ} \mathrm{N} 45.523^{\circ} \mathrm{E}\right), 24$. VII.1976, Nartshuk $\left(1 \mathrm{O}^{7}\right.$, ZISP); Belarus: Belovezhskaya Pushcha $\left(52.707^{\circ} \mathrm{N} 23.862^{\circ} \mathrm{E}\right)$, 14 and 17.VII., 5.VIII.1961, Nartshuk (1 ๑ , 2 우, ZISP); Korolevo $\left(55.303^{\circ} \mathrm{N} 30.212^{\circ} \mathrm{E}\right), 27 . V I .1905$, Birulya (1 $\sigma^{7}$, ZISP); Georgia: Tsagveri $\left(41.800^{\circ} \mathrm{N} 43.481^{\circ} \mathrm{E}\right), 1200 \mathrm{~m}, 8$ and 12 .V.1969, Gorodkov (7 $\sigma^{7} \sigma^{7}, 2$ 우, ZISP); Borzhomi park $\left(41.891^{\circ} \mathrm{N} 43.321^{\circ} \mathrm{E}\right)$, 880-900 m, 16.V.1969, Gorodkov (1 o', ZISP); Mongolia: UlanBator env. (ca. $\left.47.907^{\circ} \mathrm{N} 106.931^{\circ} \mathrm{E}\right), 24-29 . V I .1970$, Nartshuk (2 $\sigma^{\top} \sigma^{\top}$, ZISP).

DIAGNOSTIC DESCRIPTION. Male. Female. Body-length 3.8-6.2 mm. Head. Frontal vitta from yellow to brownish; fronto-orbital plate yellowish. Face, parafacial and gena yellowish, with weak whitish re- 

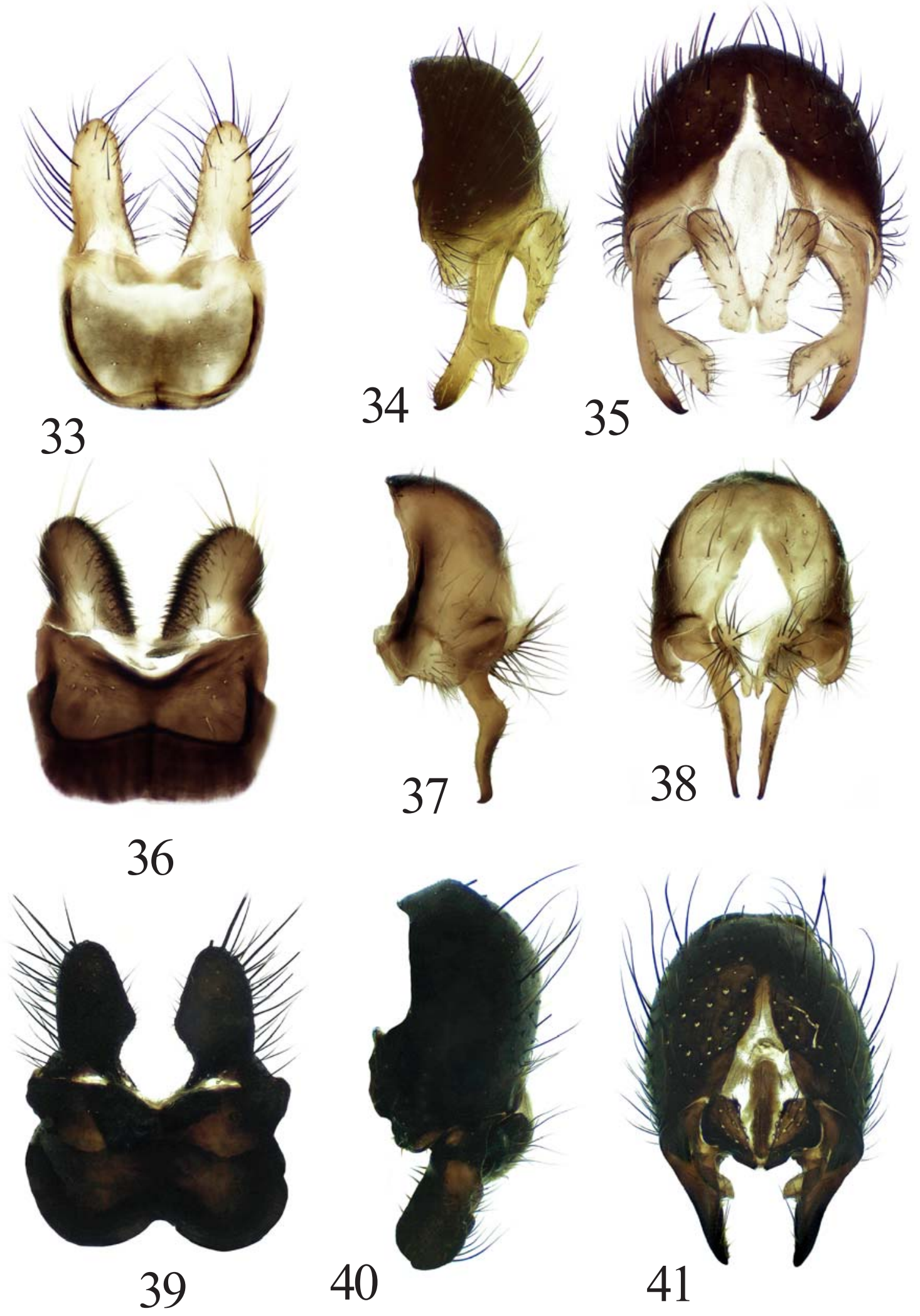

Figs 33-41. Cordilura albipes (Fallén) (33-35), C. amurensis Ozerov (36-38) and C. atrata (Zetterstedt) (39-41): 33, 36, 39 - male sternite 5; 34, 37, 40 - epandrium and surstyli, lateral view; 35, 38, 41 - same, dorsal view.

Рис. 33-41. Cordilura albipes (Fallén) (33-35), C. amurensis Ozerov (36-38) и C. atrata (Zetterstedt) (39-41): 33, 36, 39 стернит 5 самца; $34,37,40$ - эпандрий и сурстили, сбоку; $35,38,41$ - то же, сверху. 
flection. Postcranium yellow completely or yellow in lower part and black in upper half or third. 2 orbital and 2-4 frontal setae present. Antenna pale yellow. Postpedicel approximately 2 times as long as wide. Arista plumose, longest hairs approximately as long as width of postpedicel. Palpus yellow. Thorax yellow in ground colour; scutum varies in colour from shiny black completely to almost yellow completely, usually yellow with two blackish stripes along dorsocentral lines. Acrostichals setulose in two rows, dorsocentrals $3+3$ (including scapular seta), intra-alars $(0-1)+(0-2)$, supra-alars $1+2,2$ postpronotals. Scutellum with a pair of strong discal setae only; apical scutellar setae as setulae or absent (Fig. 12). Legs shining, usually yellow completely, sometimes mid and hind femora slightly darkened. Wing tinged with brownish; veins blackish. Vein $\mathrm{R}_{1}$ setulose on apical third of dorsal surface. Abdomen from yellow to black, shining or subshining. Male sternite 4 rectangular, longer than wide. Male sternite 5, epandrium and surstyli as in Figs 33-35. Female ovipositor as in Figs 21-23.

DISTRIBUTION. Russia: Adygea, Altai, Arkhangelsk Oblast, Buryatia, Chelyabinsk Oblast, Crimea, Dagestan, Irkutsk Oblast, Karelia, Kemerovo Oblast, Krasnodar Krai, Krasnoyarsk Krai, Leningrad Oblast, Mordovia, Moscow Oblast, Murmansk Oblast, Nizhegorod Oblast, North Ossetia - Alania, Novgorod Oblast, Novosibirsk Oblast, Primorsky Krai, Rostov Oblast, Ryazan' Oblast, Sakhalin Oblast, Tatarstan, Tomsk Oblast, Tyumen' Oblast, Tuva, Yakutia, Zabaykalsky Krai. - Europe, including Belarus (first record), Armenia (first record), Georgia (first record), Iran [Khaghaninia, Gharajedaghi, 2014: 391], Mongolia, Japan [Iwasa, 2020: 472].

\section{Cordilura amurensis Ozerov, 2007 Figs 36-38.}

amurensis Ozerov, 2007b: 123 (Cordilura). Type-locality: Zeya Town (Russia, Amur Oblast).

REMARK. This species was described and recorded in Russia from Amur Oblast and Khabarovsk Krai [Ozerov, 2007b: 123; Ozerov, Krivosheina, 2014: 207].

MATERIAL EXAMINED. Amur Oblast: Zeya Town

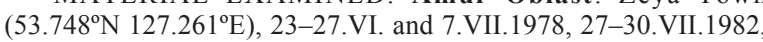
A. Shatalkin (2 ○ $^{7}, 7$ 70, ZMUM); Zeysky Nature Reserve, cordon "34 km" (53.989 $\left.\mathrm{N} 127.073^{\circ} \mathrm{E}\right), 27$ and 28.VII.1981, A.L. Ozerov, A. Shatalkin (1 $\sigma^{7}, 2$ 9 , ZMUM); Zeysky Nature Reserve, cordon "52 km" $\left(54.087^{\circ} \mathrm{N} 126.871^{\circ} \mathrm{E}\right), 2-9$. VII.1981, A. Shatalkin (3 $\sigma^{\top} \sigma^{7}, 1$ ㅇ, ZMUM)

DESCRIPTION. Male. Female. Body-length 3.6$5.1 \mathrm{~mm}$. Head. Frontal vitta yellow, matt; fronto-orbital plate yellow, whitish dusted. Face, parafacial and gena whitish. Postcranium greyish dusted, blackish or yellow in upper half and whitish in lower half. 2 orbital and 2-3 frontal setae present. Scapus and pedicel yellow, postpedicel blackish in male and yellow in female. Postpedicel approximately 2 times as long as wide. Arista nearly bare. Palpus pale yellow. Thorax reddish yellow completely or brownish in upper part, delicately greyish dusted. Acrostichals absent, dorso- centrals $3+3$ (including scapular seta), intra-alars $1+0$, supra-alars $1+2,1-2$ postpronotals. Scutellum with a pair of strong discal setae and a pair of apical setulae. Legs yellow, but usually tarsomere 5 dark brownish. Wing tinged with light brownish; veins brownish. Vein $\mathrm{R}_{1}$ setulose on apical third of dorsal surface. Abdomen blackish greyish dusted; female tergite 6 yellow. Male sternite 4 rectangular, longer than wide. Male sternite 5, epandrium and surstyli as in Figs 36-38.

DISTRIBUTION. Russia: Amur Oblast, Khabarovsk Krai.

\section{Cordilura atrata (Zetterstedt, 1846)}

Figs 39-41.

atrata Zetterstedt, 1846: 2002 (Cordylura). Type-locality: "Lapponia Lulensi ad Qvickjock ... ad Råbäcken ... in Jemtlandia ad Skalstugan \& in ipso jugo alpino Norwegiae inter Kongsstuen \& Suulstuen" (Sweden, Norway).

REMARK. This species was noted for European part of Russia (Kola Peninsula, Komi, Polar Ural) [Gorodkov, 1970: 446] and Siberia without indicating specific locality [Gorodkov, 1986: 14], also from Far East, including Khabarovsk Krai and Magadan Oblast [Ozerov, Krivosheina, 2014: 207], and Yakutia [Bagachanova et al., 2016: 779].

MATERIAL EXAMINED. Arkhangelsk Oblast: Nenetsky Nature Reserve, cordon "Bol'shoy Gusinets" $\left(68.175^{\circ} \mathrm{N}, 53.645^{\circ} \mathrm{E}\right)$, 10.VII.2008, N.E. Vikhrev (3 $\sigma^{\top} \sigma^{\top}, 1$ ㅇ, ZMUM); the lower reaches of the River Pechora, Kashin I. $\left(68.242^{\circ} \mathrm{N}, 53.856^{\circ} \mathrm{E}\right)$, 10.VII.2008, A.L. Ozerov (2 $0^{7} \sigma^{7}$, ZMUM); Nar'yan-Mar $\left(67.636^{\circ} \mathrm{N}\right.$ $53.031^{\circ} \mathrm{E}$ ), 2, 4 and 20.VIII.1978, Gorodkov ( $8 \sigma^{\top} \sigma^{\top}, 5$ 우, ZISP); $73 \mathrm{~km} \mathrm{NW}$ of Nar'yan-Mar (ca. $67.981^{\circ} \mathrm{N} 51.608^{\circ} \mathrm{E}$ ), 3.VIII.1978, Gorodkov (7 $0^{7} \sigma^{7}, 6$ 우, ZISP); $70 \mathrm{~km} \mathrm{~N}$ of Nar'yan-Mar (ca. $\left.68.204^{\circ} \mathrm{N} 53.627^{\circ} \mathrm{E}\right), 18$.VIII.1978, Gorodkov (2 $\bigcirc^{7} \sigma^{7}$, ZISP); Chukotka: Meynypil'gyno env. (62.538 $\left.\mathrm{N}, 177.051^{\circ} \mathrm{E}\right)$, 9.VII.2013, P.C. Tomkovich (1 $\sigma^{7}, 1$ \% , ZMUM); Meynypil'gyno env. $\left(62.553^{\circ} \mathrm{N}\right.$ $\left.177.038^{\circ} \mathrm{E}\right)$, 13.VII.2015, P.S. Tomkovich (3 $\sigma^{7} \sigma^{7}$, ZMUM); bank of the River Anadyr $\left(64.72^{\circ} \mathrm{N} 175.21^{\circ} \mathrm{E}\right), 25$. VI.-19.VII.2014, A. Barkalov (1 $\sigma^{7}$, ISEA); $20 \mathrm{~km} \mathrm{SSW}$ of Iul'tin $\left(67.724^{\circ} \mathrm{N} 178.515^{\circ} \mathrm{W}\right)$, 22.VII.1963, Gorodkov (1 \&, ZISP); Kamchatka Krai: Uzon $\left(54.503^{\circ} \mathrm{N} 159.990^{\circ} \mathrm{E}\right)$, 9.VII.1987, L. Lobkova (1 フ', ZMUM); Komi: Vorkuta $\left(67.5^{\circ} \mathrm{N} 64.0^{\circ} \mathrm{E}\right), 19-25 . \mathrm{VII} .2010, \mathrm{~N}$. Vikhrev (2 오, ZMUM); Shchel'yabozh $\left(66.29^{\circ} \mathrm{N} 56.45^{\circ} \mathrm{E}\right), 14$ and 15.VIII.1978, Gorodkov (1 $\sigma^{7}, 1$ क, ZISP); Sivaya Maska station $\left(66.675^{\circ} \mathrm{N} 62.569^{\circ} \mathrm{E}\right), 5 \mathrm{~km} \mathrm{NW}, 14 . \mathrm{VII} .1961$, Gorodkov (1 $\bigcirc^{7}$, ZISP); Krasnoyarsk Krai: Medvezhiy $\left(69.284^{\circ} \mathrm{N} 88.148^{\circ} \mathrm{E}\right)$, Noril'sk env., 6.VII.1967, Gorodkov ( $3 \mathrm{\sigma}^{\top} \mathrm{O}^{\top}, 3$ 우, ZISP); Noril'sk env. (69.339 $\left.{ }^{\circ} \mathrm{N} 88.214^{\circ} \mathrm{E}\right), 26$. VIII.1975, Gorodkov (1 $\sigma^{7}$, ZISP); $104 \mathrm{~km}$ NNW of Noril'sk, River Nizhnyaya Agapa (ca. $70.097^{\circ} \mathrm{N}$ $\left.86.688^{\circ} \mathrm{E}\right), 26$.VII.1973, Sukacheva, Zherikhin (1 $\sigma^{7}$, ZISP); Agapa $\left(71.421^{\circ} \mathrm{N} 89.2512^{\circ} \mathrm{E}\right)$, River Pyasina, 15.VII.1967, Gorodkov (3 ○ $^{\top}$, ZISP); Dudinka $\left(69.407^{\circ} \mathrm{N} 86.175^{\circ} \mathrm{E}\right), 3$. VII.1967, Gorodkov (1 $\sigma^{7}$, ZISP); Igarka $\left(67.468^{\circ} \mathrm{N} 86.581^{\circ} \mathrm{E}\right), 30 . \mathrm{VI}$. and 1.VII.1967, Gorodkov (4 $\sigma^{7} \sigma^{7}, 2$ 우, ZISP); Mordovia: Mordovsky Nature Reserve, Cordon Steklyanny (54.894 $\left.{ }^{\circ} \mathrm{N}, 43.601^{\circ} \mathrm{E}\right), 12-15$. VII.2020, K. Tomkovich (1 $\sigma^{7}$, ZMUM); Moscow Oblast: Andreevskoe $\left(55.967^{\circ} \mathrm{N} 35.609^{\circ} \mathrm{E}\right)$, 6.V.2009, A.L. Ozerov (2 $\sigma^{7} \sigma^{7}$, ZMUM); Dmitrov env. $\left(56.316^{\circ} \mathrm{N} 37.725^{\circ} \mathrm{E}\right), 27 . V .2009$, N. Vikhrev $\left(1 \sigma^{7}, 1\right.$ +, ZMUM); $35 \mathrm{~km} \mathrm{NNE}$ of Moscow $\left(56.200^{\circ} \mathrm{N} 37.833^{\circ} \mathrm{E}\right)$, 26.VI.1993, A.L. Ozerov (1 $\sigma^{\top}$, ZMUM); Murmansk Oblast: Lovozero ( $\left.68.005^{\circ} \mathrm{N} 35.016^{\circ} \mathrm{E}\right), 14$.VIII.1981, Gorodkov (1 9 , ZISP); Leningrad Oblast: Rozhdestveno $\left(59.322^{\circ} \mathrm{N} 29.946^{\circ} \mathrm{E}\right)$, 4.VI.1956, A. Stackelberg $\left(1 \mathrm{O}^{\top}, 1\right.$ O, $\left.\mathrm{ZISP}\right)$; Yashchera $\left(58.894^{\circ} \mathrm{N} 29.820^{\circ} \mathrm{E}\right)$, 23.VII.1957, A. Stackelberg (1 ऽ, ZISP); Lakhta $\left(59.991^{\circ} \mathrm{N}\right.$ $30.159^{\circ}$ E), 15.VI.1919, A. Stackelberg (1 9 , ZISP); Novosibirsk Oblast: Zherebtsovo env. $\left(55.125^{\circ} \mathrm{N} 83.256^{\circ} \mathrm{E}\right)$, 31.V.2008, O. Kosterin ( $\Im^{\top}$, ZMUM); Sakhalin Oblast: Iturup I., Kurilsk 


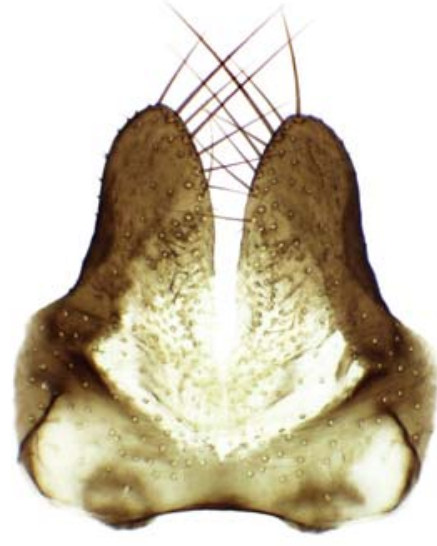

42
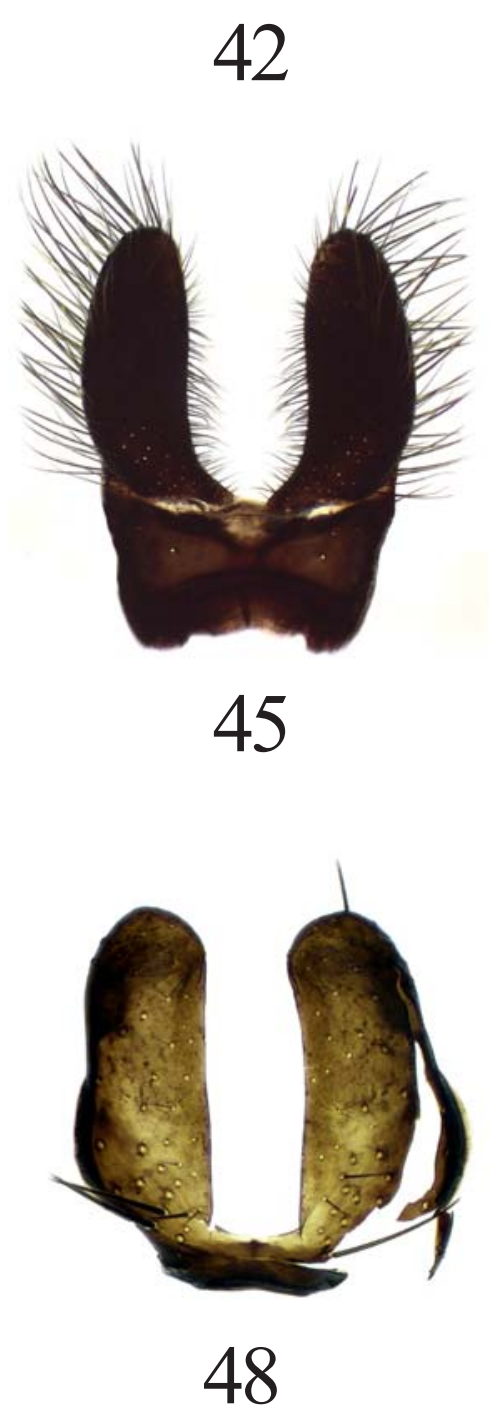
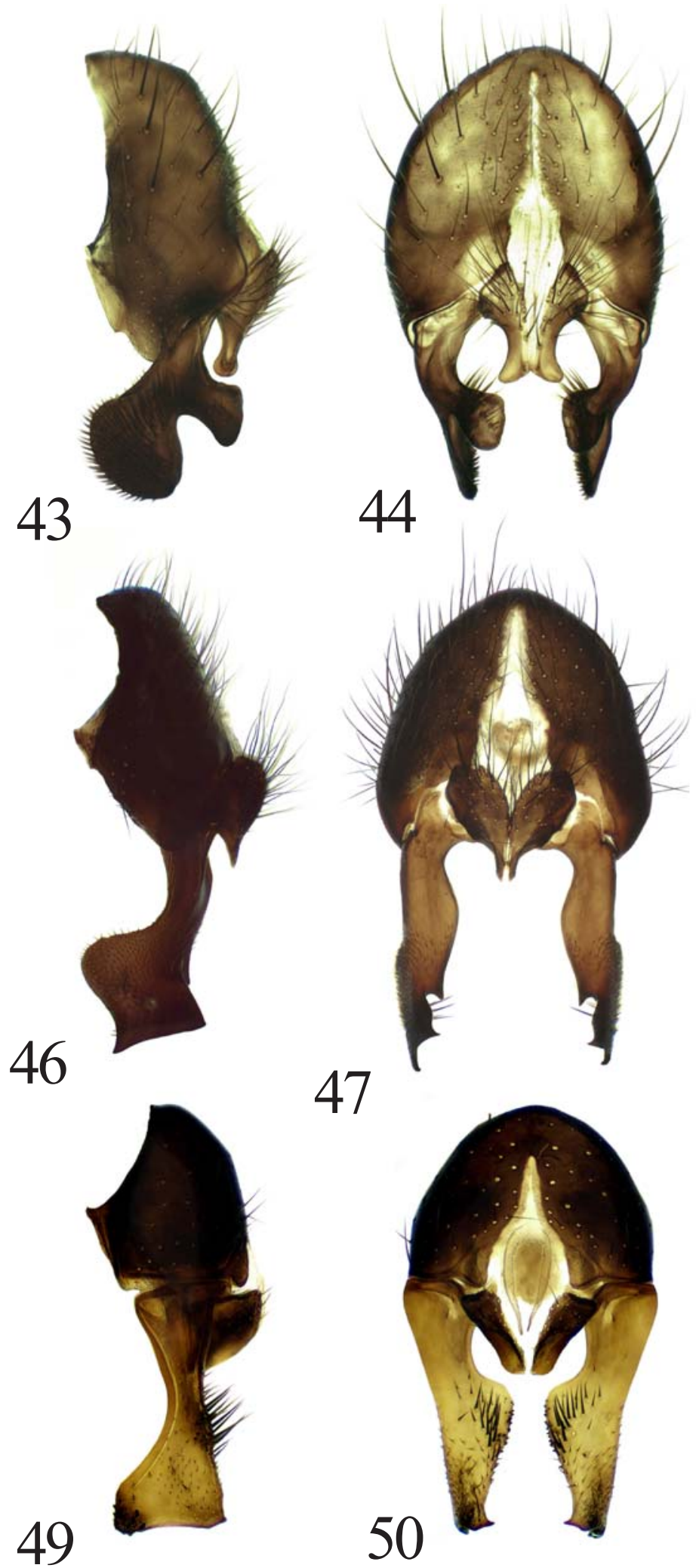

Figs 42-50. Cordilura bicoloripes Ozerov (42-44), C. ciliata (Meigen) (45-47) and C. fulvifrons Ozerov (48-50): 42, 45, 48 - male sternite $5 ; 43,46,49$ - epandrium and surstyli, lateral view; 44, 47, 50 - same, dorsal view. 48-50 - after Ozerov \& Krivosheina, 2017, figs 4-6.

Puc. 42-50. Cordilura bicoloripes Ozerov (42-44), C. ciliata (Meigen) (45-47) и C. fulvifrons Ozerov (48-50): 42, 45, 48 стернит 5 самца; 43, 46, 49 - эпандрий и сурстили, сбоку; 44, 47, 50 - то же, сверху. 48-50 - по Ozerov \& Krivosheina, 2017, figs $4-6$. 
$\left(45.226^{\circ} \mathrm{N} 147.881^{\circ} \mathrm{E}\right), 25 . \mathrm{VI} .1968, \mathrm{~V}$. Richter $\left(2 \mathrm{O}^{7} 0^{7}, 1\right.$,, ZISP); Tyumen' Oblast: Saranpaul' $\left(64.257^{\circ} \mathrm{N} 60.917^{\circ} \mathrm{E}\right), 23 . V I .1989$, Malozemov $\left(1 \bigcirc^{7}\right.$, ZISP); Nyda $\left(66.628^{\circ} \mathrm{N} 72.922^{\circ} \mathrm{E}\right)$, 3.VIII. 1977 , Gorodkov (2 우, ZISP); $75 \mathrm{~km}$ WSW of Samburg (ca. $67.035^{\circ} \mathrm{N}$ $\left.76.541^{\circ} \mathrm{E}\right)$, 7.VIII.1976, Gorodkov (1 9 , ZISP); Yakutia: airport Saskylach $\left(71.934^{\circ} \mathrm{N} 114.083^{\circ} \mathrm{E}\right), 24$. VII.1988, Gorodkov $\left(2 \mathrm{O}^{7} \mathrm{O}^{7}\right.$, ZISP); Saskylakh env. (71.966 $\left.\mathrm{N} 114.094^{\circ} \mathrm{E}\right), 24$ and 27.VIII.1988, Gorodkov (2 우, ZISP); Ust'-Kuyga $\left(70.000^{\circ} \mathrm{N} 135.562^{\circ} \mathrm{E}\right), 26$.VII. 1974, Gorodkov (3 $0^{7} \sigma^{7}, 1$ ㅇ, ZISP).

DIAGNOSTIC DESCRIPTION. Male. Female. Body-length 4.6-5.8 mm. Head. Frontal vitta black or dark brown, frequently reddish yellow apically, with whitish reflection; fronto-orbital plate black, greyish dusted. Face and parafacial yellowish with whitish reflection. Gena brownish. Postcranium black, grey dusted. 3 orbital and 2-3 frontal setae present. Antenna black. Postpedicel approximately 2 times as long as wide. Arista pubescent, the longest hairs approximately equal to $1 / 3-1 / 2$ width of postpedicel. Palpus black. Thorax black, grey dusted. Acrostichals setulose in two rows, dorsocentrals $3+3$ (including small scapular seta), intra-alars $1+2$, supra-alars $1+2,2$ postpronotals. Scutellum with a pair of strong discal and a pair of strong apical setae. Legs greyish dusted. Fore coxa black; femora, tibiae and tarsi of all legs black. Wing tinged with brownish; veins blackish. Vein $\mathrm{R}_{1}$ setulose on apical third of dorsal surface. Abdomen black, grey dusted. Male sternite 4 rectangular, wider than long. Male sternite 5, epandrium and surstyli as in Figs 39-41.

DISTRIBUTION. Russia: Arkhangelsk Oblast, Chukotka, Kamchatka Krai, Komi, Krasnoyarsk Krai, Mordovia, Moscow Oblast, Murmansk Oblast, Leningrad Oblast, Novosibirsk Oblast, Sakhalin Oblast, Tyumen' Oblast, Yakutia. - Europe, North America.

\section{Cordilura bicoloripes Ozerov, 1997} Figs 42-44.

bicoloripes Ozerov, 1997: 1424 (Cordilura). Type-locality: 40 km SE of Ussuriysk (Russia, Primorsky Krai).

REMARK. This species was described and recorded in Russia from Primorsky Krai only [Ozerov, 1997: 1424; Ozerov, Krivosheina, 2014: 207].

MATERIAL EXAMINED. Primorsky Krai: Kamenushka (43.634 $\left.{ }^{\circ} \mathrm{N} 132.222^{\circ} \mathrm{E}\right), 6$.VIII.1984, 6-25.VIII., 3-4.IX.1987, A Shatalkin (4 ○ๆ $\sigma^{7}, 4$ 오, ZMUM); same place, 4.IX.1983, A.L. Ozerov (1 $\sigma^{7}$, ZMUM); Kedrovaya Pad' Nature Reserve $\left(43.104^{\circ} \mathrm{N}\right.$ $\left.131.512^{\circ} \mathrm{E}\right), 17$ and 19.VIII.1962, Nartshuk ( $\sigma^{\top}, 1$ ․ ZISP); Kievka $\left(42.904^{\circ} \mathrm{N} 133.701^{\circ} \mathrm{E}\right), 6-7 . I X .1980$, A. Shatalkin $\left(1 \sigma^{7}, 2\right.$ + , ZMUM); Sudzukhinskiy [=Lazovsky] Nature Reserve, Tachingou $\left(43.023^{\circ} \mathrm{N} 134.136^{\circ} \mathrm{E}\right)$, 30.IX., 2-9.X.1948, Gussakovskiy $\left(5\right.$ +o, ZMUM); Tigrovoy [=Tigrovoe] $\left(43.189^{\circ} \mathrm{N} 132.894^{\circ} \mathrm{E}\right)$, 26.VIII.1962, Nartshuk ( $\sigma^{\top}, 1$ \%, ZISP); riverhead of the River Chapingou $[=$ Krounovka], tributary of the River Shufan $[=$ Borisovka] (ca. $\left.43.615^{\circ} \mathrm{N} 131.541^{\circ} \mathrm{E}\right), 1 . V I I .1962$, Nartshuk (1 $\sigma^{\top}, 1$ 웅, ZISP); Vladivostok env., Okeanskaya $\left(43.234^{\circ} \mathrm{N} 132.014^{\circ} \mathrm{E}\right)$, 3.IX.1978, A. Zinoviev (1 ơ', ZISP).

DIAGNOSTIC DESCRIPTION. Male. Female. Body-length 6.0-9.2 mm. Head. Frontal vitta and fronto-orbital plate black, with whitish reflection. Face and parafacial yellow with whitish reflection in male and black with whitish reflection in female. Gena black in both sexes. Postcranium black, grey dusted. 2 orbital and 3-4 frontal setae present. Antenna brownish or blackish. Postpedicel approximately 2.5 times as long as wide. Arista pubescent, the longest hairs approximately equal to $1 / 3-1 / 2$ width of postpedicel. Palpus yellow. Thorax black, delicately grey dusted, but scutum shining. Acrostichals absent, dorsocentrals $2+3$ (including small scapular seta), intra-alars absent, supra-alars $0+2$, postpronotals absent. Scutellum with a pair of strong discal setae only; apical scutellar setae absent. Legs shining. Femora of all legs bicolor: black in apical part and yellow in basal half or third; tibiae and tarsomeres 1-3 of all legs yellow, tarsomeres 4 and 5 of all legs brown or black. Wing tinged with brownish, darkened in apical third; veins yellowish. Vein $\mathrm{R}_{1}$ bare on apical third of dorsal surface. Abdomen black, shining. Male sternite 4 rectangular, slightly longer than wide. Male sternite 5, epandrium and surstyli as in Figs 42-44.

DISTRIBUTION. Russia: Primorsky Krai.

\section{Cordilura ciliata (Meigen, 1826)} Figs 6, 45-47.

ciliata Meigen, 1826: 231 (Cordylura). Type-locality: not given.

REMARK. Recorded for European part of Russia without indicating specific locality [Gorodkov, 1970: 446, 1986: 14] and from Karelia [Humala, Polevoi, 2009: 71].

MATERIAL EXAMINED. Altai: Kosh-Agach, plato Ukok, $2400 \mathrm{~m}$, Lake Muzdy-Bulak env. (49.26 $\left.{ }^{\circ} \mathrm{N}, 87.65^{\circ} \mathrm{E}\right), 10-$ 12.VII.2008, A. Barkalov, ( $\sigma^{\top} \sigma^{\top}, 2$ 우, ISEA); Verkh-Kukuya env., floodplain of the River Sema, $980 \mathrm{~m},\left(51.516^{\circ} \mathrm{N}, 85.566^{\circ} \mathrm{E}\right)$, 17.VII.2011, V. Sorokina ( $\sigma^{7}$, ISEA); Cherga env., floodplain of the River Sema, $516 \mathrm{~m},\left(51.516^{\circ} \mathrm{N} 85.566^{\circ} \mathrm{E}\right), 16 . \mathrm{VII} .2011, \mathrm{~V}$. Sorokina (2 $\sigma^{\top} \sigma^{7}, 1$ \% ISEA); Ulus-Cherga $\left(51.519^{\circ} \mathrm{N} 85.459^{\circ} \mathrm{E}\right)$, 27.VII.2008, O. Kosterin (1 O, ZMUM); Lake Teletskoe, 25 km S of Yogach, Obogo Pass $\left(51.55^{\circ} \mathrm{N} 87.26^{\circ} \mathrm{E}\right), 1077 \mathrm{~m}, 11 . \mathrm{VIII} .2017$, V.S. Sorokina ( $1 \sigma^{7}$, ISEA); Arkhangelsk Oblast: Solvychegodsk $\left(61.342^{\circ} \mathrm{N} 46.913^{\circ} \mathrm{E}\right)$, 13.VIII.2010, D. Gavryushin (3 $\sigma^{7} \sigma^{\top}, 7$, 7 , ZMUM); Arkhangelsk $\left(64.546^{\circ} \mathrm{N} 40.567^{\circ} \mathrm{E}\right), 4$.VIII.2010, D. Gavryushin $\left(1 \sigma^{7}, 2\right.$ 우, ZMUM); Talagi $\left(64.620^{\circ} \mathrm{N} 40.648^{\circ} \mathrm{E}\right)$, 13.VIII.1970, Gorodkov (1 $\sigma^{7}$, ZISP); Bashkiria: Beloretsk $\left(53.963^{\circ} \mathrm{N} 58.765^{\circ} \mathrm{E}\right), 12$. VIII.2012, D. Gavryushin $\left(1 \bigcirc^{\top}, 2\right.$ 우, ZMUM); Beloretsk distr., River Nura $\left(54.051^{\circ} \mathrm{N} 58.268^{\circ} \mathrm{E}\right), 10,13$ and 16.VII.2015, D. Gavryushin (5 $\sigma^{7} \sigma^{7}$, ZMUM); Chelyabinsk Oblast: Taganay $\left(55.277^{\circ} \mathrm{N} 59.795^{\circ} \mathrm{E}\right), 18-24$.VII.2008, K. Tomkovich (1 9 , ZMUM); Karelia: Primorskiy $\left(66.546^{\circ} \mathrm{N} 33.103^{\circ} \mathrm{E}\right)$, 3-5.VII.2010, A.L. Ozerov (3 OO ZMUM); Kaskesnavolok $\left(61.594^{\circ} \mathrm{N} 33.323^{\circ} \mathrm{E}\right), 20 . \mathrm{VI} .1979$, Gorodkov (1 $\sigma^{\top}$, ZISP); Kemerov Oblast: Novokuznetsk env. $\left(55.757^{\circ} \mathrm{N} 87.148^{\circ} \mathrm{E}\right)$, 29.VI.2011, O. Kosterin (1 $\sigma^{7}$, ZMUM); Khakassia: Kubayka $\left(52.33^{\circ} \mathrm{N} 89.82^{\circ} \mathrm{E}\right), 620 \mathrm{~m}, 10-13 . V I I .2017, \mathrm{~N}$. Vikhrev $\left(1 \sigma^{7}\right.$, ZMUM); Komi: Ust-Tsilma $\left(65.440^{\circ} \mathrm{N} 52.153^{\circ} \mathrm{E}\right), 9$ and 11.VIII.1978, Gorodkov (10 $\sigma^{7} \sigma^{7}, 10$ 우, ZISP); Blagoevo $\left(63.424^{\circ} \mathrm{N}, 47.952^{\circ} \mathrm{E}\right), 13$.VII.1996, Gorodkov (1 $\sigma^{7}$, ZISP); Kursk Oblast: Oboyan' $\left(51.191^{\circ} \mathrm{N} 36.312^{\circ} \mathrm{E}\right), 20-21$.VII.2007, A.L. Ozerov (1 $\sigma^{7}$, ZMUM); Leningrad Oblast: Tolmachevo $\left(58.856^{\circ} \mathrm{N}\right.$ $\left.29.895^{\circ} \mathrm{E}\right), 24$.VIII.1961, Rudenko (1 $\sigma^{7}$, ZISP); same place, 9.VII.1935, Rohdendorf (4 $\sigma^{7} \sigma^{7}$, ZISP); Gor'kovskoe, Lake Lebyazh'e $\left(60.312^{\circ} \mathrm{N} 29.532^{\circ} \mathrm{E}\right), 24$.VIII.2003, A. Ovchinnikov (3 $0^{7} \sigma^{7}, 2$ OO. ZISP); Luga (ca. $58.73^{\circ} \mathrm{N} 29.84^{\circ} \mathrm{E}$ ), 7, 24.VII. and 19.VIII.1952, 15, 17.VI.1954, A. Stackelberg (4 Oフ $\sigma^{7}, 4$ 오, ZISP); Preobrazhenskay [=Tolmachovo] $\left(58.871^{\circ} \mathrm{N} 29.899^{\circ} \mathrm{E}\right), 13 . \mathrm{VI} .1898$, Pleske (1 9 , ZISP); Rozhdestveno $\left(59.322^{\circ} \mathrm{N} 29.946^{\circ} \mathrm{E}\right), 15,23$, 28.VI. and 1.VII.1956, A. Stackelberg (7 $\sigma^{\top} \sigma^{\top}, 3$ 우, ZISP); same

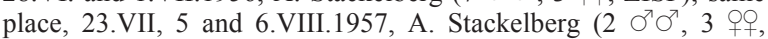
ZISP); Shuvalovo $\left(60.047^{\circ} \mathrm{N} 30.283^{\circ} \mathrm{E}\right), 1,21$ and 27.VI.1897, 16 and 21.VI.1915, Yakobson (1 $\sigma^{\top}, 8$ 우, ZISP); Yashchera $\left(58.894^{\circ} \mathrm{N}\right.$ $\left.29.820^{\circ} \mathrm{E}\right), 27 . \mathrm{VII} ., 1,2,15$ and $22 . \mathrm{VIII} .1959,22 . \mathrm{VII}$. and 


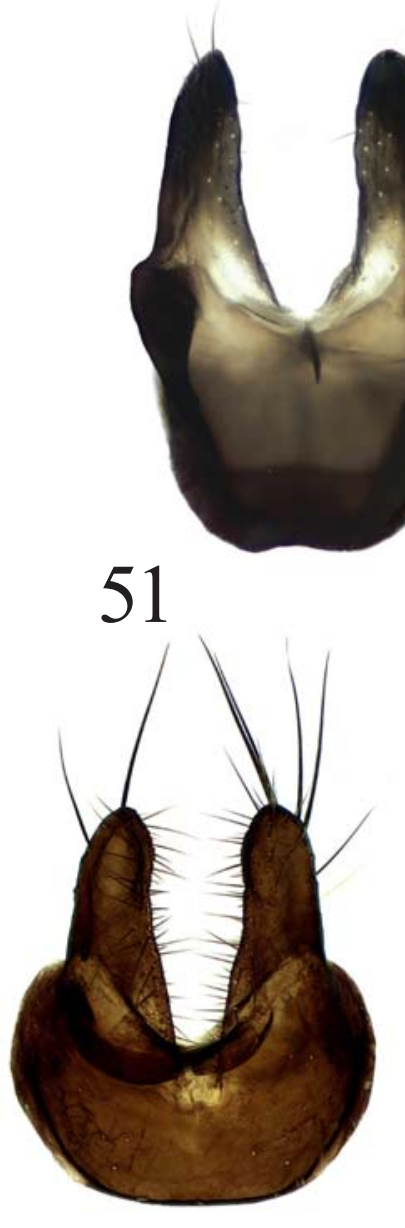

\section{4}

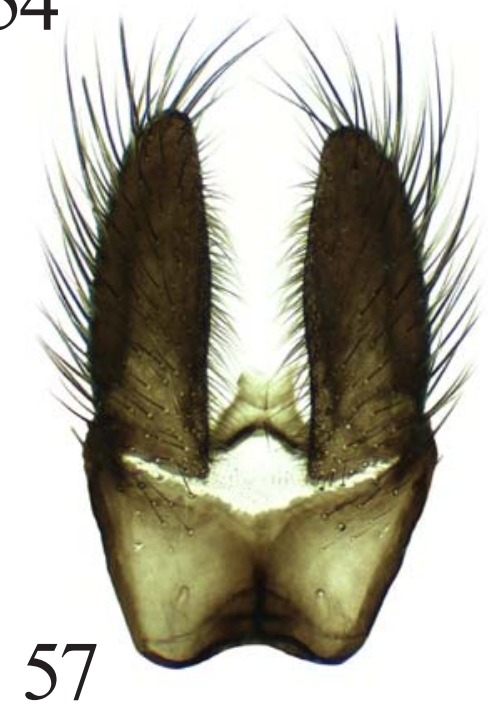

52
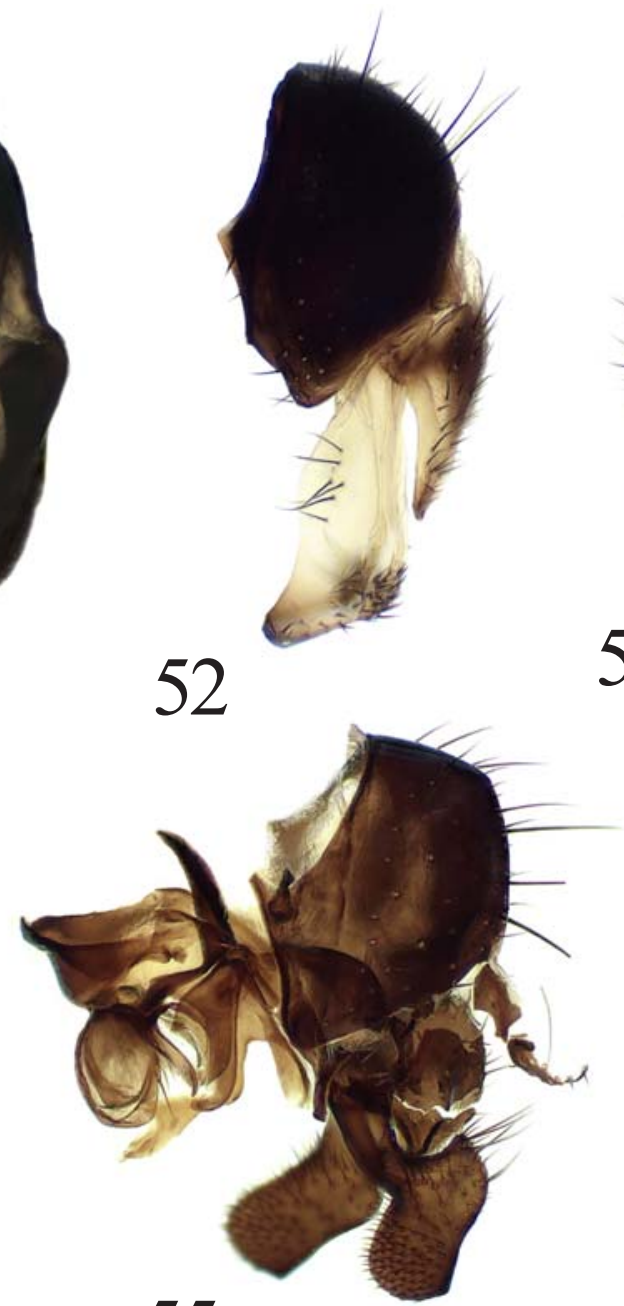

55

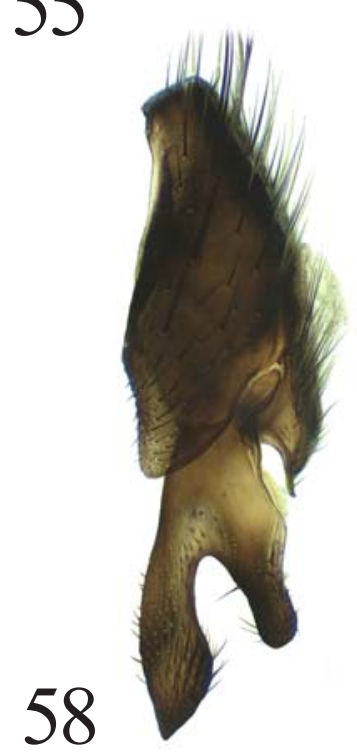

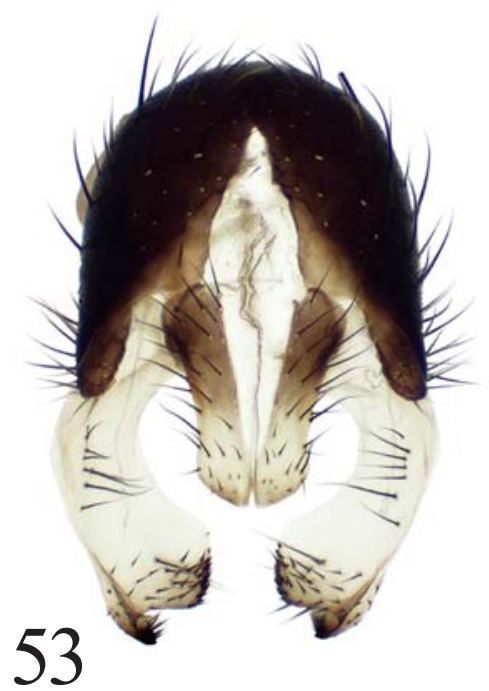
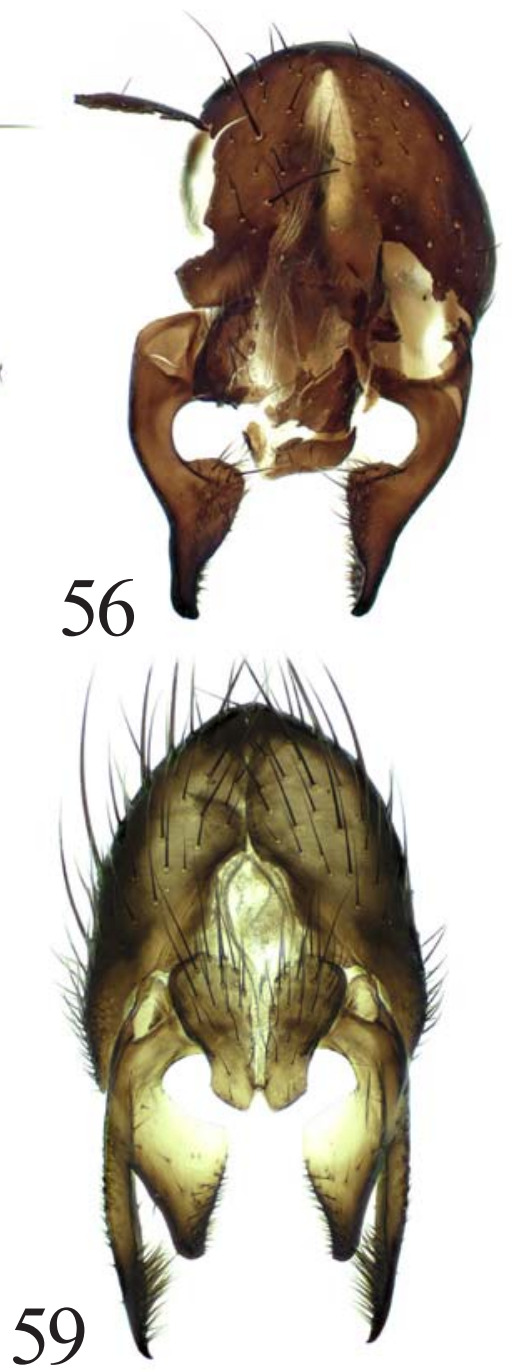

Figs 51-59. Cordilura fuscipes (Zetterstedt) (51-53), C. grunini Ozerov et Krivosheina (54-56) and C. impudica Rondani (57-59): $51,54,57$ - male sternite 5; 52, 55, 58 - epandrium and surstyli, lateral view; 53, 56, 59 — same, dorsal view. 54-56 - after Ozerov \& Krivosheina, 2017, figs 1-3; 58 - after Ozerov \& Krivosheina, 2014, fig. 13.

Pис. 51-59. Cordilura fuscipes (Zetterstedt) (51-53), C. grunini Ozerov et Krivosheina (54-56) и C. impudica Rondani (57-59): 51, 54, 57 - стернит 5 самца; 52, 55, 58 - эпандрий и сурстили, сбоку; 53, 56, 59 - то же, сверху. 54-56 - по Ozerov \& Krivosheina, 2017, figs 1-3; 58 - по Ozerov \& Krivosheina, 2014, fig. 13. 


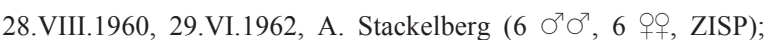
Moscow and Moscow Oblast: Izmaylovo $\left(55.786^{\circ} \mathrm{N} 37.835^{\circ} \mathrm{E}\right)$

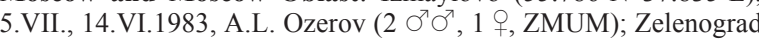
$\left(55.986^{\circ} \mathrm{N} 37.202^{\circ} \mathrm{E}\right), 3$. VIII.1999, A. Gusakov (1 $\sigma^{7}$, ZMUM); 20 km WSW of Volokolamsk $\left(55.983^{\circ} \mathrm{N} 35.616^{\circ} \mathrm{E}\right)$, 13.VII.1995, A.L. Ozerov (1 $\sigma^{7}$, ZMUM); $35 \mathrm{~km}$ NNE of Moscow $\left(56.200^{\circ} \mathrm{N}\right.$

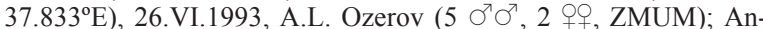
dreevskoe $\left(55.985^{\circ} \mathrm{N} 35.620^{\circ} \mathrm{E}\right), 21$. VII.2006, A.L. Ozerov (1 $\sigma^{7}$, ZMUM); Dmitrov env. $\left(56.316^{\circ} \mathrm{N} 37.725^{\circ} \mathrm{E}\right), 19 . \mathrm{VI} ., 10 . \mathrm{VII}$, 19.VIII.2007, N. Vikhrev (3 $\sigma^{\top} \sigma^{\top}, 1$. ZMUM); Mamontovka $\left(55.999^{\circ} \mathrm{N} 37.816^{\circ} \mathrm{E}\right)$, 5.VI.1956, Yu. Chernov (1 ऽ $\left.{ }^{\top}, \mathrm{ZMUM}\right)$ Naro-Fominsk $\left(55.39^{\circ} \mathrm{N} 36.735^{\circ} \mathrm{E}\right)$, 22.VI.2011, D. Gavryushin (1 $\left.\sigma^{7}, \mathrm{ZMUM}\right)$; Podrezkovo $\left(55.941^{\circ} \mathrm{N} 37.332^{\circ} \mathrm{E}\right), 24 . \mathrm{VII} .1956, \mathrm{Yu}$ Chernov (1 $\sigma^{\top}$, ZMUM); Stepan'kovo $\left(55.004^{\circ} \mathrm{N} 35.624^{\circ} \mathrm{E}\right)$, 9.VII.2006, 3.VI.2007, A.L. Ozerov (3 $\sigma^{7} \sigma^{\top}, 2$ 우, ZMUM); Murmansk Oblast: Vud'yavr Lake Basin (ca. $67.646^{\circ} \mathrm{N} 33.644^{\circ} \mathrm{E}$ ), 14.VII.1931 and 8.VII.1936, Fridolin (2 90 , ZISP); Novosibirsk: Academgorodok $\left(54.87^{\circ} \mathrm{N} 83.05^{\circ} \mathrm{E}\right), 18-19 . \mathrm{VI} .2016$, N. Vikhrev (2 $\sigma^{\top} \sigma^{7}$, ZMUM); Omsk Oblast: Syropyatskoe $\left(55.047^{\circ} \mathrm{N} 73.874^{\circ} \mathrm{E}\right)$, $32 \mathrm{~km}$ E of Omsk, 27.VIII.1985, Gorodkov (1 9 , ZISP); Pskov Oblast: Aparino $\left(56.958^{\circ} \mathrm{N} 29.966^{\circ} \mathrm{E}\right), 28 . \mathrm{VI} .1915, \mathrm{~N}$. Kuznetsov (1 $\sigma^{7}$, ZISP); Lake Dubets $\left(56.966^{\circ} \mathrm{N} 30.006^{\circ} \mathrm{E}\right), 19$.VI.1915, N. Kuznetsov (1 ${ }^{\prime}$, ZISP); Pokrovskoe $\left(58.311^{\circ} \mathrm{N} 28.960^{\circ} \mathrm{E}\right), 11 . \mathrm{VI} .1915, \mathrm{~N}$ Kuznetsov (1 $\sigma^{7}$, ZISP); same place, 13.VI.1960, Nartshuk (2 $\sigma^{7} \sigma^{7}$ ZISP); Ryazan' Oblast: Kasimov $\left(54.94^{\circ} \mathrm{N} 41.34^{\circ} \mathrm{E}\right)$, 25.VII.2013, N. Vikhrev (1 q, ZMUM); Smolensk Oblast: Smolenskoe poozer'e National Park $\left(55.511^{\circ} \mathrm{N} 31.839^{\circ} \mathrm{E}\right), 30 . \mathrm{VI} .1992$, Zlobin (1 $\left.\sigma^{7}, \mathrm{ZISP}\right)$ Sverdlovsk Oblast: Polevskoy env., Zyuzel'skiy $\left(56.486^{\circ} \mathrm{N}\right.$ $\left.60.132^{\circ} \mathrm{E}\right), 2$.VII.1970, Zinov'ev ( 1 , ZISP); Ul'yanovsk Oblast: Shilovka $\left(54.052^{\circ} \mathrm{N} 48.645^{\circ} \mathrm{E}\right), 17 . \mathrm{VI} .2002$, V.B. Isaeva (1 $q$ ZMUM); Yulovo $\left(53.967^{\circ} \mathrm{N} 46.491^{\circ} \mathrm{E}\right), 16$.VIII.1995, V.B. Isaeva (1 $\sigma^{7}$, ZMUM); Chernaya Rechka $\left(54.177^{\circ} \mathrm{N} 49.75^{\circ} \mathrm{E}\right), 25 . \mathrm{VI} .2002$ V.B. Isaeva (1 $\sigma^{7}$, ZMUM); Vladimir Oblast: $11 \mathrm{~km}$ SW of Petushki (ca. $\left.55.860^{\circ} \mathrm{N} 39.577^{\circ} \mathrm{E}\right), 19$. VII.1992, Belokobyl'skiy (1 $\left.\mathrm{O}^{\top}, \mathrm{ZISP}\right)$; Volgograd Oblast: Kamyshin $\left(50.099^{\circ} \mathrm{N} 45.401^{\circ} \mathrm{E}\right)$, 16.VII.1950, G. Viktorov (2 $\Im^{\top} \odot^{7}$, ZMUM); Vorohezh Oblast: Usmanskiy Bor $\left(51.911^{\circ} \mathrm{N} 39.562^{\circ} \mathrm{E}\right), 24$. VIII.1966 and16.VIII.1970, Negrobov (4 $\overbrace{}^{7} \sigma^{7}, 2$ 우, ZISP); Varvarino $\left(51.209^{\circ} \mathrm{N} 41.719^{\circ} \mathrm{E}\right), 12$. VIII.1969, Dorovskaya ( $1 \sigma^{7}$, ZISP); Yaroslavl' Oblast: Berditsino $\left(57.455^{\circ} \mathrm{N}\right.$ 40.107E), 5.VI.1906, A. Yakovlev (1 9 , ZISP).

DIAGNOSTIC DESCRIPTION. Male. Female. Body-length 5.4-10.2 mm. Head. Frontal vitta black or dark brown, with whitish reflection; fronto-orbital plate black, greyish dusted. Face and parafacial white. Gena black. Postcranium black, grey dusted. 3 orbital and $3-$ 4 frontal setae present. Antenna black. Postpedicel approximately 2-2.5 times as long as wide, with 1-2 additional long setae external from plumose arista (Fig. 6). Arista plumose, the longest hairs approximately equal to the width of postpedicel. Palpus black. Thorax black, greyish dusted, only scutum mostly subshining. Acrostichals absent or as 2-3 hairs, dorsocentrals $3+3$ (including scapular seta), intra-alars $(0-1)+(1-2)$, supra-alars $1+2,2$ postpronotals. Scutellum with a pair of strong discal and a pair of strong apical setae. Legs subshining. Femora and tibiae of all legs black; tarsi of all legs brownish. Wing tinged with yellowish; veins brownish. Vein $\mathrm{R}_{1}$ setulose on apical third of dorsal surface. Abdomen black, delicately greyish dusted. Male sternite 4 rectangular, longer than wide. Sternite 5, epandrium and surstyli as in Figs 45-47.

DISTRIBUTION. Russia: Altai, Arkhangelsk Oblast, Bashkiria, Chelyabinsk Oblast, Karelia, Kemerov Oblast, Khakassia, Komi, Kursk Oblast, Leningrad Oblast, Moscow Oblast, Murmansk Oblast, Novosibirsk, Omsk Oblast, Pskov Oblast, Ryazan' Oblast, Smolensk Oblast, Sverdlovsk Oblast, Ul'yanovsk Ob- last, Vladimir Oblast, Volgograd Oblast, Vorohezh Oblast, Yaroslavl' Oblast. — Europe.

\section{Cordilura fulvifrons Ozerov, 1997}

Figs 48-50.

fulvifrons Ozerov, 1997: 1427 (Cordilura). Type-locality: Boitsovo $\left(47.02^{\circ} \mathrm{N} 134.21^{\circ} \mathrm{E}\right)$ (Russia, Khabarovsk Krai).

REMARK. This species was known from the typelocality only.

MATERIAL EXAMINED. Khabarovsk Krai: Boitsovo $20 \mathrm{~km}$ $\mathrm{N}$ of Bikin $\left(46.982^{\circ} \mathrm{N} 134.324^{\circ} \mathrm{E}\right), 250 \mathrm{~m}, 24-27 . V .1993, \mathrm{C}$. Lange \& J. Ziegler (1 paratype ${ }^{7}$, ZMUM); Primorsky Krai: Gribnoe, $10 \mathrm{~km} \mathrm{SE}$ of Chernigovka $\left(44.342^{\circ} \mathrm{N} 132.569^{\circ} \mathrm{E}\right), 21 . \mathrm{V} .1979$, A. Zinoviev (1 $\sigma^{7}$, ZISP).

DIAGNOSTIC DESCRIPTION. Male. Body-length 3.9-6.2 mm. Head. Frontal vitta yellow, matt; frontoorbital plate black, subshining. Face, parafacial and gena pale yellow. Postcranium subshining, black. 3 orbital and 3 frontal setae present. Antenna blackish. Postpedicel approximately 1.5 times as long as wide. Arista nearly bare. Palpus blackish. Thorax black, delicately greyish dusted, only scutum mostly subshining. Acrostichals absent, dorsocentrals 4+3 (including scapular seta), intra-alars absent, supra-alars $1+2,2$ postpronotals. Scutellum with a pair of strong discal and a pair of strong apical setae. Legs shining. Femora black, fore tibia and tarsi of all legs yellow, mid and hind tibiae yellowish dorsally and black ventrally. Wing tinged with yellowish; veins brownish. Vein $\mathrm{R}_{1}$ bare on apical third of dorsal surface. Abdomen black, delicately greyish dusted. Male sternite 4 trapezoid, wider than long. Sternite 5, epandrium and surstyli as in Figs 48-50.

Female unknown.

DISTRIBUTION. Russia: Khabarovsk Krai, Primorsky Krai.

\section{Cordilura fuscipes (Zetterstedt, 1838)} Figs 51-53.

fuscipes Zetterstedt, 1838: 726 (Cordylura). Type-locality: "Lapponia Tornensi ... et ... Stensele ... (Lappon, Suecica — Dalekarlia...)" (Sweden).

apicata Hendel, 1930: 4 (Cordylura). Type-locality: "Klutchi" (Kamchatka Krai, Russia).

nigrithorax Hendel, 1930: 5 (Cordylura). Type-locality: "Petropavlovsk" (Russia, Kamchatka Krai).

ochracea Hendel, 1930: 6 (Cordylura (Parallelomma)). Typelocality: "Petropavlovsk" (Russia, Kamchatka Krai).

REMARK. This species was recorded in Russia for European part without indicating specific locality by Gorodkov [1986: 16], for Murmansk Oblast by Engelmark [1999: 158, 159], for Far East by Hendel [1930: 4, 5, 6] and by Ozerov \& Krivosheina [2014: 207] (including Khabarovsk Krai).

MATERIAL EXAMINED. Altai: Lake Manzherok $\left(51.825^{\circ} \mathrm{N}\right.$ $85.812^{\circ}$ E), 10.V.2008, O. Kosterin (1 $\sigma^{\top}$, ZMUM); Seminsky Pass, River Turala, $\left(50.99^{\circ} \mathrm{N} 85.68^{\circ} \mathrm{E}\right), 1350 \mathrm{~m}, 8^{8}-12 . V I I .2016, \mathrm{~N}$. Vikhrev (1 + , ZMUM); Arkhangelsk Oblast: Nenetsky Nature Reserve, cordon "Bol'shoy Gusinets" $\left(68.175^{\circ} \mathrm{N}, 53.645^{\circ} \mathrm{E}\right)$, 910.VII.2008, N.E. Vikhrev, A.L. Ozerov (4 $\sigma^{\top} \sigma^{\top}, 4$ 우, ZMUM); the lower reaches of the River Pechora $\left(68.334^{\circ} \mathrm{N}, 53.304^{\circ} \mathrm{E}\right)$, 11.VII.2008, A.L. Ozerov (4 ○ొ $\sigma^{\top}, 2$ 우, ZMUM); Seyda env. $\left(67.064^{\circ} \mathrm{N}, 63.086^{\circ} \mathrm{E}\right), 28-31 . \mathrm{VII} .2011, \mathrm{~K}$. Tomkovich (3 $\sigma^{7} \sigma^{7}$, 
ZMUM); $73 \mathrm{~km} \mathrm{NW}$ of Nar'yan-Mar (ca. $67.981^{\circ} \mathrm{N} 51.608^{\circ} \mathrm{E}$ ), 3.VIII.1978, Gorodkov (1 $\mathrm{O}^{7}, 2$ 90 , ZISP); 70 km N of Nar'yanMar (ca. $\left.68.204^{\circ} \mathrm{N} 53.627^{\circ} \mathrm{E}\right), 18$.VIII.1978, Gorodkov (1 ${ }^{7}$, ZISP) Velikovisochnoe $\left(67.255^{\circ} \mathrm{N} 52.032^{\circ} \mathrm{E}\right), 18$.VIII.1978, Gorodkov (1 +, ZISP); Buryatia: Barguzinskiy Nature Reserve (ca. $54.35^{\circ} \mathrm{N}$ 109.51 $\left.{ }^{\circ} \mathrm{E}\right), 22$ and 23.VII.1965, Negrobov (2 $\sigma^{7} \sigma^{7}$, ZISP); Chukotka: bank of the River Anadyr $\left(64.72^{\circ} \mathrm{N} 175.21^{\circ} \mathrm{E}\right), 25$. VI.19.VII.2014, A. Barkalov ( $3 \bigcirc^{7} \sigma^{7}, 2$ 우, ZMUM and ISEA); Irkutsk Oblast: Chersky Peak $\left(51.528^{\circ} \mathrm{N} 103.587^{\circ} \mathrm{E}\right), 14$.VII.1984, Zlobin $\left(1 \bigcirc^{7}, 1\right.$,, ZISP); Kamchatka Krai: Valley of Geysers (ca. $54.43^{\circ} \mathrm{N}$ $\left.160.15^{\circ} \mathrm{E}\right)$, 12.VIII.1985, V. Zlobin (2 O9, ZISP); Apuka env. $\left(60.443^{\circ} \mathrm{N} 169.602^{\circ} \mathrm{E}\right)$, 7.VIII.1959, Gorodkov ( $2 \mathrm{O}^{7} \mathrm{\sigma}^{7}$, ZISP); Khakassia: W Sayan pass $\left(51.717^{\circ} \mathrm{N} 89.882^{\circ} \mathrm{E}\right), 2100 \mathrm{~m}, 9-12 . \mathrm{VII} .2017$ N. Vikhrev (3 $\sigma^{7} \sigma^{\top}, 1$, 1 ZMUM); Komi: Vorkuta $\left(67.5^{\circ} \mathrm{N} 64.0^{\circ} \mathrm{E}\right)$, 19-25.VII.2010, N. Vikhrev (5 $0^{7} \sigma^{7}, 10$ 우, ZMUM); Shchel'yabozh

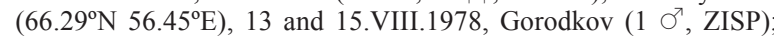
Krasnoyarsk Krai: Ergaki National Park $\left(52.84^{\circ} \mathrm{N} 93.25^{\circ} \mathrm{E}\right), 1450$ m, 27-29.VI.2017, N. Vikhrev (2 $\sigma^{7} \sigma^{\top}, 1$ \%, ZMUM); Magadan Oblast: Magadan env. $\left(59.6^{\circ} \mathrm{N} 150.8^{\circ} \mathrm{E}\right), 8$. VII.1978, V. Zherikhin (1 $\sigma^{7}$, ZMUM); Murmansk Oblast: Lake Vudyavr Basin (ca. $67.646^{\circ} \mathrm{N} 33.644^{\circ} \mathrm{E}$ ), 16.VII.1932, Fridolin (1 $\sigma^{7}$, ZISP); Primorsky Krai: Suputinsky [=Ussuriysky] Nature Reserve (ca. $43.635^{\circ} \mathrm{N}$ 132.311 ${ }^{\circ}$ E), 26.V.1962, A. Rasnitsyn, V. Sulimov (1 07, ZISP); Tyumen' Oblast: $\left(63.818^{\circ} \mathrm{N} 59.562^{\circ} \mathrm{E}\right), 1-8$. VII.2010, K. Tomko-

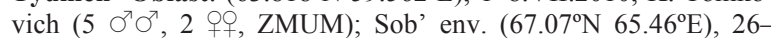
31.VII.2011, K. Tomkovich (3 $\sigma^{\top} \sigma^{7}$, ZMUM).

DIAGNOSTIC DESCRIPTION. Male. Female. Body-length 4.2-6.5 mm. Head. Frontal vitta yellow, matt; fronto-orbital plate yellow or black, delicately greyish dusted. Face yellowish, with whitish reflection. Parafacial and gena yellow. Postcranium yellow completely or black in upper part and yellow in lower third or quarter. 3 orbital and 2-3 frontal setae present. Scapus and pedicel yellow; postpedicel blackish, but yellowish basally, approximately 1.5 times as long as wide. Arista pubescent, the longest hairs approximately equal to $1 / 3-1 / 2$ width of postpedicel. Palpus yellow. Thorax from yellowish to black, delicately grey dusted. Acrostichals setulose in two rows, dorsocentrals $3+3$ (including scapular seta), intra-alars $(0-1)+(1-$ 2), supra-alars 1+2, 1-2 postpronotals. Scutellum with a pair of strong discal setae only; apical scutellar setae as setulae or absent. Legs shining, yellow completely, sometimes femora darkened dorsally. Wing tinged with brownish; veins blackish. Vein $\mathrm{R}_{1}$ bare on apical third of dorsal surface. Abdomen brownish or black, shining or delicately greyish dusted. Male sternite 4 rectangular, longer than wide. Male sternite 5, epandrium and surstyli as in Figs 51-53.

DISTRIBUTION. Russia: Altai, Arkhangelsk Oblast, Buryatia, Chukotka, Irkutsk Oblast, Kamchatka Krai, Khabarovsk Krai, Khakassia, Komi, Krasnoyarsk Krai, Magadan Oblast, Murmansk Oblast, Primorsky Krai, Tyumen' Oblast. - Europe, North America.

\section{Cordilura grunini Ozerov et Krivosheina, 2017 Figs 54-56.}

grunini Ozerov, Krivosheina, 2017: 2 (Cordilura). Type-locality: valley of the River Serebryanka (ca. $45.048^{\circ} \mathrm{N} 136.635^{\circ} \mathrm{E}$ ) (Russia, Primorsky Krai).

REMARK. This species was known from the typelocality only.

MATERIAL EXAMINED. Primorsky Krai: Sikhote-Alin Nature Reserve, Blagodatnoe $\left(44.975^{\circ} \mathrm{N} 136.526^{\circ} \mathrm{E}\right), 1-6 . V I .2018$,
M. Sergeev (1 $\sigma^{7}$, ZMUM); Kedrovaya Pad' Nature Reserve (43.104 $\left.{ }^{\circ} \mathrm{N} 131.512^{\circ} \mathrm{E}\right), 22 . V .1983$, Zlobin (1 $\left.\sigma^{7}, \mathrm{ZISP}\right)$.

DIAGNOSTIC DESCRIPTION. Male. Body-length $4.6 \mathrm{~mm}$. Head. Frontal vitta brownish, with whitish reflection; fronto-orbital plate black. Face and parafacial whitish. Gena yellow, whitish dusted. Postcranium black, subshining. 2 orbital and 2-3 frontal setae present. Antenna black. Postpedicel approximately 1.5 times as long as wide. Arista black, pubescent in basal quarter (the longest hairs not longer than greatest diameter of arista) and the rest bare. Palpus blackish. Thorax black, scutum subshining, thoracic sclerites greyish dusted. Acrostichals setulose in two irregular rows, dorsocentrals $3+3$ (including short scapular seta), intra-alars absent, supra-alars 1+2, 2 postpronotals. Scutellum with a pair of strong discal and a pair of strong apical setae. Legs. Ground-colour black; all coxae and femora black, mid and hind tibiae blackish; fore tibia and all tarsi yellow. Wing tinged with brownish; veins brown. Vein $\mathrm{R}_{1}$ bare on dorsal surface. Abdomen black, thinly greyish dusted. Male sternite 4 approximately 2 times as wide as long. Male sternite 5, epandrium and surstyli as in Figs 54-56.

Female unknown.

DISTRIBUTION. Russia: Primorsky Krai.

\section{Cordilura impudica (Rondani, 1867)} Figs 19, 57-59.

impudica Rondani, 1867: 97 (Cordylura). Type-locality: “in agri parmensis planitie..." (vicinity of Parma, Italy).

umbrosa Loew, 1873: 246 (Cordylura). Type-locality: "Losoncz" [Luèenec, $48.333^{\circ} \mathrm{N} 19.666^{\circ} \mathrm{E}$ ] (Slovakia).

REMARKS. Noted by Gorodkov [1970: 448, 1986 : 15, as C. umbrosa] for south of European part of Russia without indicating specific locality. Recorded by Ovchinnikov [2004: 422] from Saratov Oblast and Astrakhan' Oblast.

MATERIAL EXAMINED. Altai: Lake Kolyvanskoe $\left(51.363^{\circ} \mathrm{N}\right.$ 82.191 $\left.{ }^{\circ} \mathrm{E}\right)$, 8.IX.2007, O. Kosterin (1 $\sigma^{7}$, ZMUM); Astrakhan' Oblast: Zyuzino $\left(45.751^{\circ} \mathrm{N} 47.678^{\circ} \mathrm{E}\right), 8-9 . V .2010$, K. Tomkovich (1 $\sigma^{7}$, ZMUM); Astrakhan' Nature Reserve (ca. $\left.46.110^{\circ} \mathrm{N} 48.749^{\circ} \mathrm{E}\right)$, 14, 16.V. and 7.VIII.1963, Pisarev (1 フ 3 우, ZISP); Bashkiria: Beloretsk $\left(54.051^{\circ} \mathrm{N} 58.268^{\circ} \mathrm{E}\right), 12 . \mathrm{VIII} .2012$, D. Gavryushin (1 $\mathrm{O}^{7}$, ZMUM); Kursk Oblast: Oboyan' $\left(51.191^{\circ} \mathrm{N} 36.312^{\circ} \mathrm{E}\right), 25-$ 26.V., 20-21.VII.2007, A.L. Ozerov (2 우, ZMUM); Zorino $\left(51.172^{\circ} \mathrm{N} 36.353^{\circ} \mathrm{E}\right), 14 . \mathrm{V} .2008, \mathrm{~K}$. Tomkovich $\left(3 \mathrm{O}^{7} \sigma^{7}, 1\right.$, ZMUM); Mordovia: Mordovsky Nature Reserve, Lake Inoskoe $\left(54.728^{\circ} \mathrm{N}, 43.15^{\circ} \mathrm{E}\right), 20 . V .2020$, N. Vikhrev (1 $\sigma^{\top}$, ZMUM); Moscow Oblast: Lugovaya $\left(56.203^{\circ} \mathrm{N} 37.823^{\circ} \mathrm{E}\right), 26$. VI.1993, A.L.

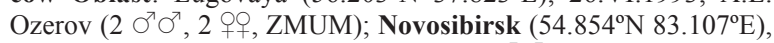
11.V.2008, 9-10.IX.2009, O. Kosterin (2 $\sigma^{7} \sigma^{7}$, ZMUM); Primorsky Krai: Novokievsk[=Kraskino] $\left(42.713^{\circ} \mathrm{N} 130.781^{\circ} \mathrm{E}\right)$, 8.VI.1933, A. Mishchenko ( 2 ㅇ, ZISP); Sakhalin Oblast: Sakhalin I., Moskalevo (53.567º $\left.142.504^{\circ} \mathrm{E}\right)$, 13.VIII.2001, A. Leley (1 , ZMUM); Saratov Oblast: $5 \mathrm{~km} \mathrm{~S}$ of N. Bannovka $\left(50.666^{\circ} \mathrm{N}\right.$, $\left.45.633^{\circ} \mathrm{E}\right), 11$. VII.2005, Nartshuk ( $\sigma^{\top}, 1$ ․, ZISP); Nizhnaya Bannovka $\left(50.666^{\circ} \mathrm{N}, 45.633^{\circ} \mathrm{E}\right), 4-5 . \mathrm{VII} .2003$, A. Ovchinnikov (9 $\Im^{\top} \Im^{7}, 14$ 우, ZISP); Zabaykalsky Krai: $20 \mathrm{~km}$ E of Dauriya station $\left(49.931^{\circ} \mathrm{N} 116.851^{\circ} \mathrm{E}\right), 6 . \mathrm{VI} .1943$, Dubinin (1 $\circ$, ZISP).

ADDITIONAL MATERIAL EXAMINED. Uzbekistan: Tashkent $\left(41.313^{\circ} \mathrm{N} 69.286^{\circ} \mathrm{E}\right), 2$. IV.1929, Rohdendorf (1 $\left.{ }^{\circ}, \mathrm{ZISP}\right)$.

DIAGNOSTIC DESCRIPTION. Male. Female. Body-length 6.0-7.6 mm. Head. Frontal vitta from brown to black, with whitish reflection; fronto-orbital 


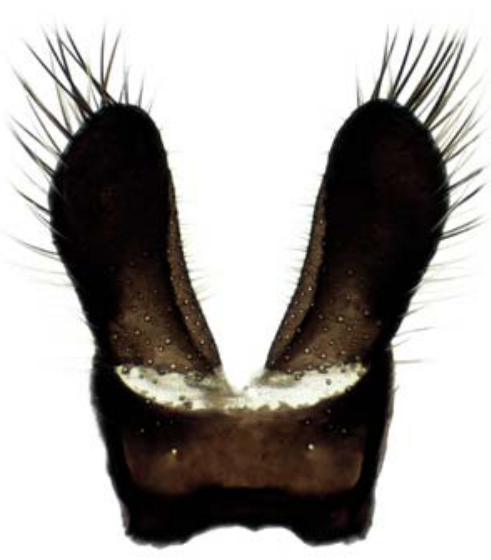

60

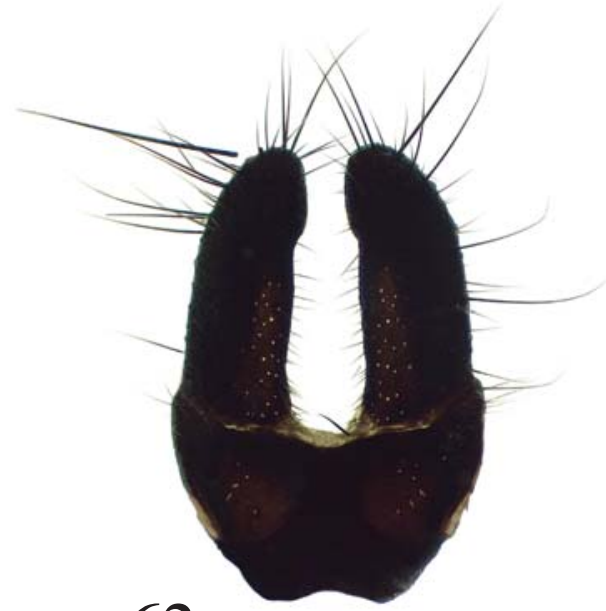

63

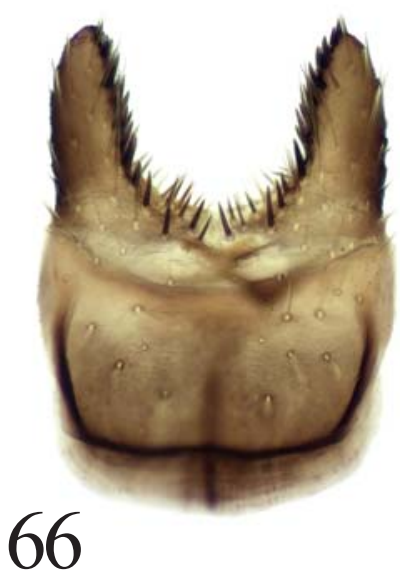

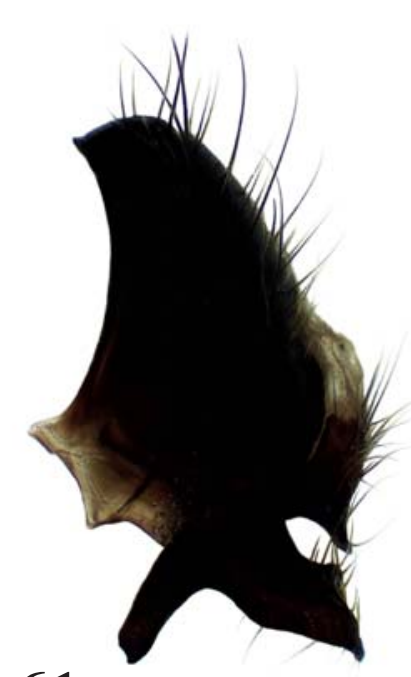

61
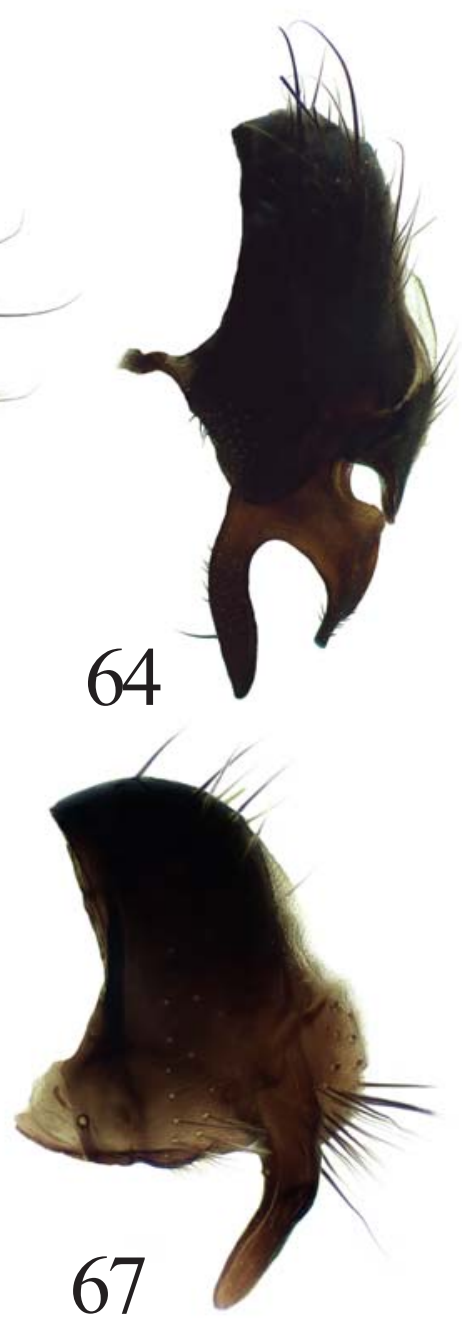
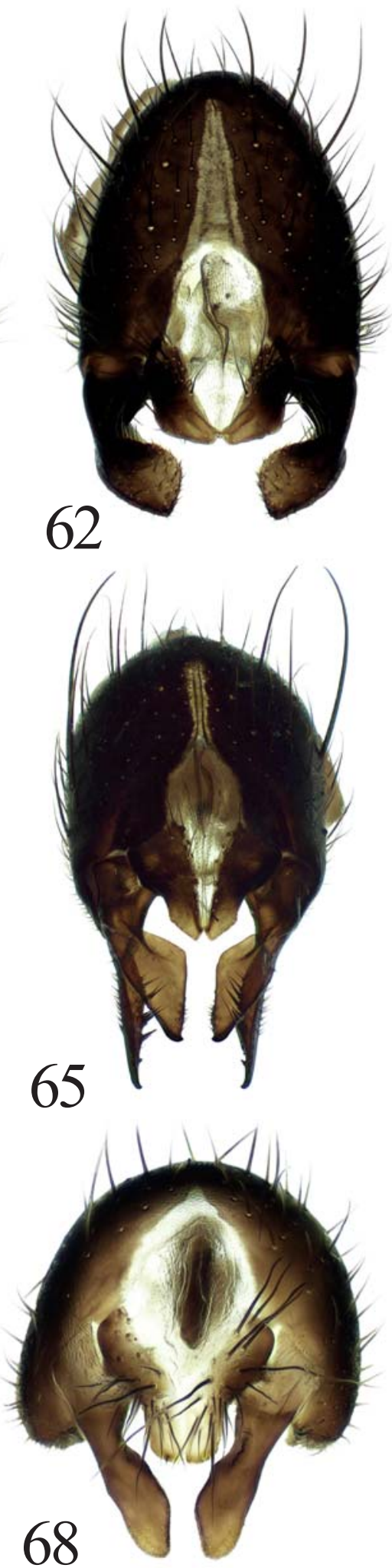

Figs 60-68. Cordilura kakaberrans Ozerov (60-62), C. kosterini Ozerov et Krivosheina (63-65) and C. krocha Ozerov (66-68): 60, 63, 66 - male sternite 5; 61, 64, 67 - epandrium and surstyli, lateral view; 62, 65, 68 — same, dorsal view. 63-65 — after Ozerov \& Krivosheina, 2014, figs 10-12.

Рис. 60-68. Cordilura kakaberrans Ozerov (60-62), C. kosterini Ozerov et Krivosheina (63-65) и C. krocha Ozerov (66-68): 60, 63, 66 - стернит 5 самца; 61, 64, 67 - эпандрий и сурстили, сбоку; 62, 65, 68 - то же, cверху. 63-65 - по Ozerov \& Krivosheina, 2014, figs $10-12$. 
plate black, greyish dusted. Face and parafacial yellowish with whitish reflection. Gena blackish. Postcranium black, grey dusted. 3 orbital and 2-3 frontal setae present. Antenna black. Postpedicel approximately 2 times as long as wide. Arista plumose, the longest hairs approximately equal to the width of postpedicel. Palpus black. Thorax black, delicately grey dusted. Acrostichals setulose in two rows, dorsocentrals $3+3$ (including scapular seta), intra-alars $(0-1)+2$, supra-alars $1+2,2$ postpronotals. Scutellum with a pair of strong discal setae only; apical scutellar setae as setulae or absent. Legs greyish dusted. Fore coxa black. Femora of all legs black. Tibiae and tarsi of all legs yellow. Wing tinged with brownish; veins blackish. Vein $\mathrm{R}_{1}$ setulose on apical third of dorsal surface (Fig. 19). Abdomen black, delicately grey dusted. Male sternite 4 rectangular, longer than wide. Male sternite 5, epandrium and surstyli as in Figs 57-59.

Description, figures and photo of female ovipositor is given by Ovchinnikov [2009: 624-625, 630, as Cordilura umbrosa].

DISTRIBUTION. Russia: Altai, Bashkiria, Kursk Oblast, Mordovia, Moscow Oblast, Novosibirsk Oblast, Primorsky Krai, Sakhalin Oblast, Saratov Oblast, Zabaykalsky Krai. - Europe, Kazakhstan [Gorodkov, 1986: 15], Uzbekistan (first record).

\section{Cordilura kakaberrans Ozerov, 1997 Figs 60-62.}

kakaberrans Ozerov, 1997: 1424 (Cordilura). Type-locality: 40 km SE of Ussuriysk (Russia, Primorsky Krai).

REMARK. Recorded in Russia from Khabarovsk Krai and Primorsky Krai [Ozerov, Krivosheina, 2014: 207].

MATERIAL EXAMINED. Primorsky Krai: Kamenushka (43.634 $\left.{ }^{\circ} \mathrm{N} 132.222^{\circ} \mathrm{E}\right), 29$. VII.1983,7.VI.1985, A.L. Ozerov (3 $\sigma^{7} \sigma^{7}$, 3 우, ZMUM); Ryazanovka (42.797 $\left.\mathrm{N} 131.244^{\circ} \mathrm{E}\right), 10 . \mathrm{VI} .1989$, A. Shatalkin (1 $\sigma^{7}$, ZMUM); Udegeyskaya Legenda National Park $\left(45.766^{\circ} \mathrm{N} 135.465^{\circ} \mathrm{E}\right), 23$.VII.2009, A. Ovchinnikov (1 9 , ZISP).

DIAGNOSTIC DESCRIPTION. Male. Female. Body-length 5.2-7.4 mm. Head. Frontal vitta from reddish yellow to blackish, with whitish reflection; frontoorbital plate black, greyish dusted. Face and parafacial yellowish with whitish reflection. Gena yellow or brownish. Postcranium black, grey dusted. 2 orbital and 2-3 frontal setae present. Scapus and pedicel brownish; postpedicel blackish, approximately 2 times as long as wide. Arista plumose, the longest hairs approximately equal to the width of postpedicel. Palpus black or dark brown. Thorax black, grey dusted. Acrostichals setulose in two rows, dorsocentrals $3+3$ (including scapular seta), intra-alars absent, supra-alars $(0-1)+2,2$ postpronotals. Scutellum with a pair of strong discal setae and a pair of apical scutellar setulae. Legs greyish dusted. Fore coxa black; femora black; tibia and tarsus of fore leg yellow; tibiae and tarsi of mid and hind legs from brownish to black. Wing tinged with brownish; veins blackish. Vein $\mathrm{R}_{1}$ bare on apical third of dorsal surface. Abdomen black, delicately grey dusted. Male sternite 4 rectangular, longer than wide. Male sternite 5 , epandrium and surstyli as in Figs 60-62.
DISTRIBUTION. Russia: Khabarovsk Krai, Primorsky Krai.

\section{Cordilura kosterini Ozerov et Krivosheina, 2014} Figs 63-65.

kosterini Ozerov, Krivosheina, 2014: 207 (Cordilura). Typelocality: Lotos Lake $\left(42.46^{\circ} \mathrm{N} 130.64^{\circ} \mathrm{E}\right)$ (Russia, Primorsky Krai).

REMARKS. This species is known from the typelocality only. A single male was collected on leaves of Nelumbo nucifera Gaertner, 1788 (Nelumbonaceae).

DIAGNOSTIC DESCRIPTION. Male. Bodylength $6.6 \mathrm{~mm}$. Head. Frontal vitta black, matt; fronto-orbital plate black, subshining. Face and parafacial yellow with whitish reflection. Gena blackish. Postcranium black, greyish dusted. 3 orbital and 3 frontal setae present. Antenna black. Postpedicel approximately 2 times as long as wide. Arista pubescent, the longest hairs approximately equal to $1 / 2$ width of postpedicel. Palpus black. Thorax black, greyish dusted. Acrostichals absent, dorsocentrals 3+3 (including scapular seta), intra-alars $1+2$, supra-alars $1+2,2$ postpronotals. Scutellum with a pair of strong discal setae. Legs greyish dusted. Femora of all legs black; tibiae and tarsi of all legs yellow, only hind tibia darkened dorsally. Wing tinged with brownish; veins brownish. Vein $\mathrm{R}_{1}$ setulose on apical third of dorsal surface. Abdomen black, grey dusted. Male sternite 4 rectangular, longer than wide. Sternite 5, epandrium and surstyli as in Figs 63-65.

Female unknown.

DISTRIBUTION. Russia: Primorsky Krai.

Cordilura krocha Ozerov, 2007 Figs 66-68.

krocha Ozerov, 2007b: 123 (Cordilura). Type-locality: Zeya Town (Russia, Amur Oblast).

REMARK. The species was recorded in Russia from Amur Oblast only.

MATERIAL EXAMINED. Amur Oblast: Zeya Town $\left(53.748^{\circ} \mathrm{N} 127.261^{\circ} \mathrm{E}\right), 3$ and 17.VIII.1982, A. Shatalkin $\left(2 \bigcirc^{\top} \sigma^{\top}\right.$, ZMUM); Zeysky Nature Reserve, cordon "52 km" $\left(54.087^{\circ} \mathrm{N}\right.$ $\left.126.871^{\circ} \mathrm{E}\right), 13-14$.VIII.1981, A. Ozerov, O. Gorbunov, A. Shatalkin

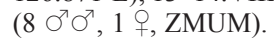

DIAGNOSTIC DESCRIPTION. Male, female. Body-length 3.6-4.0 mm. Head. Frontal vitta black or blackish in upper half and reddish-yellow in lower half; fronto-orbital plate blackish, greyish dusted. Face, parafacial and gena yellowish white. Postcranium blackish in upper half and yellow-whitish in lower half, greyish dusted. 2 orbital and 2 frontal setae present. Antenna yellow, postpedicel darkened in lower half. Postpedicel approximately 2 times as long as wide. Arista nearly bare. Palpus pale yellow. Thorax greyish dusted; completely yellow in female; scutum and mediotergite black, pleural sclerites dark, but lighter in lower part in male. Acrostichals setulose in two rows or absent, dorsocentrals 3+3 (including scapular seta), intra-alars $(0-1)+0$, supra-alars $1+2,1$ strong postpronotal. Scutellum with a pair of strong discal setae only. 
Legs yellow; tarsomere 5 of hind leg dark brownish in male; all tarsi yellow in female. Wing tinged with light brownish; veins brownish. Vein $\mathrm{R}_{1}$ bare on apical third of dorsal surface. Abdomen blackish greyish dusted; tergites 5 and 6 shining in female. Male sternite 4 rectangular, longer than wide. Male sternite 5, epandrium and surstyli as in Figs 66-68.

DISTRIBUTION. Russia: Amur Oblast.

Cordilura monochroma Ozerov et Krivosheina, 2014 Figs 10, 69-71.

monochroma Ozerov, Krivosheina, 2014: 209 (Cordilura). Type-locality: Yuzhno-Kurilsk (43.026ㅇ $\left.145.862^{\circ} \mathrm{E}\right)$ (Russia, Sakhalin Oblast).

REMARK. This species was described on material from Sakhalin Oblast and Primorsky Krai [Ozerov, Krivosheina, 2014: 209].

MATERIAL EXAMINED. Amur Oblast: Klimoutsy $\left(51.470^{\circ} \mathrm{N}\right.$ $\left.127.599^{\circ} \mathrm{E}\right), 22$ and 30.V.1958, 31.V. and 17.VI.1959, Zinov'ev (3 $\sigma^{\top} \sigma^{\top}, 1$ O ZISP); Buryatia: Selenduma $\left(50.916^{\circ} \mathrm{N} 106.239^{\circ} \mathrm{E}\right)$, 25.VI.1971, V. Richter (3 $\sigma^{\top} \sigma^{7}, 1$, ZISP); Khabarovsk Krai: Lake Sindinskoe $\left(48.92^{\circ} \mathrm{N} 136.24^{\circ} \mathrm{E}\right), 7 . V I .2014$, N. Vikhrev (2 오, ZMUM); Primorsky Krai: Terney $\left(45.052^{\circ} \mathrm{N} 136.609^{\circ} \mathrm{E}\right)$ 26.VI.1937, Grunin (1 $\sigma^{7}$, ZISP); Khasan $\left(42.43^{\circ} \mathrm{N} 130.647^{\circ} \mathrm{E}\right)$ 25.V.1979, A. Zinov'ev (1 フ7, ZISP); same place, 12.VII.1962, Nartshuk (1 + , ZISP); Sikhote-Alin Nature Reserve, Blagodatnoe $\left(44.975^{\circ} \mathrm{N} 136.526^{\circ} \mathrm{E}\right), 1-6 . V I .2018$, M. Sergeev (1 $\sigma^{7}, \mathrm{ZMUM}^{\circ}$; $40 \mathrm{~km} \mathrm{SE}$ of Ussuriysk, Ussuriysky Nature Reserve $\left(43.634^{\circ} \mathrm{N}\right.$ 132. $\left.222^{\circ} \mathrm{E}\right), 26 . V .1985$, A. Ozerov (1 ㅇ, ZMUM); Santakheza [now=Novosel'skoe] $\left(44.773^{\circ} \mathrm{N} 132.698^{\circ} \mathrm{E}\right), 6 . V I .1971$, Pinskaya (1 ${ }^{7}$, ZISP); Sakhalin Oblast: Kunashir I., Kurilskiy Nature Reserve, cordon Andreevskiy $\left(43.54^{\circ} \mathrm{N} 145.37^{\circ} \mathrm{E}\right), 6-8$. VII.2014, I.A. Gomyranov (1 $\sigma^{7}$, ZMUM); Kunashir I., Alekhino $\left(43.955^{\circ} \mathrm{N}\right.$ $\left.145.5927^{\circ} \mathrm{E}\right)$, 15.VI.1973, Kerzhner (1 $\sigma^{7}$, ZISP); Kunashir I., Sernovodsk $\left(43.912^{\circ} \mathrm{N} 145.641^{\circ} \mathrm{E}\right), 30 . \mathrm{VI} .1968$, Nartshuk $\left(2 \sigma^{\top} \sigma^{\top}, 1\right.$ +, ZISP); same place, 6.VII.1973, Kerzhner (1 $\sigma^{7}$, ZISP); same place, 6.VII.1973, V. Richter (8 Oフ $\sigma^{\top}, 5$ 우, ZISP).

DIAGNOSTIC DESCRIPTION. Male. Female. Body-length 6.3-8.1 mm. Head. Frontal vitta reddish, with silver reflection; fronto-orbital plate black, whitish dusted. Face and parafacial yellow, with silver reflection. Gena from yellow to black. Postcranium black, whitish dusted. 3 orbital and 3-4 frontal setae present. Antenna black. Postpedicel approximately 2 times as long as wide. Arista plumose, the longest hairs approximately equal to the width of postpedicel. Palpus black. Thorax black, whitish dusted. Acrostichals setulose usually in two rows (1-3 pairs of presutural and $0-1$ pair of postsutural (prescutellar)), dorsocentrals $3+3$ (including scapular seta), intra-alars $1+2$, supra-alars $1+2,2$ postpronotals. Scutellum with a pair of strong discal and a pair of strong apical setae. Legs. Coxae and fore femora of all legs black; fore and mid tibiae yellow, hind tibia from yellow to black; tarsi of all legs brownish or blackish, ventral surface of fore tarsus uniformly brownish (Fig. 14). Wing tinged with brownish; veins brown. Vein $\mathrm{R}_{1}$ bare on apical third of dorsal surface. Abdomen black in ground colour. Tergites greyish dusted. Male sternite 4 trapezoid, longer than wide. Male sternite 5, epandrium and surstyli as in Figs 69-71.

DISTRIBUTION. Russia: Amur Oblast, Buryatia, Khabarovsk Krai, Primorsky Krai, Sakhalin Oblast.
Cordilura nartshukae Ozerov et Krivosheina, 2015 Fig. 18.

nartshukae Ozerov, Krivosheina, 2015: 489 (Cordilura). Typelocality: riverhead of the River Chapingou [ $=$ Krounovka], tributary of the River Shufan [=Borisovka] (ca. $43.615^{\circ} \mathrm{N} 131.541^{\circ} \mathrm{E}$ ) (Russia, Primorsky Krai).

REMARK. The species is known from the typelocality only.

DIAGNOSTIC DESCRIPTION. Female. Bodylength $7.2 \mathrm{~mm}$. Head. Frontal vitta and fronto-orbital plate yellow, whitish dusted. Face and parafacial yellow with whitish reflections. Gena and postcranium yellow. 1-2 orbital and 4-5 frontal setae present. Antenna yellow. Postpedicel approximately 2.5 times as long as wide. Arista yellow, pubescent, the longest hairs approximately equal to $1 / 3-1 / 2$ width of postpedicel. Palpus yellow. Thorax yellow, thinly greyish dusted. Acrostichals setulose in two irregular rows, dorsocentrals $3+3$ (including scapular seta), intra-alars $0+1$, supra-alars $1+2,2$ postpronotals. Scutellum with a pair of strong discal setae and a pair of apical setulae. Legs completely yellow. Wing (Fig. 18) tinged with brownish; veins pale brown. Vein $\mathrm{R}_{2+3}$ with a brown spot apically. Intersection of vein $\mathrm{M}$ and crossvein dmcu with a brown spot. Apex of wing darkened. Vein $\mathrm{R}_{1}$ setulose on apical third of dorsal surface. Abdomen brownish, thinly greyish dusted.

Male unknown.

DISTRIBUTION. Russia: Primorsky Krai.

Cordilura negrobovi Ozerov et Krivosheina, 2017 Figs 72-74.

negrobovi Ozerov, Krivosheina, 2017: 4 (Cordilura). Typelocality: Tagarhay $\left(51.878^{\circ} \mathrm{N} 102.392^{\circ} \mathrm{E}\right)$ (Russia, Buryatia).

REMARK. This species is known from the typelocality only.

DIAGNOSTIC DESCRIPTION. Male. Body-length $6.7 \mathrm{~mm}$. Head. Frontal vitta black, in upper third brownish; fronto-orbital plate black, whitish dusted. Face and parafacial white. Gena and postgena brownish. Postcranium black. 2 orbital and 4 frontal setae present. Antenna brownish. Postpedicel approximately 2 times as long as wide. Arista yellow, pubescent or plumose, the longest hairs approximately equal to $1 / 2-3 / 4$ width of postpedicel. Palpus yellow. Thorax black. Scutum and scutellum shining; anepisternum shining, but along anterior margin greyish dusted; anepimeron shining completely; mediotergite shining along border with abdomen; the rest parts of thorax greyish dusted. Acrostichals absent, dorsocentrals 3+3 (including scapular seta), intra-alars absent, supra-alars $0+2,1$ postpronotal. Scutellum with a pair of strong discal setae. Legs. Fore coxa yellow, mid and hind coxae blackish. Femora of all legs black. Fore tibia and tarsi of all legs yellow. Mid and hind tibiae brownish. Wing tinged with brownish; veins brown. Vein $\mathrm{R}_{1}$ bare on dorsal surface. Abdomen black, thinly greyish dusted. Male sternite 4 approximately 2 times as long as wide. Sternite 5 with long wide lobes (Fig. 72). Surstyli bifurcate (Figs 73, 74). 

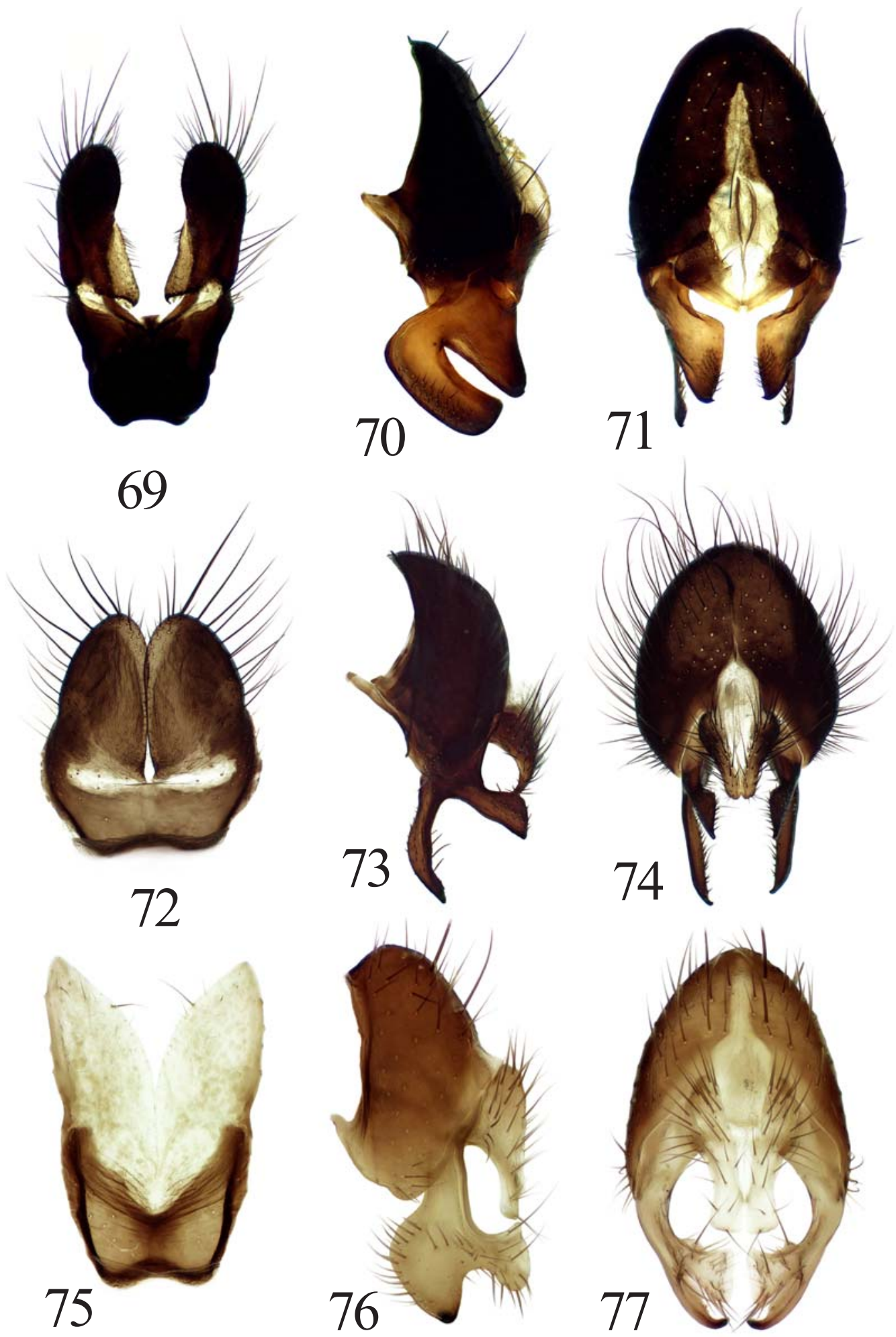

Figs 69-77. Cordilura monochroma Ozerov et Krivosheina (69-71), C. negrobovi Ozerov et Krivosheina (72-74) and C. nubecula Sasakawa (75-77): 69, 72, 75 — male sternite 5; 70, 73, 76 - epandrium and surstyli, lateral view; 71, 74, 77 — same, dorsal view. 69 71 - after Ozerov \& Krivosheina, 2014, figs 16, 18, 19; 72-74 - after Ozerov \& Krivosheina, 2017, figs 7-9; 75-77 — after Ozerov \& Krivosheina, 2012b, figs 5, 7, 8 .

Pис. 69-77. Cordilura monochroma Ozerov et Krivosheina (69-71), C. negrobovi Ozerov et Krivosheina (72-74) и C. nubecula Sasakawa (75-77): 69, 72, 75 - стернит 5 самца; 70, 73, 76 - эпандрий и сурстили, сбоку; 71, 74, 77 - то же, сверху. 69-71 - по Ozerov \& Krivosheina, 2014, figs 16, 18, 19; 72-74 — по Ozerov \& Krivosheina, 2017, figs 7-9; 75-77 — по Ozerov \& Krivosheina, $2012 \mathrm{~b}$, figs $5,7,8$. 
Female unknown.

DISTRIBUTION. Russia: Buryatia.

\section{Cordilura nubecula Sasakawa, 1986}

Figs 75-77.

nubecula Sasakawa, 1986: 43 (Cordilura). Type-locality: Utukusigahara (Japan, Nagano Pref.).

REMARK. The species was firstly registered in Russia by Ozerov \& Krivosheina [2012b: 4] from Khabarovsk Krai, and after also included in list of Cordilura species of Far East of Russia [Ozerov, Krivosheina, 2014: 211].

MATERIAL EXAMINED. Khabarovsk Krai: Boitsovo 20 $\mathrm{km} \mathrm{N}$ of Bikin $\left(46.982^{\circ} \mathrm{N} 134.324^{\circ} \mathrm{E}\right), 250 \mathrm{~m}, 24-27 . V .1993, \mathrm{C}$. Lange \& J. Ziegler ( $10^{7}$, ZMUM).

DIAGNOSTIC DESCRIPTION. Male. Body-length 6.2-7.3 mm [Sasakawa, 1986: 44; Iwasa, 2020: 477]. Head. Frontal vitta and fronto-orbital plate yellow. Face, parafacial and gena yellowish, with weak whitish reflection. Postcranium yellow in lower part and black in upper half. 2 orbital and 2-3 frontal setae present. Antenna pale yellow. Postpedicel approximately 2 times as long as wide. Arista plumose, the longest hairs approximately equal to the width of postpedicel. Palpus yellow. Thorax yellow in ground colour, but scutum black completely, anepimeron, katatergite and mediotergite blackish. Acrostichals setulose in two rows, dorsocentrals $3+3$ (including scapular seta), intra-alars $1+(0-1)$, supra-alars $1+2,2$ postpronotals. Scutellum with a pair of strong discal setae only; apical scutellar setae as setulae. Legs shining, yellow. Wing tinged with brownish, with a distinct brown spot apically; veins blackish. Vein $\mathrm{R}_{1}$ setulose on apical third of dorsal surface. Abdomen black, subshining. Sternite 4 trapezoid, slightly longer than wide. Sternite 5, epandrium and surstyli as in Figs 75-77.

Female. Not studied.

DISTRIBUTION. Russia: Khabarovsk Krai. — Japan [Sasakawa, 1986: 43; Iwasa, 2020: 477].

\section{Cordilura nubilosa Ozerov et Krivosheina, sp.n.} Figs 78-80.

MATERIAL EXAMINED. Holotype $\sigma^{\top}$, Russia: Primorsky Krai: Sikhote-Alin', the top of the Mt. Oblachnaya $\left(43.695^{\circ} \mathrm{N}\right.$ 134.199 ${ }^{\circ}$ E), 3.VII.1962, Kovalev (ZISP).

DESCRIPTION. Male. Length of body $4.2 \mathrm{~mm}$. Length of wing $4.8 \mathrm{~mm}$.

Head. Frontal vitta and fronto-orbital plate reddish, thinly whitish dusted. Ocellar triangle black. Face, parafacial and gena yellow with whitish reflections. Postcranium black in upper part and yellow in lower third. Setae: 2 orbitals, 2 frontals, 1 ocellar, 1 long inner vertical, 1 outer vertical (approximately 0.5 times as long as inner vertical), 1 postocellar; 1 pair of strong vibrissae present. Scapus and pedicel yellowish. Postpedicel blackish, rounded apically, approximately 2.5 times as long as wide. Arista nearly bare, longest hairs not exceeding greatest diameter of arista. Palpus yellow with a thin apical seta.
Thorax thinly greyish dusted, blackish, only katepisternum and meron yellow. Acrostichals setulose in two rows, dorsocentrals $3+1$ (including scapular seta), intra-alars $0+1$, supra-alars $1+2,1$ postpronotal, 2 notopleurals, 2 postalars. Proepisternum covered with fine hairs and with 1 seta near ventral margin. Proepimeron with 1-2 setae ventral to spiracle. Anepisternum with a few hairs in posterior half and 2 black setae near posterior margin. Katepisternum with yellow hairs in ventral corner and 1 strong long black seta in posterodorsal corner. Anepimeron bare. Scutellum with a pair of strong discal setae only; apical scutellar setae as setulae.

Legs completely yellow. Fore femur with rows of dorsal and posteroventral setae. Fore tibia with 2 posterodorsal, 2 anterodorsal, 2 posteroventral, 1 dorsal preapical and 1 posterior apical setae. Mid femur with a row of anterior setae and with 1 posterior apical seta. Mid tibia with 2 anterodorsal, 2 posterodorsal, 1 anteroventral setae, and with a ring of apicals. Hind femur with a row of anterodorsal setae and 2 anteroventral setae in apical third. Hind tibia with 1 posterodorsal, 2 anterodorsal, 1 anteroventral, 1 dorsal preapical, and 1 apical anteroventral setae.

Wing tinged with yellowish; veins pale brown. Vein $\mathrm{R}_{1}$ setulose on apical third of dorsal surface. Calypters, margins of calypters, and halteres yellowish.

Abdomen blackish, thinly greyish dusted. Syntergite 1+2 with 3-4 lateral discal and with a row of marginal setae; tergites 3-6 each with a row of marginal setae. Sternite 4 rectangular, approximately as long as wide. Sternite 5, epandrium and surstyli as in Figs 78-80.

Female unknown.

COMPARISON. New species is closely related to East Palaearctic species C. amurensis Ozerov, 2007, but is distinguished from this species by colour of scutum and structure of surstyli. C. amurensis has scutum of thorax reddish yellow and longer surstyli (Fig. 38), undoubtedly curved in median part (Fig. 37). New species has scutum of thorax blackish and surstyli shorter and straight (Figs 79, 80).

DISTRIBUTION. Russia: Primorsky Krai.

\section{Cordilura picipes (Meigen, 1826)} Figs 2, 81-83.

picipes Meigen, 1826: 232 (Cordylura). Type-locality: not given.

biseta Loew, 1864: 21 (Cordylura). Type-localities: "Dänemark, ganz Deutschland" (Denmark, Germany).

REMARKS. Recorded by Gorodkov [1986: 14] for Leningard Oblast and for central and south parts of European Russia, but without indicating specific locality. Sifner [2008: 120] recorded this species from Moscow Oblast, Humala \& Polevoi [2009: 71] from Karelia and Ovchinnikov [2004: 421] from Yaroslavl' Oblast.

MATERIAL EXAMINED. Altay: Artybash env, $\left(51.796^{\circ} \mathrm{N}\right.$ 87.265 ${ }^{\circ}$ ) , 16-18.VII.2006, Nartshuk ( 2 +क, ZISP); Karelia: Kizhi

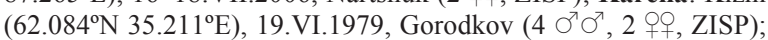
Kursk Oblast: Oboyan' $\left(51.191^{\circ} \mathrm{N} 36.312^{\circ} \mathrm{E}\right), 26 . \mathrm{V} .2007, \mathrm{~N}$. 


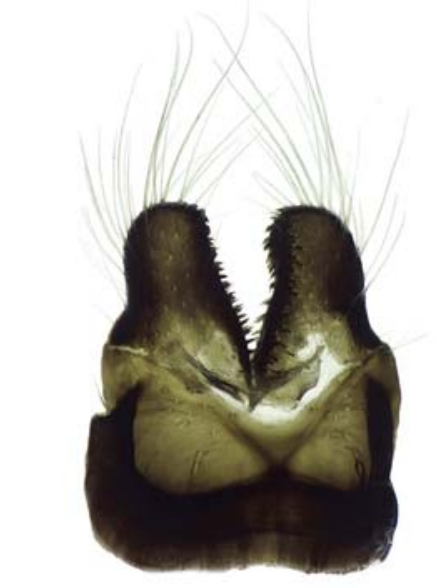

78

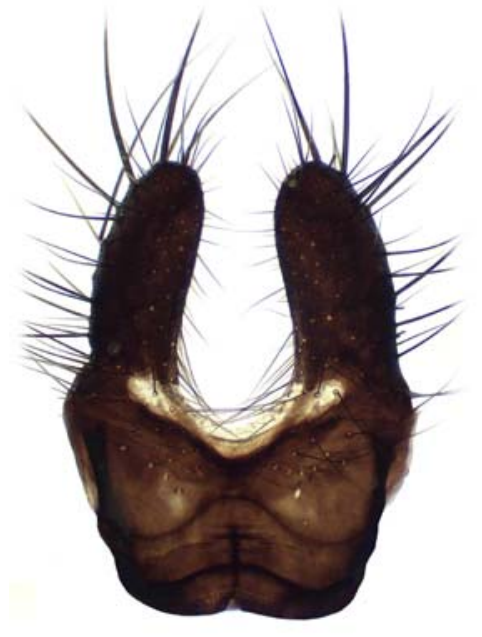

81

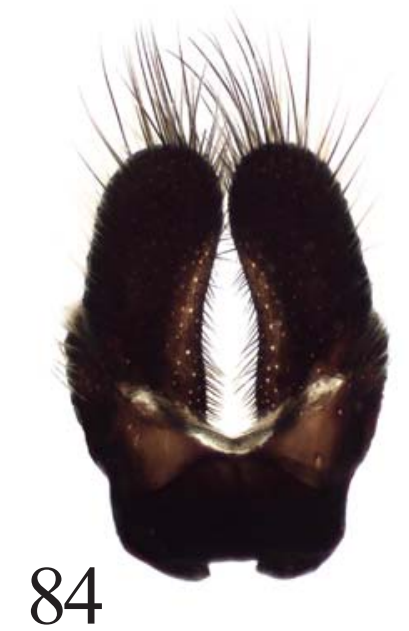

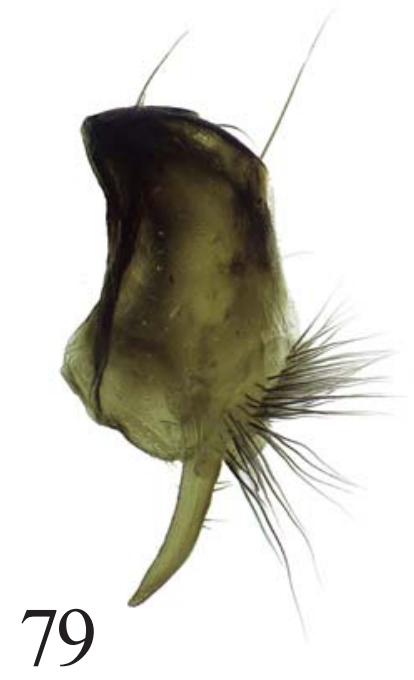
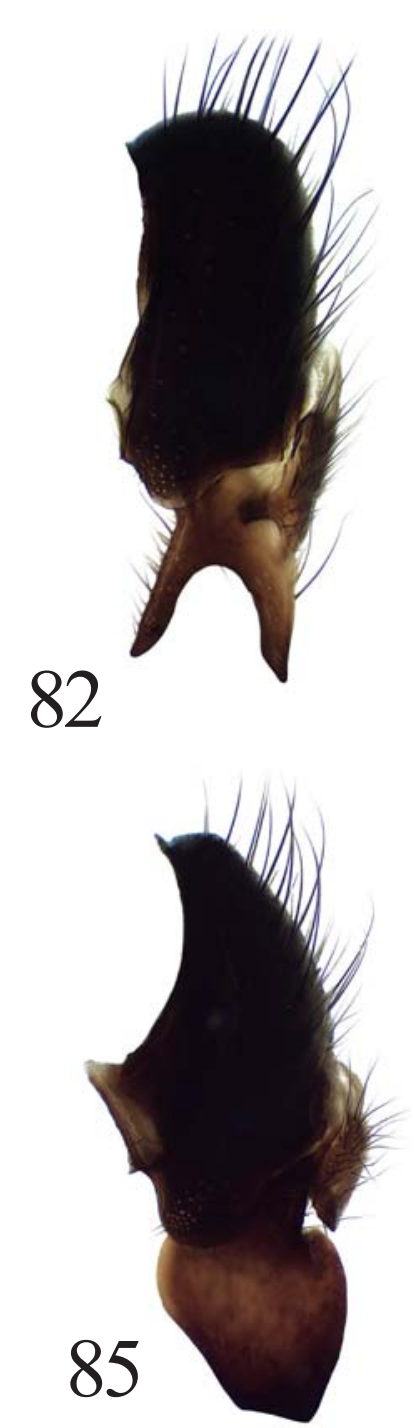
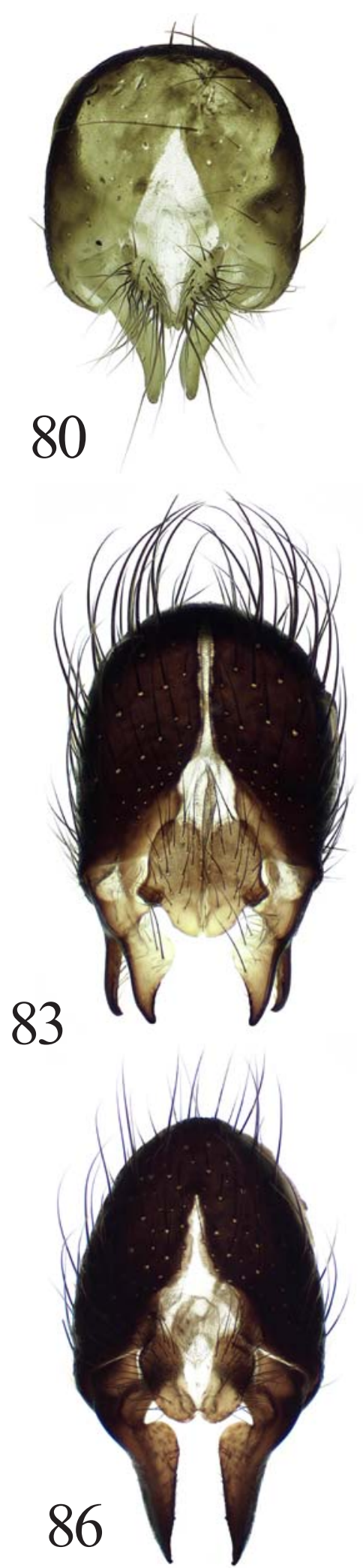

Figs 78-86. Cordilura nubilosa sp.n. (78-80), C picipes (Meigen) (81-83) and C. picticornis (Loew) (84-86): 78, 81, 84 - male sternite 5; 79, 82, 85 - epandrium and surstyli, lateral view; 80, 83, 86 - same, dorsal view.

Рис. 78-86. Cordilura nubilosa sp.n. (78-80), C. picipes (Meigen) (81-83) и C. picticornis (Loew) (84-86): 78, 81, 84- стернит 5 самца; 79, 82, 85 - эпандрий и сурстили, сбоку; 80, 83, 86 - то же, сверху. 
Vikhrev (1 9, ZMUM); Leningrad Oblast: Yashchera $\left(58.894^{\circ} \mathrm{N}\right.$ $29.820^{\circ} \mathrm{E}$ ), 23.VII.1957, A. Stackelberg (2 우, ZISP); Lake Lebyazh'e $\left(60.312^{\circ} \mathrm{N} 29.532^{\circ} \mathrm{E}\right), 29 . V .1899$, Bianki (1 ア , ZISP) same place, 20.V.1899, Bezval' (1 O, ZISP); Mordovia: Mordovsky Nature Reserve, River Pushta $\left(54.75^{\circ} \mathrm{N}, 43.20^{\circ} \mathrm{E}\right)$, 21.V.2020, N. Vikhrev (1 $\sigma^{7}$, ZMUM); Moscow and Moscow Oblast: Izmaylovo $\left(55.786^{\circ} \mathrm{N} 37.835^{\circ} \mathrm{E}\right), 15 . \mathrm{V} .1983$, A.L. Ozerov (1 $\sigma^{\top}$, ZMUM); Andreevskoe $\left(55.974^{\circ} \mathrm{N} 35.603^{\circ} \mathrm{E}\right), 27 . \mathrm{VI} .2007$, A.L. Ozerov (1 $\sigma^{7}, 2$, $2 \circ$, ZMUM); Golitsyno $\left(55.649^{\circ} \mathrm{N} 37.011^{\circ} \mathrm{E}\right)$, 27.VI.1978, A. Shatalkin (2 $\sigma^{\top} \sigma^{7}, 3$ 우, ZMUM); Dmitrov env. $\left(56.316^{\circ} \mathrm{N} 37.725^{\circ} \mathrm{E}\right), 19 . \mathrm{V} .2006,10-15 . \mathrm{V} ., 17$ and 28.VI.2007, 5 and 9.V.2008, N. Vikhrev (5 $\sigma^{7} \sigma^{7}, 3$ ox, ZMUM); Zosimova Pustyn' $\left(55.412^{\circ} \mathrm{N} 36.814^{\circ} \mathrm{E}\right), 17 . \mathrm{V} .2008$, D. Gavryushin (1 $\sigma^{7}$ ZMUM); Lugovaya $\left(56.203^{\circ} \mathrm{N} 37.823^{\circ} \mathrm{E}\right), 26$. VI.1993, A.L. Ozerov (1 9 , ZMUM); Naro-Fominsk $\left(55.357^{\circ} \mathrm{N} 36.736^{\circ} \mathrm{E}\right), 13$ and 16.V.2006, D. Gavryushin (3 $\sigma^{7} \sigma^{7}, 1$, ZMUM); Ozhigovo $\left(55.453^{\circ} \mathrm{N} 36.884^{\circ} \mathrm{E}\right), 22$.VII.2007, 10.V.2008, 10.VIII.2009, D Gavryushin (1 $\sigma^{\top}, 3$ 웅. ZMUM); Smolevo $\left(55.578^{\circ} \mathrm{N} 38.666^{\circ} \mathrm{E}\right)$, 6-7.VI.2011, K. Tomkovich (1 $\sigma^{7}$, ZMUM); Stepan'kovo $\left(55.004^{\circ} \mathrm{N}\right.$ $\left.35.624^{\circ} \mathrm{E}\right), 8$. VII.2006, 3.VI.2007, A.L. Ozerov (5 $0^{7} 0^{7}, 1$, ZMUM); Yur'evo (56.006 $\left.{ }^{\circ} \mathrm{N} 35.546^{\circ} \mathrm{E}\right)$, 9.VIII.2007, 29.VII.2009, A.L. Ozerov ( $\left.2 \bigcirc^{\top} \sigma^{7}, Z M U M\right)$; Yakshino env. $\left(55.920^{\circ} \mathrm{N} 35.573^{\circ} \mathrm{E}\right)$, 15.V.2015, A.L. Ozerov (1 $\Im^{7}, 1$ ․ ZMUM); Snegiri $\left(55.887^{\circ} \mathrm{N}\right.$ $\left.37.028^{\circ} \mathrm{E}\right), 26 . V .1984$, Gorodkov ( $2 \sigma^{\top} \sigma^{\top}, 2$ o 2 , ZISP); Novgorod Oblast: Ramen'e $\left(58.374^{\circ} \mathrm{N} 33.430^{\circ} \mathrm{E}\right)$, Okulovka env., 4.VI.1988, Gorodkov (2 $\sigma^{7} \mathrm{O}^{7}, 1$, , ZISP); Vorohezh Oblast: Usmansky Bor $\left(51.911^{\circ} \mathrm{N} 39.562^{\circ} \mathrm{E}\right), 15$. VII.1968, Negrobov (1 9 , ZISP); Yaroslavl' Oblast: Berditsino $\left(57.455^{\circ} \mathrm{N} 40.107^{\circ} \mathrm{E}\right), 13 . \mathrm{VI} .1906,20 . \mathrm{V}$. and 29.VI.1907, A. Yakovlev ((3 O $\sigma^{7}$, ZISP).

DIAGNOSTIC DESCRIPTION. Male. Female (Fig. 2). Body-length 4.5-6.4 mm. Head. Frontal vitta dark brown or black, with whitish reflection; fronto-orbital plate black, greyish dusted. Face and parafacial yellowish with whitish reflection. Gena blackish. Postcranium black, grey dusted. 3 orbital and 2 frontal setae present. Antenna black. Postpedicel approximately 2 times as long as wide. Arista plumose, the longest hairs approximately equal to the width of postpedicel. Palpus black. Thorax black, delicately grey dusted. Acrostichals setulose in two rows, dorsocentrals $3+3$ (including small scapular seta), intra-alars $(0-1)+(1-2)$, supra-alars $1+2,2$ postpronotals. Scutellum with a pair of strong discal setae only; apical scutellar setae as setulae or absent. Legs greyish dusted. Fore coxa black. Femora and tibiae of all legs black. Tarsi of all legs brownish or black. Wing tinged with brownish; veins blackish. Vein $\mathrm{R}_{1}$ setulose on apical third of dorsal surface. Abdomen black, delicately grey dusted. Male sternite 4 rectangular, wider than long. Male sternite 5, epandrium and surstyli as in Figs 81-83.

DISTRIBUTION. Russia: Altay, Karelia, Kursk Oblast, Leningrad Oblast, Mordovia, Moscow Oblast, Novgorod Oblast, Vorohezh Oblast, Yaroslavl' Oblast. - Europe, Mongolia [Gorodkov, 1986: 14].

\section{Cordilura picticornis (Loew, 1864)} Figs 84-86.

picticornis Loew, 1864: 22 (Cordylura). Type-locality: "Sibiria" (Russia).

latigenis Hendel, 1930: 3 (Cordylura). Type-locality: "Petropavlovsk" (Russia, Kamchatka Krai).

REMARKS. The species was described by Loew [1864: 22] from Siberia without indicating a specific locality, after registered for Russia by Hendel [1930: 3, as
C. latigenis] from Kamchatka Krai. Noted by Gorodkov [1970: 448] from Kola Peninsula and Polar Ural, also from Siberia and Far East, but without indicating specific locality [Gorodkov, 1986: 15]. Registered from Chukotka and Magadan Oblast [Ozerov, Krivosheina, 2014: 211] and from Yakutia [Bagachanova et al., 2016: 779].

MATERIAL EXAMINED. Altai: Kosh-Agach, plato Ukok, $2450 \mathrm{~m}$, Lake Muzdy-Bulak (49.3ㅇ $\left.87.65^{\circ} \mathrm{E}\right)$, 8.VII.2008, A. Barkalov ( $9 \bigcirc^{7} \sigma^{7}, 2$ \% , ISEA and ZMUM); the source of the River Naryn-Gol from Lake Kindyktykul', 2477 m, $\left(49.83^{\circ} \mathrm{N}\right.$, $\left.89.44^{\circ} \mathrm{E}\right), 19$. VII.2009, A. Barkalov ( $3 \sigma^{7} \sigma^{7}, 1$ ㅇ, ISEA); Kuray env. $\left(50.233^{\circ} \mathrm{N} 87.930^{\circ} \mathrm{E}\right), 22-24$.VII.2006, A. Ovchinnikov (1 $\sigma^{7}$, ZISP); Kosh-Agach $\left(49.997^{\circ} \mathrm{N} 88.670^{\circ} \mathrm{E}\right), 24 . \mathrm{VI}$. and 31.VII.1964, Nartshuk (2 $\sigma^{7}$, ZISP); Arkhangelsk Oblast: Nar'yan-Mar $\left(67.627^{\circ} \mathrm{N}, 53.000^{\circ} \mathrm{E}\right)$, 7.VII.2008, A.L. Ozerov (2 $\sigma^{7} \sigma^{\top}, 4$ 우, ZMUM); Nar'yan-Mar $\left(67.636^{\circ} \mathrm{N} 53.031^{\circ} \mathrm{E}\right), 2$ and

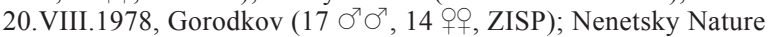
Reserve, cordon "Bol'shoy Gusinets" $\left(68.175^{\circ} \mathrm{N}, 53.645^{\circ} \mathrm{E}\right)$, 10.VII.2008, A.L. Ozerov, N. Vikhrev (3 $\sigma^{7} \sigma^{\top}$, ZMUM); the lower reaches of the River Pechora, Kashin I. $\left(68.242^{\circ} \mathrm{N}\right.$, $\left.53.856^{\circ} \mathrm{E}\right), 10 . \mathrm{VII} .2008$, A.L. Ozerov ( $3 \sigma^{7} \sigma^{\top}, 1$ ㅇ, ZMUM); the lower reaches of the River Pechora $\left(68.334^{\circ} \mathrm{N} 53.304^{\circ} \mathrm{E}\right)$, 11.VII.2008, A.L. Ozerov (1 9 , ZMUM); Karpogory (ca. $64^{\circ} \mathrm{N}$ $44.45^{\circ} \mathrm{E}$ ), River Pinega, 10.VII.1996, Gorodkov (2 우, ZISP); Nizhnyaya Pesha $\left(66.751^{\circ} \mathrm{N} 47.760^{\circ} \mathrm{E}\right), 23$.VII., 2 and 22.VIII.1978, Gorodkov ( $\sigma^{7} \sigma^{\top}, 4$ 우, ZISP); Chukotka: bank of the River Anadyr $\left(64.72^{\circ} \mathrm{N} 175.21^{\circ} \mathrm{E}\right), 25$. VI.-19.VII.2014, A. Barkalov (2 90 , ZMUM); Kamchatka Krai: Uzon $\left(54.503^{\circ} \mathrm{N}\right.$ $\left.159.99^{\circ} \mathrm{E}, 54.487^{\circ} \mathrm{N} 159.977^{\circ} \mathrm{E}\right), 16$ and 17. VII., 1.VIII.1977, Stenchenko (1 Or, 2 우, ZMUM), same place, 11.VII.1987, 20.VII.2009, L. Lobkova (2 $\sigma^{\top} \sigma^{\top}, 2$ ơ, ZMUM); Valley of Geysers (ca. $\left.54.43^{\circ} \mathrm{N} 160.15^{\circ} \mathrm{E}\right), 12$.VIII.1985, V. Zlobin (1 $\sigma^{7}, 1$, ZISP); Lake Nerpich'e (ca. $\left.56.326^{\circ} \mathrm{N} 162.684^{\circ} \mathrm{E}\right)$, Khalmychensky Cape, 10.VII.1909, Derzhavin (3 $\sigma^{7} \sigma^{7}$, ZISP); Ust'-Kamchatsk $\left(56.229^{\circ} \mathrm{N} 162.469^{\circ} \mathrm{E}\right), 15$.VII.1909, A. Derzhavin $\left(1 \sigma^{7}\right.$, ZISP); Korf $\left(60.376^{\circ} \mathrm{N} 166.023^{\circ} \mathrm{E}\right), 29 . \mathrm{VI} .1959$, Gorodkov (1 フ7', ZISP); Lake Kultuchnoe $\left(53.03^{\circ} \mathrm{N}, 158.64^{\circ} \mathrm{E}\right), 5-9$ VII.2020, F. Martynovchenko (1 $\sigma^{7}$, ZMUM); Karelia: Primorskiy $\left(66.546^{\circ} \mathrm{N}\right.$ 33.103 ${ }^{\circ}$ E), 4-6.VII.2010, A.L. Ozerov (1 $\sigma^{7}, 6$ oO, ZMUM); Khabarovsk Krai: Shantarskie Islands (ca. $54.922^{\circ} \mathrm{N} 137.683^{\circ} \mathrm{E}$ ), 28.VII.1911, V.K. Soldatov (1 $\sigma^{7}$, ZISP); Komi: Shchel'yabozh

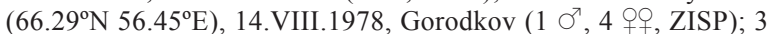
$\mathrm{km} \mathrm{N}$ of Ust-Tsilma (ca. $65.45^{\circ} \mathrm{N} 52.11^{\circ} \mathrm{E}$ ), Roshchinskiy, 10.VIII.1978, Gorodkov (2 90 , ZISP); Ust-Tsilma $\left(65.440^{\circ} \mathrm{N}\right.$ $\left.52.153^{\circ} \mathrm{E}\right)$, 9.VIII.1978, Gorodkov (1 9 , ZISP); Krasnoyarsk Krai: Igarka $\left(67.457^{\circ} \mathrm{N} 86.598^{\circ} \mathrm{E}\right)$, River Yenisei, 30.VI.1967, Gorodkov (1 + , ZISP); Magadan Oblast: $\left(59.568^{\circ} \mathrm{N} 150.809^{\circ} \mathrm{E}\right)$, 27.VII.1963, Zhelokhovtsev (1 $\circ$, ZMUM); $30 \mathrm{~km} \mathrm{~W}$ of Magadan Town $\left(59.683^{\circ} \mathrm{N} 150.333^{\circ} \mathrm{E}\right), 28$.VI.2017, V. Sorokina ( 1 ㅇ ISEA); Koni peninsula, Plosky Cape $\left(59.161^{\circ} \mathrm{N} 151.643^{\circ} \mathrm{E}\right)$, 3.VII.2016 and 7.VII.2017, N. Tridrich (2 $\sigma^{7} \sigma^{7}$, ZMUM); Sakhalin Oblast: Paramushir I., Severo-Kuril'sk $\left(50.679^{\circ} \mathrm{N} 156.132^{\circ} \mathrm{E}\right)$, 5.IX.1968, Gorodkov (1 $\sigma^{7}$, ZISP); Sakhalin I., Nadezhda Bay (ca. $49.273^{\circ} \mathrm{N}$ 142.074 ${ }^{\circ}$ ), 9.VII.1908, Soldatov (1 $\sigma^{7}$, ZISP); Tyumen' Oblast: Shapsha env. $\left(61.085^{\circ} \mathrm{N} 69.458^{\circ} \mathrm{E}\right), 14-16$. VII.2010, K. Tomkovich $\left(1\right.$ ㅇ, ZMUM); Gaz-sale $\left(67.364^{\circ} \mathrm{N} 78.998^{\circ} \mathrm{E}\right), 18 \mathrm{~km}$ SE of Tazovskoe, 31.VII.1977, Gorodkov (1 $\sigma^{7}, 1$ ㅇ, ZISP); Yakutia: the mouth of the River Vilyuy $\left(64.285^{\circ} \mathrm{N} 126.386^{\circ} \mathrm{E}\right), 8-$ 16.VI.1875, Chekanovskiy ( $3 \sigma^{\top} \sigma^{7}, 1$ ㅇ, ZISP); Cherskiy $\left(68.750^{\circ} \mathrm{N}\right.$ 161.332 ${ }^{\circ}$ E), 4.VII.1971, Chelnokov (13 $\sigma^{7} \sigma^{7}, 3$ oᄋ, ZISP).

DIAGNOSTIC DESCRIPTION. Male. Female. Body-length 6.2-9.4 mm. Head. Frontal vitta yellow or reddish yellow, with whitish reflection; fronto-orbital plate black, greyish dusted. Face and parafacial yellow with golden reflection. Gena from yellow to brown. Postcranium black, grey dusted. 3 orbital and 4-5 frontal setae present. Antenna black. Scapus and pedicel yellowish or brownish; postpedicel usually yellowish basally and black in apical part, approximately 1.5-2 


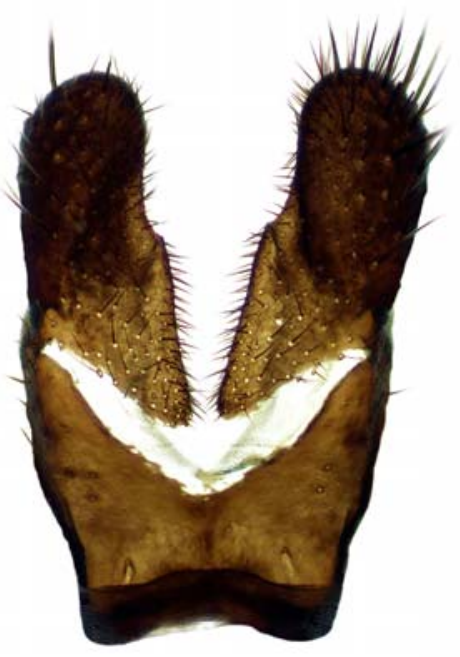

87

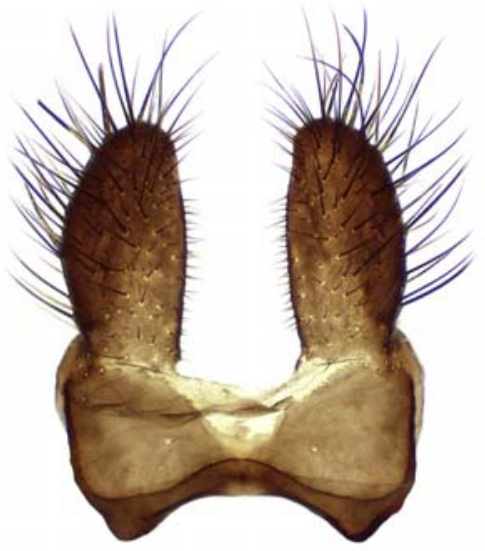

90

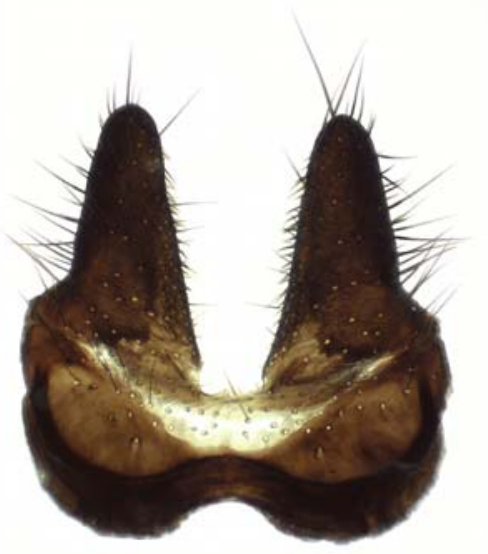

93
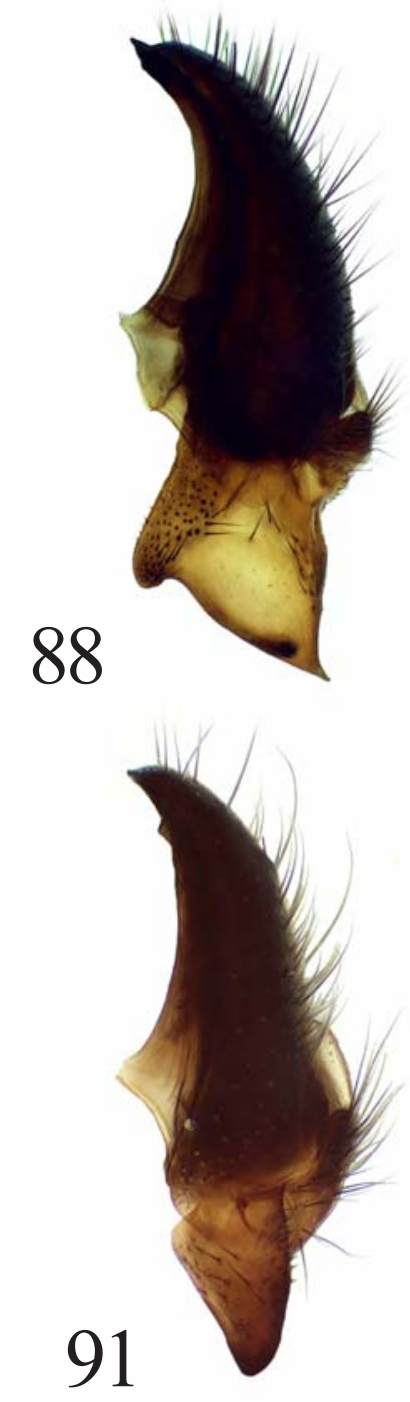

89
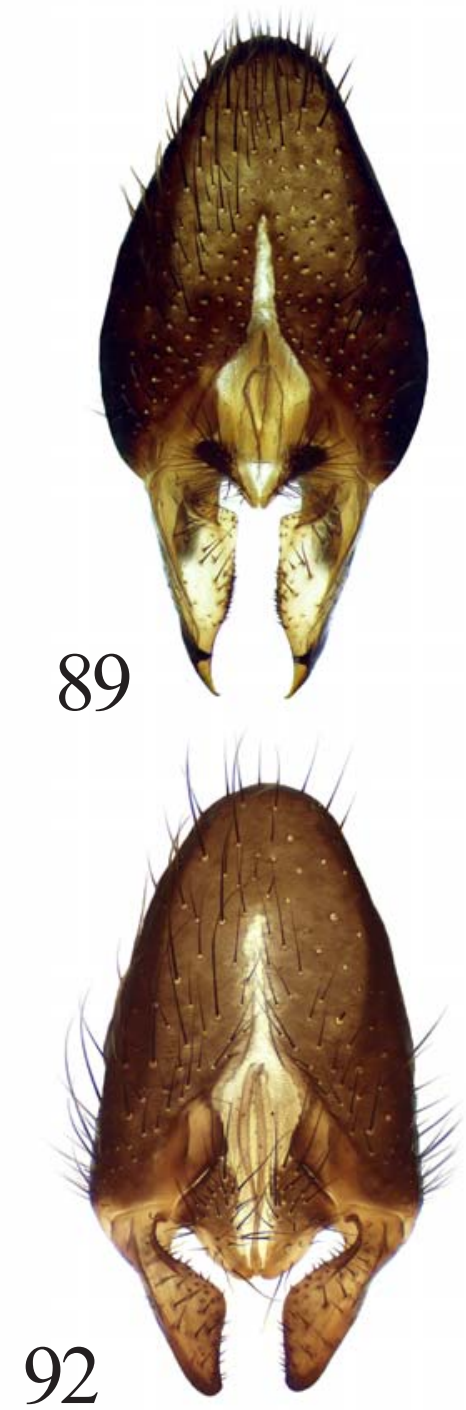

92
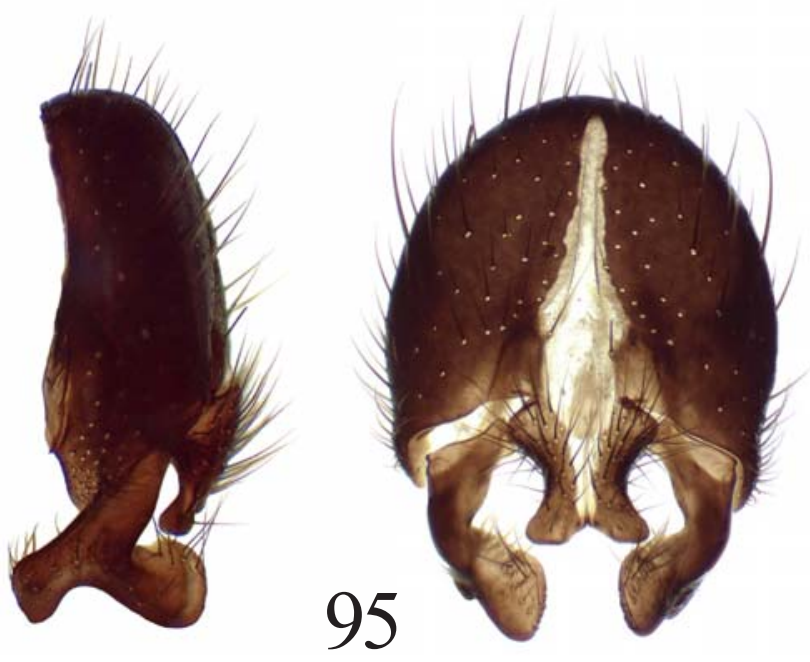

Figs 87-95. Cordilura proboscidea (Zetterstedt) (87-89), C. pudica (Meigen) (90-92) and C. remmi Elberg (93-95): 87, 90, 93 male sternite 5; 88, 91, 94 - epandrium and surstyli, lateral view; 89, 92,95 - same, dorsal view. 93-95 — after Ozerov \& Krivosheina, 2017, figs 10-12.

Рис. 87-95. Cordilura proboscidea (Zetterstedt) (87-89), С. pudica (Meigen) (90-92) и С. remmi Elberg (93-95): 87, 90, 93 - стернит 5 самца; 88, 91, 94 - эпандрий и сурстили, сбоку; 89, 92, 95 — то же, сверху. 93-95 — по Ozerov \& Krivosheina, 2017 , figs $10-12$. 
times as long as wide. Arista plumose, the longest hairs approximately equal to the width of postpedicel. Palpus yellow. Thorax black, grey dusted. Acrostichals setulose in two rows, dorsocentrals $3+3$ (including small scapular seta), intra-alars $1+2$, supra-alars $1+2,2$ postpronotals. Scutellum with a pair of strong discal and a pair of strong apical setae. Legs greyish dusted. Fore femur blackish basally and yellow apically or black completely; mid and hind femora from yellow completely to black completely. Tibiae of all legs yellow. Tarsi of all legs from yellow to blackish. Wing tinged with yellow; veins brownish. Vein $\mathrm{R}_{1}$ setulose on apical third of dorsal surface. Abdomen black, grey dusted. Male sternite 4 trapezoid, slightly wider than long. Male sternite 5, epandrium and surstyli as in Figs 84-86.

DISTRIBUTION. Russia: Altai, Arkhangelsk Oblast, Chukotka, Kamchatka Krai, Karelia, Khabarovsk Krai, Komi, Krasnoyarsk Kray, Magadan Oblast, Sakhalin Oblast, Tyumen' Oblast, Yakutia. - Europe, North America.

\section{Cordilura proboscidea (Zetterstedt, 1838)} Figs 16, 87-89.

proboscidea Zetterstedt, 1838: 728 (Cordylura). Type-locality: "Tromsoe ... Nordlandiae ... Wittangi Lapponiae Tornensis" (Norway, Sweden).

REMARK. Noted by Gorodkov [1970: 446, 1986: 15] for north of European part of Russia (Kola Peninsula, Polar Ural) and for north of Siberia, but without indicating specific locality.

MATERIAL EXAMINED. Komi: Sivaya Maska station $\left(66.675^{\circ} \mathrm{N} 62.569^{\circ} \mathrm{E}\right), 5 \mathrm{~km} \mathrm{NW}, 14 . \mathrm{VII} .1961$, Gorodkov $\left(2 \mathrm{O}^{7} \mathrm{O}^{7}, 4\right.$ 우, ZISP); Krasnoyarsk Krai: Igarka $\left(67.457^{\circ} \mathrm{N} 86.598^{\circ} \mathrm{E}\right)$, River Yenisei, 1-3.VII.1967, Gorodkov (3 $0^{7} \sigma^{7}, 6$ 오, ZISP); Tyumen' Oblast: Obdorsk [now $=$ Salekhard] $\left(66.53^{\circ} \mathrm{N} 66.6135^{\circ} \mathrm{E}\right)$ 25.VI.1914, Shukhov ( $1 \mathrm{O}^{7}$, ZISP); Tobol'sk $\left(58.206^{\circ} \mathrm{N} 68.264^{\circ} \mathrm{E}\right)$, 22.V.1925, Fridolin $\left(2 \mathrm{O}^{7} \mathrm{O}^{7}\right.$, ZISP); Yakutia: Kyusyur $\left(70.687^{\circ} \mathrm{N}\right.$ 127.366 E), 14.VII.1957, Gorodkov (1 $0^{7}, 1$ ㅇ, ZISP).

DIAGNOSTIC DESCRIPTION. Male. Female. Body-length 6.8-7.8 mm. Head. Frontal vitta yellow; fronto-orbital plate black, greyish dusted. Face, parafacial and gena yellowish with whitish reflection. Postcranium black, greyish dusted. 3 orbital and 2-4 frontal setae present. Antenna blackish; postpedicel approximately $1.5-2$ times as long as wide. Arista nearly bare, longest hairs not exceeding greatest diameter of arista. Palpus black. Thorax black, greyish dusted. Acrostichals setulose in two rows, dorsocentrals (4$5)+3$ (including scapular seta), intra-alars $1+2$, supraalars $1+2,2$ postpronotals. Scutellum with a pair of strong discal and a pair of strong apical setae. Legs greyish dusted. Femora of all legs black; tibiae of all legs from yellow to blackish; tarsi of all legs yellow. Wing tinged with light brownish; veins brownish. Vein $\mathrm{R}_{1}$ bare on apical third of dorsal surface. Abdomen black, greyish dusted. Male sternite 4 rectangular, wider than long (Fig. 16). Male sternite 5, epandrium and surstyli as in Figs 87-89.

DISTRIBUTION. Russia: Komi, Krasnoyarsk Krai, Tyumen’ Oblast, Yakutia. — Europe, North America.

\section{Cordilura pudica (Meigen, 1826)}

Figs 13, 90-92.

pudica Meigen, 1826: 231 (Cordylura). Type-locality: not given. geniculata Zetterstedt, 1846: 1997 (Cordylura). Type-localities: "Scania ... Smolandia ... Helsingia ... Lapponia Umensi ..." (Sweden)

REMARKS. This species was recorded in Russia from Yaroslavl' Oblast [Ovchinnikov, 2004: 422], from Amur Oblast, Primorsky Krai and Sakhalin Oblast [Ozerov, Krivosheina, 2014: 211], from Yakutia [Bagachanova et al., 2016: 779]; noted by Gorodkov [1970: 446, 1986: 15] for European part of Russia without indicating specific locality.

MATERIAL EXAMINED. Altai: Artybash env., floodplain of the River Sema, $550 \mathrm{~m},\left(51.87^{\circ} \mathrm{N}, 87.18^{\circ} \mathrm{E}\right), 22$. VI.2009, V. Sorokina (1 9 , ISEA); Arkhangelsk Oblast: Nar'yan-Mar $\left(67.631^{\circ} \mathrm{N}\right.$ $\left.52.985^{\circ} \mathrm{E}, 67.627^{\circ} \mathrm{N} 53.000^{\circ} \mathrm{E}\right), 7-8 . \mathrm{VII} .2008$, A.L. Ozerov (2 $0^{7} 0^{7}$, 3 우 $\mathrm{ZMUM}$ ); Nenetsky Nature Reserve, cordon "Bol'shoy Gusinets" $\left(68.175^{\circ} \mathrm{N}, 53.645^{\circ} \mathrm{E}\right), 9-10 . \mathrm{VII} .2008$, A.L. Ozerov (2 $\mathrm{O}^{7} \sigma^{7}$, 1 \%, ZMUM); the lower reaches of the River Pechora $\left(68.334^{\circ} \mathrm{N}\right.$, 53.304 ${ }^{\circ}$ E), 11.VII.2008, A.L. Ozerov (1 $\sigma^{7}$, ZMUM); Solvychegodsk $\left(61.342^{\circ} \mathrm{N} 46.913^{\circ} \mathrm{E}\right), 12-13$. VIII.2010, D. Gavryushin (3 $\mathrm{O}^{7} \mathrm{O}^{7}, 3$ 우, ZMUM); Arkhangelsk $\left(64.546^{\circ} \mathrm{N} 40.567^{\circ} \mathrm{E}\right)$, 5.VIII.2010, D. Gavryushin (1 + , ZMUM); $73 \mathrm{~km} \mathrm{NW}$ of Nar'yanMar (ca. $67.981^{\circ} \mathrm{N} 51.608^{\circ} \mathrm{E}$ ), 3.VIII.1978, Gorodkov (2 우, ZISP); Nizhnyaya Pesha $\left(66.751^{\circ} \mathrm{N} 47.760^{\circ} \mathrm{E}\right), 22$.VIII.1978, Gorodkov (3 $\mathrm{O}^{7} \mathrm{O}^{7}, 1$ \%, ZISP); Solovetskie Islands $\left(65.080^{\circ} \mathrm{N} 35.686^{\circ} \mathrm{E}\right)$, 10.VI.1895, Birulya (1 ㅇ, ZISP); Karpogory (ca. $64^{\circ} \mathrm{N} 44.45^{\circ} \mathrm{E}$ ), River Pinega, 6.VII.1996, Gorodkov ( $10^{7}, 1$ \%, ZISP); Solovetskiy I. $\left(65.080^{\circ} \mathrm{N} 35.686^{\circ} \mathrm{E}\right), 27$.VII.1959, Gorodkov (1 ㅇ, ZISP); Bashkiria: E of Kryktytau $\left(53.525^{\circ} \mathrm{N} 58.498^{\circ} \mathrm{E}\right), 2-8$.VIII.2008, K. Tomkovich ( $1 \sigma^{7}, 1$, ZMUM); Buryatia: Khargun, $6 \mathrm{~km}$ E of Kyren $\left(51.683^{\circ} \mathrm{N} 102.132^{\circ} \mathrm{E}\right)$, Sayany, 11.VII.1965, Gorodkov (1 9 , ZISP); Chukotka: bank of the River Anadyr $\left(64.72^{\circ} \mathrm{N} 175.21^{\circ} \mathrm{E}\right), 25$.VI.19.VII.2014, A. Barkalov (1 $\sigma^{7}$, ISEA); Karelia: Primorskiy $\left(66.546^{\circ} \mathrm{N} 33.103^{\circ} \mathrm{E}\right), 5 . \mathrm{VII} .2010$, A.L. Ozerov $\left(6 \sigma^{7} \sigma^{7}, 3\right.$ OO, ZMUM); Poyakonda $\left(66.589^{\circ} \mathrm{N} 33.821^{\circ} \mathrm{E}\right), 8$.VII.2010, A.L. Ozerov $\left(1 \sigma^{7}, Z_{M U M}\right)$; Kaskesnavolok $\left(61.594^{\circ} \mathrm{N} 33.323^{\circ} \mathrm{E}\right)$, 20.VI.1979, Gorodkov (8 $0^{7} \sigma^{7}, 2$ 우, ZISP); Kizhi $\left(62.084^{\circ} \mathrm{N}\right.$ $\left.35.211^{\circ} \mathrm{E}\right), 19$.VI.1979, Gorodkov (6 $\mathrm{O}^{7} \mathrm{O}^{7}, 2$ 2 ,, ZISP); Khabarovsk Krai: Lake Sindinskoe $\left(48.92^{\circ} \mathrm{N} 136.24^{\circ} \mathrm{E}\right), 7 . V I .2014, \mathrm{~N}$. Vikhrev $\left(1\right.$ 9, ZMUM); River Khicha $\left(49.05^{\circ} \mathrm{N} 139.43^{\circ} \mathrm{E}\right), 10 . \mathrm{VI} .2014, \mathrm{~N}$. Vikhrev (1 9 , ZMUM); Komi: Ust-Tsilma $\left(65.440^{\circ} \mathrm{N} 52.153^{\circ} \mathrm{E}\right)$, 11.VIII.1978, Gorodkov (1 9 , ZISP); Inta $\left(66.04^{\circ} \mathrm{N} 60.17^{\circ} \mathrm{E}\right)$, 5.VIII.1985, Gorodkov (4 우, ZISP); Ust'-Ukhta $\left(63.620^{\circ} \mathrm{N}\right.$ $\left.53.878^{\circ} \mathrm{E}\right)$, 4.VIII.1985, Gorodkov (1 $\mathrm{O}^{7}$, ZISP); Blagoevo $\left(63.424^{\circ} \mathrm{N}\right.$, 47.952 ${ }^{\circ}$ E), 12.VII.1996, Gorodkov (3 우, ZISP); Sivaya Maska station $\left(66.675^{\circ} \mathrm{N} 62.569^{\circ} \mathrm{E}\right), 5 \mathrm{~km} \mathrm{NW}, 17$. VII.1961, Gorodkov $(2$ $\mathrm{O}^{7} \sigma^{7}, 5$ 우, ZISP); Krasnoyarsk Krai: Igarka $\left(67.457^{\circ} \mathrm{N} 86.598^{\circ} \mathrm{E}\right)$, River Yenisei, 1.VII.1967, Gorodkov ( $2 \sigma^{7} \sigma^{7}, 9$ 우, ZISP); Leningrad Oblast: Gatshina $\left(59.56^{\circ} \mathrm{N} 30.13^{\circ} \mathrm{E}\right), 10$.VI.1940, A. Stackelberg (1 $0^{7}$, ZISP); Luga (ca. $58.73^{\circ} \mathrm{N} 29.84^{\circ} \mathrm{E}$ ), 9.VIII.1954, A. Stackelberg (4 $O^{7} \sigma^{7}$, ZISP); Moscow and Moscow Oblast: Izmaylovo $\left(55.802^{\circ} \mathrm{N} 37.844^{\circ} \mathrm{E}\right)$, 20.VI.2007, A.L. Ozerov (2 우, ZMUM); Kosino $\left(55.721^{\circ} \mathrm{N} 37.847^{\circ} \mathrm{E}\right)$, 26.VI, 24-31.VII., 2.VIII, 8.IX.1921, B. Dodonov (2 $0^{7} 0^{7}, 6$ 우, ZMUM); Skhodnya $\left(55.842^{\circ} \mathrm{N} 37.412^{\circ} \mathrm{E}\right)$, 3.VII.1936, B. Rohdendorf (1 9 , ZMUM); $20 \mathrm{~km}$ WSW of Volokolamsk $\left(55.983^{\circ} \mathrm{N} 35.616^{\circ} \mathrm{E}\right), 11 . \mathrm{VII} .1999$, A.L. Ozerov $(2$ 웅, ZMUM); Abramtsevo $\left(56.23^{\circ} \mathrm{N} 37.956^{\circ} \mathrm{E}\right), 13$ and 26.VIII.1955, 22.VII.1957, E.S. Smirnov (2 $0^{7} 0^{9}, 1$ \% ZMUM); Andreevskoe env. $\left(55.974^{\circ} \mathrm{N} 35.604^{\circ} \mathrm{E}, 55.985^{\circ} \mathrm{N} 35.620^{\circ} \mathrm{E}, 55.985^{\circ} \mathrm{N} 35.622^{\circ} \mathrm{E}\right.$, $\left.55.978^{\circ} \mathrm{N} 35.588^{\circ} \mathrm{E}\right), 15-16$. VII.2006, 5.VI. and 9.VIII.2007, A.L. Ozerov (11 $0^{7} 0^{7}, 4$ 우, ZMUM); Burtsevo env. (55.976 ${ }^{\circ} \mathrm{N} 35.590^{\circ} \mathrm{E}$, $\left.55.978^{\circ} \mathrm{N} 35.590^{\circ} \mathrm{E}\right), 14 . \mathrm{VIII} .2008$, 26.VII.2009, A.L. Ozerov (4 $\mathrm{O}^{7} \mathrm{O}^{7}, 2$ 우, ZMUM); Dmitrov env. (56.316 $\left.\mathrm{N} 37.725^{\circ} \mathrm{E}\right), 10$ and 15.V., 9.VII.2007, N. Vikhrev (3 O $^{7} 0^{7}, 1$ ․, ZMUM); Mamontovka $\left(55.999^{\circ} \mathrm{N} 37.816^{\circ} \mathrm{E}\right), 30 . \mathrm{VII} .1956$, E. Smirnov (1 +, ZMUM); Molzhaninovka $\left(55.936^{\circ} \mathrm{N} 37.385^{\circ} \mathrm{E}\right), 13 . V .2010$, A.L. Ozerov (1 +, ZMUM); Naro-Fominsk $\left(55.357^{\circ} \mathrm{N} 36.736^{\circ} \mathrm{E}\right), 13 . V .2006$, D. 
Gavryushin (1 $\sigma^{7}$, ZMUM); Pavlovskoe env. (55.921 $\left.{ }^{\circ} \mathrm{N} 35.588^{\circ} \mathrm{E}\right)$, 14.VIII.2006, A.L. Ozerov (1 ऽ', ZMUM); Snegiri $\left(55.887^{\circ} \mathrm{N}\right.$ $\left.37.028^{\circ} \mathrm{E}\right), 26 . V .1984$, Gorodkov ( $2 \mathrm{\sigma}^{\top} \sigma^{7}, 1$ \%, ZISP); Yur'evo env. $\left(56.006^{\circ} \mathrm{N} 35.546^{\circ} \mathrm{E}, 55.005^{\circ} \mathrm{N} 35.545^{\circ} \mathrm{E}\right), 9$. VIII.2007, 29.VII.2009, A.L. Ozerov ( $1 \sigma^{\top}, 2$, $\circ$, ZMUM); Murmansk Oblast: Murmansk env. $\left(68.976^{\circ} \mathrm{N} 33.119^{\circ} \mathrm{E}\right), 18-21$.VII.2011, A. Ozerov $\left(1 \sigma^{\top}, 2\right.$, 2 , ZMUM); Novgorod Oblast: Ramen'e $\left(58.374^{\circ} \mathrm{N} 33.430^{\circ} \mathrm{E}\right)$, Okulovka env., 4.VI.1988, Gorodkov (1 o, ZISP); Novosibirsk: $\left(54.825^{\circ} \mathrm{N} 83.114^{\circ} \mathrm{E}\right), 18 . V I .2009$, O. Kosterin (1 $\sigma^{7}$, ZMUM); Novosibirsk Oblast: Zherebtsovo env. $\left(55.125^{\circ} \mathrm{N} 83.256^{\circ} \mathrm{E}\right)$, 22.VI.2006, O. Kosterin (1 + , ZMUM); Primorsky Krai: Kamenushka $\left(43.62^{\circ} \mathrm{N} 132.23^{\circ} \mathrm{E}\right), 22-24 . V I .2014$, N. Vikhrev (1 웅 ZMUM); Sakhalin Oblast: Kunashir I., Mendeleev Volcano env. $\left(43.961^{\circ} \mathrm{N} 145.729^{\circ} \mathrm{E}\right), 21 . \mathrm{VI} .1985$, S. Churkin (1 q, ZMUM); Kunashir I., Kurilsky Nature Reserve, cordon Andreevskyi (45.54 ${ }^{\circ} \mathrm{N}$ $\left.145.37^{\circ} \mathrm{E}\right)$, 8.VII.2014, T.V. Galinskaya (2 90, ZMUM); Saratov Oblast: Nizhnyaya Bannovka $\left(50.666^{\circ} \mathrm{N}, 45.633^{\circ} \mathrm{E}\right), 1-2$.VII.2003, V. Krivokhatskiy, O. Ovchinnikova (1 +, ZISP); Sverdlov Oblast: Ekaterinburg, Uktus station $\left(56.780^{\circ} \mathrm{N} 60.628^{\circ} \mathrm{E}\right)$, 19.VI.1910, Yakobson (1 9 , ZISP); Tver' Oblast: Ostashkov $\left(57.140^{\circ} \mathrm{N} 33.163^{\circ} \mathrm{E}\right), 13 . \mathrm{VI} ., 14$. VII.1936, B. Rohdendorf $\left(2 \sigma^{7} \sigma^{7}\right.$ 1 , ZMUM); Tyumen' Oblast: Shapsha env. $\left(61.085^{\circ} \mathrm{N}\right.$ 69.458 E), 14-16.VII.2010, K. Tomkovich (1 $\sigma^{7}$, ZMUM); Saran-

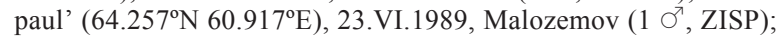
Yakutia: Verkhoyansk $\left(67.548^{\circ} \mathrm{N} 133.396^{\circ} \mathrm{E}\right)$, 23.VII.1974, Gorodkov ( 1 , ZISP) Yaroslavl' Oblast: Berditsyno $\left(57.455^{\circ} \mathrm{N}\right.$ $\left.40.107^{\circ} \mathrm{E}\right), 17$. VI. and 8.VII.1906, 3.VII.1907, A. Yakovlev (7 $\sigma^{7} \sigma^{7}, 6$ OO, ZISP).

ADDITIONAL MATERIAL EXAMINED. Belarus: Belovezhskaya Pushcha $\left(52.707^{\circ} \mathrm{N} 23.862^{\circ} \mathrm{E}\right), 17$. VII.1961, Nartshuk (1 $\sigma^{7}$, ZISP)

DIAGNOSTIC DESCRIPTION. Male. Female. Bodylength $6.2-8.6 \mathrm{~mm}$. Head. Frontal vitta from reddish yellow to blackish, with whitish reflection; fronto-orbital plate black, greyish dusted. Face and parafacial yellow with whitish reflection. Gena from yellow to blackish. Postcranium black, greyish dusted. 3 orbital and 2-3 frontal setae present. Antenna black. Postpedicel approximately 2-2.5 times as long as wide. Arista pubescent, the longest hairs approximately equal to $1 / 2$ width of postpedicel. Palpus black. Thorax black, densely grey dusted. Acrostichals setulose in two rows, dorsocentrals $3+3$ (including scapular seta), intra-alars $1+2$, supra-alars $1+2,2$ postpronotals. Scutellum with a pair of strong discal and a pair of strong apical setae (Fig. 13). Legs greyish dusted. Femora of all legs black, only apex yellow; tibiae and tarsi of all legs yellow. Wing tinged with brownish; veins brownish. Vein $\mathrm{R}_{1}$ bare on apical third of dorsal surface. Abdomen black, densely grey dusted. Male sternite 4 rectangular, almost as long as wide. Male sternite 5, epandrium and surstyli as in Figs 90-92.

DISTRIBUTION. Russia: Altai, Arkhangelsk Oblast, Bashkiria, Buryatia, Chukotka, Karelia, Khabarovsk Krai, Komi, Krasnoyarsk Krai, Leningrad Oblast, Moscow Oblast, Murmansk Oblast, Novgorod Oblast, Novosibirsk Oblast, Primorsky Krai, Sakhalin Oblast, Saratov Oblast, Sverdlov Oblast, Tver' Oblast, Tyumen' Oblast, Yakutia, Yaroslavl' Oblast. — Europe, Belarus (first record), China [Sun, 1993: 440], North America.

\section{Cordilura remmi Elberg, 1972} Figs 8, 93-95.

remmi Elberg, 1972: 91 (Cordilura). Type-locality: "Sakhalin I., environment of South Sakhalinsk" (Russia, Sakhalin Oblast).
REMARK. This species was described on material from Amur Oblast and Sakhalin Oblast, Khabarovsk and Primorsky Krai [Elberg, 1972: 91], after recorded in Russia also from Jewish Autonomus Oblast by Ozerov \& Krivosheina [2014: 211].

MATERIAL EXAMINED. Amur Oblast: Blagoveshchensk $\left(50.280^{\circ} \mathrm{N} 127.535^{\circ} \mathrm{E}\right), 17$. VIII.1982, A. Leley (1 9 , ZISP); River Nyukzha $\left(56.574^{\circ} \mathrm{N} 121.652^{\circ} \mathrm{E}\right), 18 . \mathrm{VI} .1976$, Soboleva (2 우, IBSS); Komsomol'sk-na-Amure, Silinskiy Park $\left(50.573^{\circ} \mathrm{N} 137.039^{\circ} \mathrm{E}\right)$, 29.V.1985, Mutin (1 9 , IBSS); Zeya Town $\left(53.748^{\circ} \mathrm{N} 127.261^{\circ} \mathrm{E}\right)$, 26.VI., 3-12.VII.1978, 16-20.VII., 3-13.VIII., 1.IX.1981, 14.VIII.1982, A. Ozerov, A. Shatalkin (14 $0^{\top} \sigma^{7}, 16$ 우, ZMUM); Zeysky Nature Reserve, cordon " $34 \mathrm{~km}$ " $\left(53.989^{\circ} \mathrm{N} 127.073^{\circ} \mathrm{E}\right)$, 24.-30.VII.1981, 10 and 21.VII.1982, A.L. Ozerov, A. Shatalkin, M. Krivosheina, O. Gorbunov (3 $0^{7} \mathrm{O}^{7}, 7$ 우, ZMUM); Zeysky Nature Reserve, cordon " $52 \mathrm{~km}$ " $\left(54.087^{\circ} \mathrm{N} 126.871^{\circ} \mathrm{E}\right), 24$.VIII.1981, 12.VIII.1982, A.L. Ozerov, A. Shatalkin (1 OT, 2 o+ , ZMUM); 5-6 $\mathrm{km}$ above of Ekimchan $\left(53.085^{\circ} \mathrm{N} 132.974^{\circ} \mathrm{E}\right), 23$. VIII.2006, A.B. Ryvkin (1 9 , ZMUM); $52 \mathrm{~km} \mathrm{~N}$ of Zeya Town $\left(54.087^{\circ} \mathrm{N}, 126.871^{\circ} \mathrm{E}\right)$, 20.VII.1982, V. Zlobin (2 OP, ZISP); Bol'shoy Never $\left(53.978^{\circ} \mathrm{N}\right.$ $\left.124.150^{\circ} \mathrm{E}\right)$, 8.IX.1974, Gorodkov (2 OO, ZISP); Buryatia: Barguzinsky Nature Reserve (ca. $54.35^{\circ} \mathrm{N} 109.51^{\circ} \mathrm{E}$ ), 15 and 22.VII.1965, Negrobov ( $2 \mathrm{O}^{7} \mathrm{O}^{2}, 3$ 우, ZISP); Jewish Autonomus Oblast: Malyy Khingan ridge, River Dichun $\left(48.545^{\circ} \mathrm{N} 130.760^{\circ} \mathrm{E}\right), 15 . \mathrm{VII} .1979$, A. Shatalkin ( $1 \sigma^{7}$, ZMUM); Khabarovsk Krai: Ochotsk env. $\left(59.366^{\circ} \mathrm{N} 143.229^{\circ} \mathrm{E}\right), 27$. VIII.1987, Gorodkov (3 90 , ZISP); Khabarovsk $\left(48.426^{\circ} \mathrm{N} 135.118^{\circ} \mathrm{E}\right), 23 . \mathrm{VI} .1912$, Speshilova-Petelina (1 9 , ZISP); same place, 17.VI.1931, V. Pereleshina $\left(1 \sigma^{7}, 1\right.$, ZMUM); Khabarovsk env. (ca. $48.426^{\circ} \mathrm{N} 135.118^{\circ} \mathrm{E}$ ), $21-$ 22.VIII.1979, Nartshuk (1 ㅇ, ZISP); Primorsky Krai: $30 \mathrm{~km} \mathrm{~S}$ of Lazo, Benevskoe $\left(43.166^{\circ} \mathrm{N} 133.757^{\circ} \mathrm{E}\right), 16$.VIII.1986, A. Leley $(1$ $\sigma^{7}$, ZISP); $5 \mathrm{~km} \mathrm{~S}$ of Yakovlevka $\left(44.427^{\circ} \mathrm{N} 133.479^{\circ} \mathrm{E}\right)$, 29.VI.1.VII.1986, A. Leley ( 1 O $^{7}, 2$ 우, IBSS); Vladivostok, Morskoe kladbishche $\left(43.083^{\circ} \mathrm{N} 131.934^{\circ} \mathrm{E}\right), 13 . \mathrm{VIII} .1993$, Belokobyl'skiy (1 $0^{7}$, ZISP); Gorno-Taezhnaya station, Krivoy klyuch $\left(43.693^{\circ} \mathrm{N}\right.$ $\left.132.1525^{\circ} \mathrm{E}\right), 30$.VII. and 26.VIII.1980, M. Mikhaylovskaya (2 $0^{7} \sigma^{7}$, IBSS); Dal'nerechensk $2\left(45.926^{\circ} \mathrm{N} 133.740^{\circ} \mathrm{E}\right), 25 . \mathrm{VII} .1980, \mathrm{M}$. Mikhaylovskaya (1 $\mathrm{O}^{\top}$, IBSS); Kamenushka $\left(43.634^{\circ} \mathrm{N} 132.222^{\circ} \mathrm{E}\right)$, 21-30.VII., 19-20.VIII.1983, 25.VII., 4-26.VIII.1984, 4 and 17.VI.1985, 14-16.VII.1986, 9-26.VIII., 1-15.IX.1987, 31.VIII.3.IX.1988, 19.VIII.1989, A. Antropov, A.L. Ozerov, A. Shatalkin, S. Churkin (21 $\mathrm{O}^{7} \mathrm{O}^{7}, 15$ OP, ZMUM); same place, 7.VII. and 13.VIII. 1981, Mutin, Belova ( $1 \sigma^{7}, 1$ O., IBSS); Kedrovaya Pad' Nature Reserve $\left(43.104^{\circ} \mathrm{N} 131.512^{\circ} \mathrm{E}\right), 22$. VIII.1982, Romanova ( 1 , ZISP); same place, 29.VIII.1978, D. Shcherbakov (1 ○7, ZMUM); Kievka $\left(42.904^{\circ} \mathrm{N} 133.701^{\circ} \mathrm{E}\right), 2 . \mathrm{IX} .1980$, A. Shatalkin (1 9 , ZMUM); Lazovsky Nature Reserve, $10 \mathrm{~km} \mathrm{~W}$ of Preobrazhenie $\left(42.912^{\circ} \mathrm{N}\right.$ $\left.133.774^{\circ} \mathrm{E}\right), 16 . \mathrm{VIII} .1986$, A. Leley (1 $0^{7}$, ZISP); Bikin River, 22 $\mathrm{km}$ above of the mouth of the River Svetlovodnaya $\left(46.616^{\circ} \mathrm{N}\right.$ $\left.137.054^{\circ} \mathrm{E}\right), 14$.VIII.1980, V. Zlobin (2 9 ,, ZISP); River Bol'shaya Ussurka, $20 \mathrm{~km} \mathrm{NW}$ of Mel'nichnoe $\left(45.548^{\circ} \mathrm{N} 135.347^{\circ} \mathrm{E}\right)$, 2.VIII.1986, A. Leley (3 우, IBSS); Spassk $\left(44.600^{\circ} \mathrm{N} 132.816^{\circ} \mathrm{E}\right)$, 6-17.VI., 3-10.VII.1961, Zhelokhovtsev (1 ○ึ, 4 우, ZMUM); Suvorovo $\left(44.248^{\circ} \mathrm{N} 135.347^{\circ} \mathrm{E}\right)$, 17.VII.1972, A. Ponomarenko (1 $\mathrm{O}^{7}$, ZMUM); Sudzukhinsky [=Lazovsky] Nature Reserve, Tachingou $\left(43.023^{\circ} \mathrm{N} 134.136^{\circ} \mathrm{E}\right), 23$.VIII., 6.IX.1948, Gussakovskiy (2 +9 , ZMUM); Yakovlevka $\left(44.422^{\circ} \mathrm{N} 133.474^{\circ} \mathrm{E}\right), 24$ and 27.VII.1962, Nartshuk (2 $\mathrm{O}^{7} \mathrm{O}^{7}, 3$ 우, ZISP); Shkotovskoe plateau (ca. $43.458^{\circ} \mathrm{N}$ $\left.132.632^{\circ} \mathrm{E}\right)$, 25.VIII.1962, Nartshuk (1 $0^{7}, 1$, Z, ZISP); Gornotaezhnoe $\left(43.697^{\circ} \mathrm{N} 132.156^{\circ} \mathrm{E}\right), 29 . \mathrm{VII}$. and 5.VIII.1980, Afanas'eva (2 우, ZISP); same place, 1 and 15.VII.1980, V. Zlobin (2 우, ZISP); Ussuriyskiy Nature Reserve (ca. $43.635^{\circ} \mathrm{N} 132.311^{\circ} \mathrm{E}$ ), 14-17.VII. 1981, Ovchinnikova (1 $\mathrm{O}^{7}$, ZISP); Monakino $\left(43.392^{\circ} \mathrm{N} 133.433^{\circ} \mathrm{E}\right)$, 30.VIII.1962, Nartshuk (1 o, ZISP); Kamenushka (43.634ºN, $\left.132.222^{\circ} \mathrm{E}\right), 23$.VI.1980, Zlobin (1 +, ZISP); Sikhote-Alin Reserve, Blagodatnoe $\left(44.975^{\circ} \mathrm{N}\right.$ 136.526 $\left.\mathrm{E}\right), 13 . \mathrm{VII} .2018$, M. Sergeev (1 $\mathrm{O}^{7}$, ZMUM); Sakhalin Oblast: Kunashir I., $7 \mathrm{~km}$ N of Mendeleevo $\left(44.023^{\circ} \mathrm{N} 145.680^{\circ} \mathrm{E}\right), 22$. VIII.1975, Berezantsev (1 ㅇ, IBSS); Kunashir I., $7 \mathrm{~km} \mathrm{~S}$ of Lake Lagunnoe $\left(44.001^{\circ} \mathrm{N} 145.760^{\circ} \mathrm{E}\right), 12$ and 15.VIII.1989, Leley, Sidorenko (1 $\sigma^{7}, 1$ ㅇ, IBSS); Kunashir I., caldera of Golovnin Volcano $\left(43.843^{\circ} \mathrm{N} 145.505^{\circ} \mathrm{E}\right)$, 3.VIII.1989, Sidorenko (1 $\sigma^{7}, 1$ ㅇ, IBSS); Kunashir I., Stolbchatyy Cape 

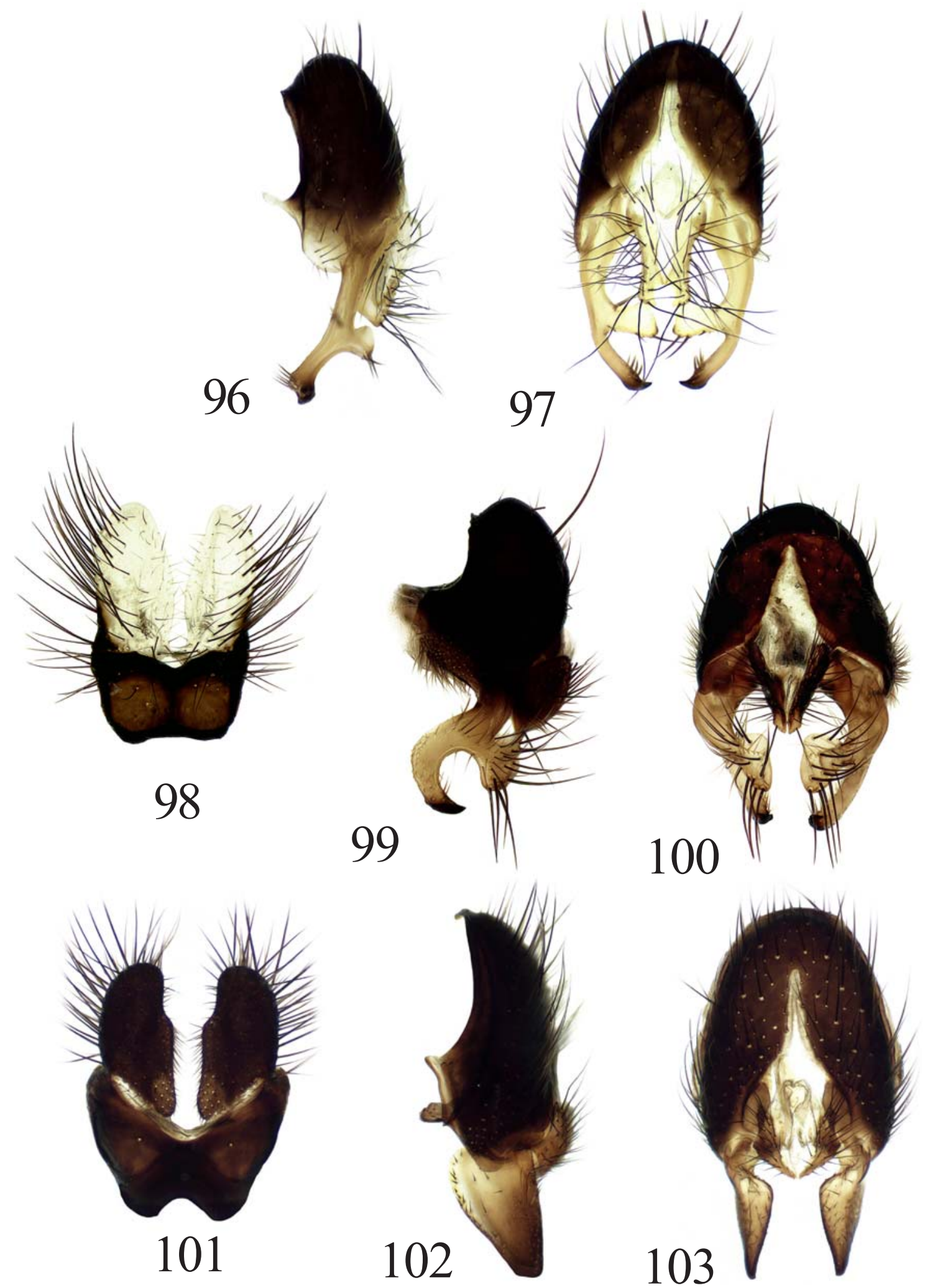

Figs 96-103. Cordilura remota Ozerov (96, 97), C. richterae Ozerov et Krivosheina (98-100) and C. rufimana (Meigen) (101-103): 98, 101 - male sternite 5; 96, 99, 102 - epandrium and surstyli, lateral view; 97, 100, 103 - same, dorsal view. 98-100 - after Ozerov \& Krivosheina, 2015, figs 2-4.

Рис. 96-103. Cordilura remota Ozerov (96, 97), C. richterae Ozerov et Krivosheina (98-100) и C. rufimana (Meigen) (101-103): 98, 101 - стернит 5 самца; 96, 99, 102 - эпандрий и сурстили, сбоку; 97, 100, 103 - то же, сверху. 98-100 - по Ozerov \& Krivosheina, 2015, figs $2-4$. 
$\left(44.023^{\circ} \mathrm{N} 145.678^{\circ} \mathrm{E}\right)$, 4.IX.1971, Nartshuk (2 oᄋ, ZISP); same place, 28.VII.1989, Sidorenko (1 $\sigma^{\top}$, IBSS); Kunashir I., YuzhnoKuril'sk env. $\left(44.029^{\circ} \mathrm{N} 145.860^{\circ} \mathrm{E}\right), 18-19$. VIII.1989, Leley, Sidorenko (1 $\sigma^{\top}, 1$ \%, IBSS); Kuril Islands, Shikotan I., Tserkovnaya Bay $\left(43.75^{\circ} \mathrm{N} 146.70^{\circ} \mathrm{E}\right), 10-30 . I X .2012$, Yu. Sundukov (2 0 , ZMUM); Sakhalin I., Pereput'e $\left(46.307^{\circ} \mathrm{N} 141.892^{\circ} \mathrm{E}\right), 22$.VII. 1953, N. Filippov (1 $\sigma^{7}$, ZMUM); Kunashir I., Mendeleev Volcano env. (43.961 $\left.{ }^{\circ} \mathrm{N} 145.729^{\circ} \mathrm{E}\right), 26$. VII.1985, S. Churkin ( 1 \% , ZMUM); Kunashir I., Kurilsky Nature Reserve, cordon Andreevsky $\left(43.54^{\circ} \mathrm{N}\right.$ $\left.145.37^{\circ} \mathrm{E}\right), 6-8$. VII.2014, I.A. Gomyranov (2 $\sigma^{7} \sigma^{7}, 1$ \% , ZMUM); same place, 8.VII.2014, T.V. Galinskaya (3 $\sigma^{7} \sigma^{7}, 3$ +o, ZMUM); Kunashir I., Tret'yakovo $\left(43.989^{\circ} \mathrm{N} 145.644^{\circ} \mathrm{E}\right)$, 8.VIII.1971, Nartshuk (1 $\sigma^{7}$, ZISP); same place, 10.VII.2085, S. Churkin (1 ZMUM); same place, 12.VII.2014, T.V. Galinskaya (2 ऽ $\sigma^{7}$, ZMUM); Kunashir I., Alekhino $\left(43.955^{\circ} \mathrm{N} 145.592^{\circ} \mathrm{E}\right), 31 . \mathrm{VIII}$ 1971, Nartshuk (1 9 , ZISP); same place, 28.VII.1973, Kasparyan (1 O', ZISP); Kunashir I., Yuzhno-Kuril'sk $\left(44.029^{\circ} \mathrm{N} 145.859^{\circ} \mathrm{E}\right)$, 12.VIII.1971, Nartshuk (1 $\sigma^{7}$, ZISP); Sakhalin I., Novo-Aleksandrovsk $\left(47.056^{\circ} \mathrm{N} 142.730^{\circ} \mathrm{E}\right), 18 . \mathrm{VII} .1961$, Krivolutskaya (1 $\sigma^{\top}$, IBSS); same place, 11.VII.1968, Nartshuk (1 9 , ZISP); Zabaykalsky Krai: the upper reaches of the River Ingoda, River Dzhila $\left(50.994^{\circ} \mathrm{N}\right.$ $\left.111.887^{\circ} \mathrm{E}\right)$, 4.VII.1947, Zhelokhovtsev (1 $\sigma^{7}$, ZMUM); Lesnoy $\left(51.730^{\circ} \mathrm{N} 113.071^{\circ} \mathrm{E}\right), 4$. VII.1971, V. Richter (1 q, ZISP).

DIAGNOSTIC DESCRIPTION. Male. Female. Body-length 6.2-9.8 mm. Head. Frontal vitta black with weak whitish reflection; fronto-orbital plate black, shining. Face and parafacial yellowish, with whitish reflection in male, and black, delicately grey dusted in female. Gena black in both sexes. Postcranium black completely, greyish dusted. 2 orbital and 3-4 frontal setae present. Antenna brownish in male; postpedicel approximately 4 times as long as wide. Antenna blackish in female; postpedicel approximately 3 times as long as wide. Arista pubescent or plumose, the longest hairs approximately equal to $1 / 2-3 / 4$ width of postpedicel. Palpus from dark yellow to black. Thorax black, delicately greyish dusted; scutum subshining. Acrostichals absent, dorsocentrals $2+3$ (including scapular seta), intra-alars absent, supraalars $0+2,1$ postpronotal. Scutellum with a pair of strong discal setae only; apical scutellar setae as setulae. Legs shining. Fore coxa white. Femora of all legs black; fore and mid tibiae, also tarsi of all legs yellow; hind tibia usually brownish. Wing tinged with strong brownish; veins blackish. Vein $\mathrm{R}_{1}$ setulose on apical third of dorsal surface. Abdomen black, subshining. Male sternite 4 rectangular, longer than wide. Male sternite 5, epandrium and surstyli as in Figs 93-95.

DISTRIBUTION. Russia: Amur Oblast, Buryatia, Jewish Autonomus Oblast, Khabarovsk Krai, Primorsky Krai, Sakhalin Oblast, Zabaykalsky Krai. — Japan [Iwasa, 2020: 477].

\section{Cordilura remota Ozerov, 1997} Figs 96, 97.

remota Ozerov, 1997: 1425 (Cordilura). Type-locality: Zeya Town (Russia, Amur Oblast).

REMARK. The species was described from Amur Oblast and Primorsky Krai [Ozerov, 1997: 1425].

MATERIAL EXAMINED. Amur Oblast: Zeya Town (53. $\left.748^{\circ} \mathrm{N} 127.261^{\circ} \mathrm{E}\right), 15$. VI.1981, A. Ozerov (1 paratype $\left.\sigma^{\top}, \mathrm{ZMUM}\right)$ Primorsky Krai: Ryazanovka (42.797 $\left.\mathrm{N} 131.244^{\circ} \mathrm{E}\right), 11$. VI.1989, A. Shatalkin (1 paratype $\left.\sigma^{7}, \mathrm{ZMUM}\right)$; Andreevka env. $\left(42.7^{\circ} \mathrm{N}\right.$ 131.1 $\left.{ }^{\circ} \mathrm{E}\right), 26-31$. VII.2018, N. Vikhrev (1 O', ZMUM).
DIAGNOSTIC DESCRIPTION. Male. Body-length $5.0-6.5 \mathrm{~mm}$. Head. Frontal vitta and fronto-orbital plate yellow. Face, parafacial and gena yellowish, with weak whitish reflection. Postcranium yellow in lower part and black in upper half. 2 orbital and 2 frontal setae present. Antenna pale yellow. Postpedicel approximately 2 times as long as wide. Arista plumose, the longest hairs approximately equal to the width of postpedicel. Palpus yellow. Thorax yellow in ground colour, but scutum black between postronotal lobes and intra-alar lines; mediotergite black. Acrostichals setulose in two rows, dorsocentrals $3+3$ (including scapular seta), intra-alars not developed, supra-alars $1+2,2$ postpronotals. Scutellum with a pair of strong discal setae only; apical scutellar setae as setulae. Legs shining, yellow. Wing tinged with brownish, with a distinct brown shadow apically; veins blackish. Vein $\mathrm{R}_{1}$ setulose on apical third of dorsal surface. Abdomen black, subshining. Male sternite 4 rectangular, longer than wide. Epandrium and surstyli as in Figs 96, 97.

Female unknown. Krai.

DISTRIBUTION. Russia: Amur Oblast, Primorsky

\section{Cordilura richterae Ozerov et Krivosheina, 2015} Figs 98-100.

richterae Ozerov, Krivosheina, 2015: 491 (Cordilura). Typelocality: Iturup I., Rybaki $\left(45.207^{\circ} \mathrm{N} 147.848^{\circ} \mathrm{E}\right)$ (Russia, Sakhalin Oblast).

REMARK. This species was known from the typelocality only.

MATERIAL EXAMINED. Sakhalin Oblast: Kunashir I., Dubovoe $\left(43.766^{\circ} \mathrm{N} 145.499^{\circ} \mathrm{E}\right), 10 . \mathrm{VI} .1973$, Ermolenko (2 $\bigcirc^{7} \sigma^{7}$, ZISP); Kunashir I., Lake Lagunnoe $\left(44.060^{\circ} \mathrm{N} 145.759^{\circ} \mathrm{E}\right), 18 . \mathrm{VI}$. 1968, Nartshuk (2 $\sigma^{\top} \sigma^{\top}$, ZISP).

DIAGNOSTIC DESCRIPTION. Male. Bodylength 4.8-7.2 mm. Head. Frontal vitta yellow, whitish dusted; fronto-orbital plate whitish dusted, yellow completely or blackish in upper half and yellow in lower half. Face, parafacial and gena yellow, whitish dusted. Postcranium black, delicatly greyish dusted. 2 orbital and 2-3 frontal setae present. Antenna yellow. Postpedicel approximately 2 times as long as wide. Arista yellow basally and black apically, plumose in basal half, the longest hairs approximately equal to the width of postpedicel. Palpus yellow. Thorax black, scutum subshining, thoracic sclerites greyish dusted. Acrostichals setulose in two rows, dorsocentrals $3+3$ (including short scapular seta), intra-alars $(0-1)+1$, supra-alars $1+2,2$ postpronotals. Scutellum with a pair of strong discal setae and a pair of apical setulae. Legs. Ground-colour yellow. Coxae of mid and hind legs black, mid and hind tibiae darkened. Wing tinged with brownish; veins pale brown. Vein $\mathrm{R}_{1}$ with 4-5 setulae of dorsal surface. Abdomen black, delicately greyish dusted. Sternite 4 trapezoid, slightly longer than wide. Sternite 5, epandrium and surstyli as in Figs $98-100$.

Female unknown.

DISTRIBUTION. Russia: Sakhalin Oblast. 

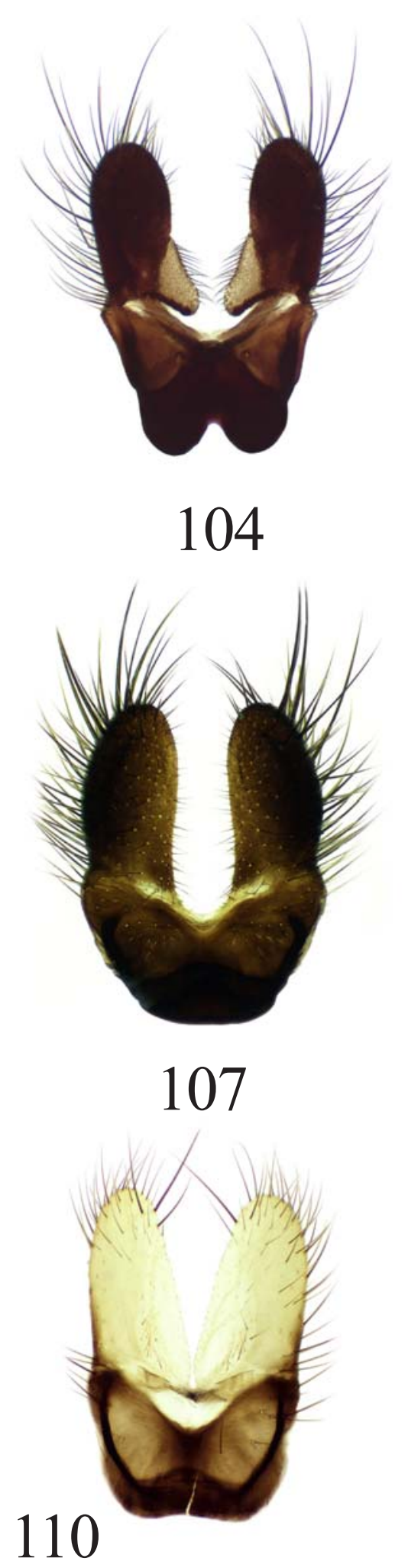
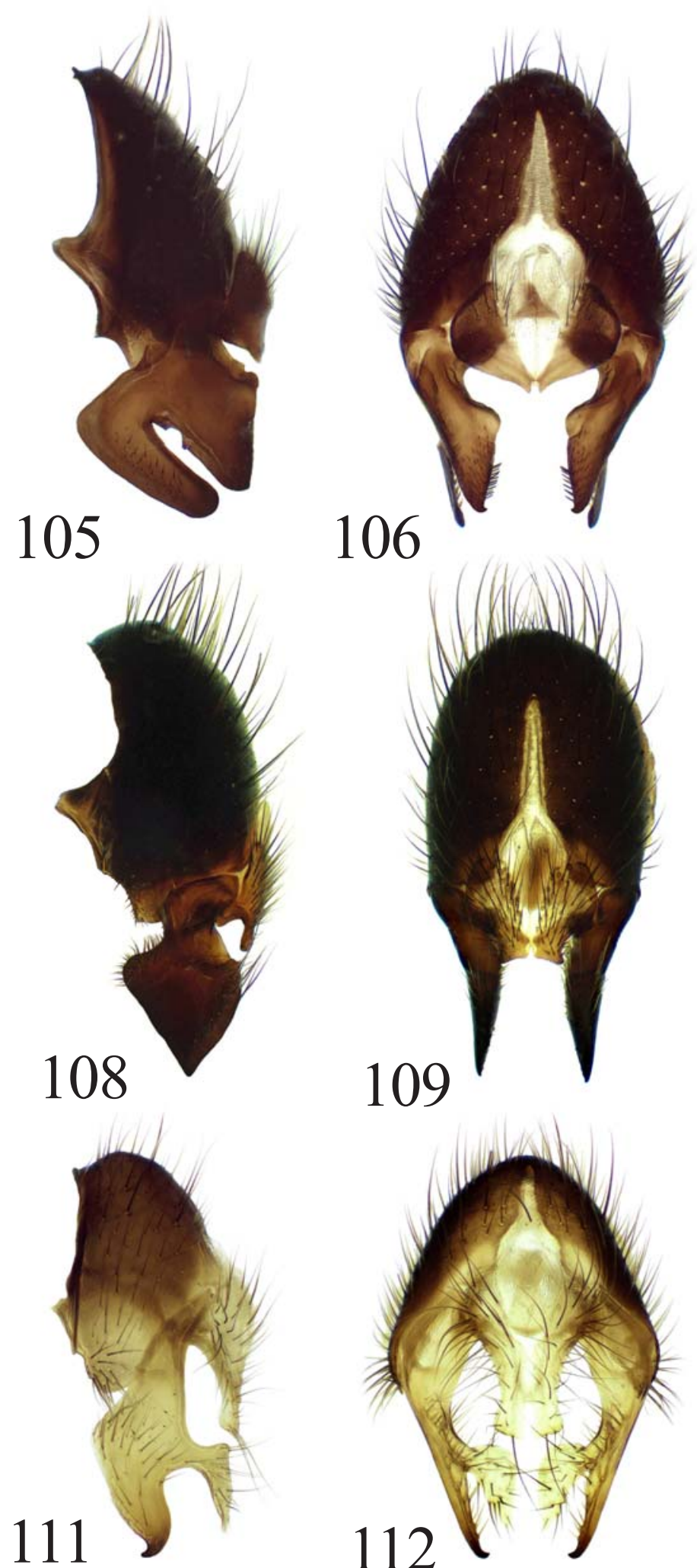
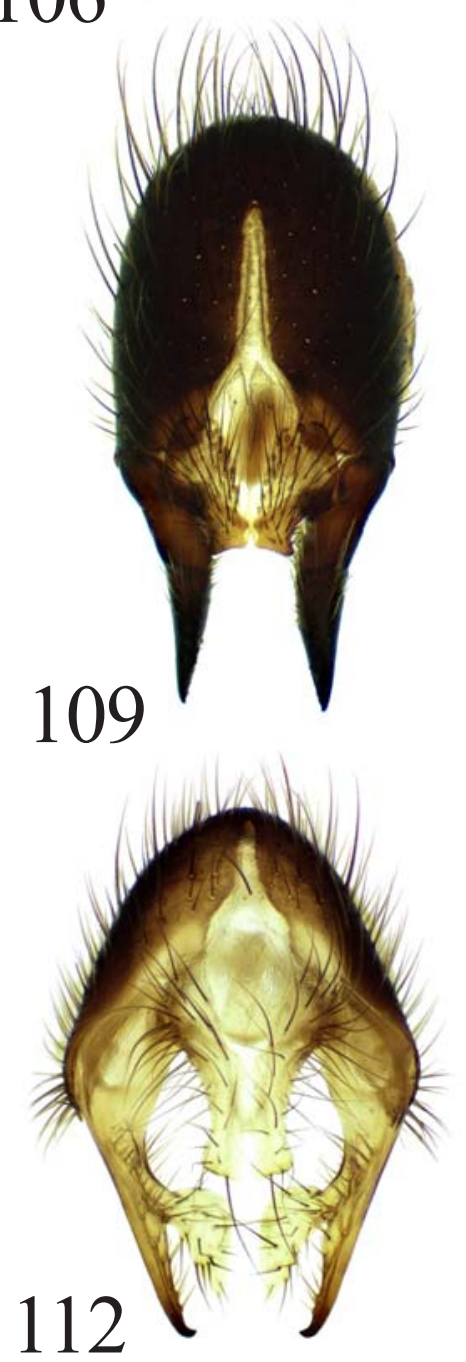

Figs 104-112. Cordilura rufipes (Meigen) (104-106), C. sagittifera Gorodkov (107-109) and C. shatalkini Ozerov (110-112): 104, 107,110 - male sternite 5; 105, 108,111 - epandrium and surstyli, lateral view; 106, 109, 112 - same, dorsal view. 105 - after Ozerov \& Krivosheina, 2014, fig. 15.

Pис. 104-112. Cordilura rufipes (Meigen) (104-106), C. sagittifera Gorodkov (107-109) и C. shatalkini Ozerov (110-112): 104, 107, 110 - стернит 5 самца; 105, 108, 111 - эпандрий и сурстили, сбоку; 106, 109, 112 - то же, сверху. 105 - по Ozerov \& Krivosheina, 2014, fig. 15. 
Cordilura rufimana (Meigen, 1826)

Figs 17, 101-103. given.

rufimana Meigen, 1826: 232 (Cordylura). Type-locality: not

incerta Zetterstedt, 1838: 690 (Anthomyza). Type-locality: "alpium Tornensium jugo.. .(Lapponia)" (Sweden).

tibialis Zetterstedt, 1838: 725 (Cordylura). Type-localities: "Lapponia Umensi... Lycksele et Åsele" (Sweden).

REMARK. Noted by Gorodkov [1970: 446, 1986: $15]$ for north of European part of Russia and north of Siberia without indicating specific locality; recorded from Yakutia [Bagachanova et al., 2016: 779].

MATERIAL EXAMINED. Altai: Kosh-Agach env., $\left(50.01^{\circ} \mathrm{N}\right.$ $\left.88.66^{\circ} \mathrm{E}\right), 1750 \mathrm{~m}, 2-4 . V I I .2016$, N. Vikhrev (1 $\sigma^{7}$, ZMUM); River Chirya [=Chiri] $\left(51.361^{\circ} \mathrm{N} 87.837^{\circ} \mathrm{E}\right)$, Lake Teletskoe, 30.VI.1909, Emel'yanov (1 $\mathrm{O}$, ZISP); Amur Oblast: Zeya Town $\left(53.748^{\circ} \mathrm{N}\right.$ 127.261 ${ }^{\circ}$ E), 12-17.VI.1978, 24.VII.1982, A. Shatalkin, A.L. Ozerov (1 $\sigma^{7}, 2$ 우, ZMUM); Khingansky Nature Reserve, $7 \mathrm{~km}$ E of Ukrainka $\left(49.477^{\circ} \mathrm{N} 129.666^{\circ} \mathrm{E}\right), 11 . \mathrm{VI} .1987$, A. Leley (1 + , IBSS); Bol'shoy Never $\left(53.978^{\circ} \mathrm{N} 124.150^{\circ} \mathrm{E}\right), 15 . \mathrm{VI} .1927$, Zakharzhevskiy (1 9 , ZISP); Arkhangelsk Oblast: Ust'-Kozhva $\left(65.144^{\circ} \mathrm{N}\right.$ $\left.57.002^{\circ} \mathrm{E}\right), 23$.VII.1908, Zhuravskiy (1 9 , ZISP); Nenetsky Nature Reserve, cordon "Bol'shoy Gusinets" $\left(68.175^{\circ} \mathrm{N} 53.645^{\circ} \mathrm{E}\right)$, 10.VII.2008, A.L. Ozerov (1 ㅇ, ZMUM); Buryatia: Selenduma $\left(50.916^{\circ} \mathrm{N} 106.239^{\circ} \mathrm{E}\right), 25 . \mathrm{VI} .1971, \mathrm{~V}$. Richter (2 90 , ZISP); Mondy env. (51.675 $\left.{ }^{\circ} \mathrm{N} 100.992^{\circ} \mathrm{E}\right), 15$.VII.1965, Gorodkov (1 $\left.\mathrm{O}^{\circ}, \mathrm{ZISP}\right)$ Chukotka: bank of the River Anadyr $\left(64.72^{\circ} \mathrm{N} 175.21^{\circ} \mathrm{E}\right), 25$.VI19.VII.2014, A. Barkalov (5 $\sigma^{7} \sigma^{\top}, 1$ \%, ZMUM and ISEA); Meynypil'gyno env. $\left(62.538^{\circ} \mathrm{N} 177.051^{\circ} \mathrm{E}\right), 15$.VII.2014, P.S. Tomkovich (1 $\left.\sigma^{\prime}, Z^{\prime} M U M\right)$; Bilibino $\left(68.058^{\circ} \mathrm{N} 166.446^{\circ} \mathrm{E}\right), 5 . \mathrm{VII} .1971$ Gorodkov ( $6 \sigma^{\top} \sigma^{\top}, 13$ 우, ZISP); Kamchatka Krai: Apuka env. $\left(60.443^{\circ} \mathrm{N} 169.602^{\circ} \mathrm{E}\right), 2$ and 3.VII.1959, Gorodkov (2 $\bigcirc^{7} \sigma^{7}$, ZISP); Karelia: Primorsky $\left(66.546^{\circ} \mathrm{N} 33.103^{\circ} \mathrm{E}\right), 30 . \mathrm{VI} ., 4$ and 6.VII.2010, A.L. Ozerov ( $2 \bigcirc^{\top} \sigma^{\top}, 1$ 19 , ZMUM); Komi: Sovetsky env. $\left(67.495^{\circ} \mathrm{N}\right.$ $\left.64.419^{\circ} \mathrm{E}\right), 20 . V I .2012$, S.V. Pestov (1 $\sigma^{7}$, ZMUM); Vil'gort $\left(60.560^{\circ} \mathrm{N} 56.465^{\circ} \mathrm{E}\right), 11 . \mathrm{VII} .1957$, Gabova (1 ${ }^{\circ}$, ZISP); Inta $\left(66.04^{\circ} \mathrm{N} 60.17^{\circ} \mathrm{E}\right), 5$.VIII.1985, Gorodkov (1 9 , ZISP); Ust'-Ukhta $\left(63.620^{\circ} \mathrm{N} 53.878^{\circ} \mathrm{E}\right)$, 4.VIII.1985, Gorodkov (1 ㅇ, ZISP); Krasnoyarsk Krai: Igarka $\left(67.457^{\circ} \mathrm{N} 86.598^{\circ} \mathrm{E}\right)$, River Yenisei, 1.VII.1967, Gorodkov (1 + , ZISP); Ergaki National Park $\left(52.84^{\circ} \mathrm{N} 93.25^{\circ} \mathrm{E}\right)$, 1450 m, 27-29.VI.2017, N. Vikhrev (3 $\sigma^{7} \sigma^{7}, 1$ ㅇ, ZMUM); Leningrad Oblast: Fan-der-Flit station $\left(58.641^{\circ} \mathrm{N} 29.693^{\circ} \mathrm{E}\right), 13$.VI.1925, A. Stackelberg (1 9 , ZISP); Rozhdestveno $\left(59.322^{\circ} \mathrm{N} 29.946^{\circ} \mathrm{E}\right)$, 4.VI.1956, A. Stackelberg (4 $\sigma^{\top} \sigma^{\top}, 1$ ㅇ, ZISP); Yashchera $\left(58.894^{\circ} \mathrm{N}\right.$ $\left.29.820^{\circ} \mathrm{E}\right), 23$.VII.1957, A. Stackelberg (2 $\sigma^{7} \sigma^{7}$, ZISP); Magadan Oblast: Sokol env. $\left(59.92^{\circ} \mathrm{N} 150.71^{\circ} \mathrm{E}\right), 11-19$.VII.2014, N. Vikhrev ( 1 ㅇ, ZMUM); Moscow Oblast: Dmitrov env. (56.316 $\left.{ }^{\circ} \mathrm{N} 37.725^{\circ} \mathrm{E}\right)$, 7.VI.2007, N. Vikhrev ( ${ }^{\circ}$, ZMUM); Murmansk Oblast: Lake Vud'yavr Basin (ca. $67.646^{\circ} \mathrm{N} 33.644^{\circ} \mathrm{E}$ ), 14.VII.1931, 27.VIII.1935, Fridolin (2 $\sigma^{7} \sigma^{7}$, ZISP); Novgorod Oblast: Ramen'e $\left(58.374^{\circ} \mathrm{N} 33.430^{\circ} \mathrm{E}\right)$, Okulovka env., 11.VI.1988, Gorodkov (1 $\sigma^{7}$ ZISP); Omsk Oblast: Irtysh env. $\left(54.99^{\circ} \mathrm{N} 73.32^{\circ} \mathrm{E}\right), 18-$ 22.VIII.2007, O. Kosterin (1 +, ZMUM); Sakhalin Oblast: Kunashir I., Mendeleev Volcano env. (43.961 $\left.{ }^{\circ} \mathrm{N} 145.729^{\circ} \mathrm{E}\right), 2$.VI.1985, S. Churkin (1 9 , ZMUM); Kunashir I., Tret'yakovo $\left(43.989^{\circ} \mathrm{N}\right.$ $\left.145.644^{\circ} \mathrm{E}\right), 15 . \mathrm{VI} .1968$, V. Richter (1 9 , ZISP); Kunashir I., Sernovodsk $\left(43.912^{\circ} \mathrm{N} 145.641^{\circ} \mathrm{E}\right), 30 . \mathrm{VI} .1968$, Nartshuk (1 ㅇ, ZISP); Smolensk Oblast: Smolenskoe poozer'e National Park $\left(55.511^{\circ} \mathrm{N}\right.$ $\left.31.839^{\circ} \mathrm{E}\right), 3$.VIII.1991, Zlobin (1 +, ZISP); Tyumen' Oblast: Seliyarovo $\left(61.467^{\circ} \mathrm{N} 70.731^{\circ} \mathrm{E}\right), 17-20$. VII.2010, K. Tomkovich (1 $\sigma^{7}, 1$ क, ZMUM); Obdorsk [=Salekhard] $\left(66.53^{\circ} \mathrm{N} 66.613^{\circ} \mathrm{E}\right), 15$ and 19.VII.1925, Fridolin (6 $\sigma^{\top}, 5$ o. ZISP); Saranpaul' $\left(64.257^{\circ} \mathrm{N} 60.917^{\circ} \mathrm{E}\right), 23 . \mathrm{VI} .1989$, Malozemov (1 + , ZISP); Ul'yanovsk Oblast: Baryshskaya sloboda $\left(54.583^{\circ} \mathrm{N} 46.791^{\circ} \mathrm{E}\right)$, 12.VI.1996, V.B. Isaeva (1 $\sigma^{7}$, ZMUM); Yakutia: River Yana, Stolby env. $\left(67.531^{\circ} \mathrm{N} 134.087^{\circ} \mathrm{E}\right), 26-28 . V I I .2008$, A. Ovchin-

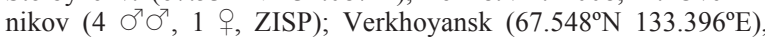
22-25.VII.1974, Gorodkov (10 $\sigma^{7} \sigma^{7}, 15$ 우, ZISP); Balagannakh

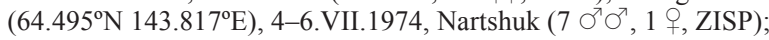
River Yana opposite of Verkhoyansk $\left(67.550^{\circ} \mathrm{N} 133.359^{\circ} \mathrm{E}\right), 21-$
23.VII.1974, Nartshuk (2 $\sigma^{7} \sigma^{7}, 2$ OO , ZISP); the mouth of the River Olekma $\left(60.366^{\circ} \mathrm{N} 120.667^{\circ} \mathrm{E}\right)$, 3.VIII.1974, Nartshuk (1 $\sigma^{7}$, ZISP); Batagay $\left(67.653^{\circ} \mathrm{N} 134.635^{\circ} \mathrm{E}\right), 21$. VII.1974, Gorodkov (1 ㅇ, ZISP); Zhatay env. $\left(62.162^{\circ} \mathrm{N} 129.834^{\circ} \mathrm{E}\right), 24$.VII.2002, Evdokarova (1 9 , ZISP); Zabaykalsky Krai: Darasun env. (51.200 $\left.\mathrm{N} 113.702^{\circ} \mathrm{E}\right)$, 26.VI.-7.VII.1949, N. Filippov (1 $\sigma^{7}, 1$ ㅇ, ZMUM).

DIAGNOSTIC DESCRIPTION. Male. Female. Body-length 4.8-7.9 mm. Head. Frontal vitta reddish yellow, matt; fronto-orbital plate black, greyish dusted. Face, parafacial and gena yellow with whitish reflection. Postcranium black, greyish dusted. 3 orbital and 3-4 frontal setae present. Antenna black. Postpedicel approximately 2 times as long as wide. Arista plumose, the longest hairs approximately equal to the width of postpedicel. Palpus black. Thorax black, greyish dusted. Acrostichals setulose in two rows, dorsocentrals $3+3$ (including scapular seta), intra-alars $1+2$, supraalars $1+2,2$ postpronotals. Scutellum with a pair of strong discal and a pair of strong apical setae. Legs delicately greyish dusted. Femora of all legs black; fore tibia and tarsi of all legs yellow; mid tibia from yellow to blackish; hind tibia black or dark brown. Wing tinged with brownish; veins brownish. Vein $\mathrm{R}_{1}$ bare on apical third of dorsal surface. Abdomen black, greyish dusted. Male sternite 4 rectangular, as long as wide (Fig. 17). Male sternite 5, epandrium and surstyli as in Figs 101-103.

DISTRIBUTION. Russia: Altai, Amur Oblast, Arkhangelsk Oblast, Buryatia, Chukotka, Kamchatka Krai, Karelia, Komi, Krasnoyarsk Krai, Leningrad Oblast, Magadan Oblast, Moscow Oblast, Novgorod Oblast, Omsk Oblast, Sakhalin Oblast, Smolensk Oblast, Tyumen' Oblast, Ul'yanovsk Oblast, Yakutia, Zabaykalsky Krai. - Europe, North America.

\section{Cordilura rufipes (Meigen, 1826)}

Figs 1, 3, 11, 14, 104-106. given.

rufipes Meigen, 1826: 232 (Cordylura). Type-locality: not pubera Linnaeus, 1758 sensu Fallén, 1810: 15 (Cordilura), misidentification.

REMARK. This species was recorded in Russia as widespread species by Gorodkov [1986: 15, as C. pubera Linnaeus, 1758] without specifying the place of collecting; recorded from Karelia [Humala, Polevoi, 2009: 71], from Saratov Oblast and Yaroslavl' Oblast [Ovchinnikov, 2004: 421], and from Yakutia [Bagachanova et al., 2016: 779].

MATERIAL EXAMINED. Altai: Lake Petukhovo $\left(52.25^{\circ} \mathrm{N}\right.$ 79.16 $\left.{ }^{\circ} \mathrm{E}\right)$, 20.VI.2009, O. Kosterin (1 $\sigma^{\top}$, ZMUM); Kosh-Agach env., $\left(50.01^{\circ} \mathrm{N} 88.66^{\circ} \mathrm{E}\right), 1750 \mathrm{~m}, 2-4 . V I I .2016$, N. Vikhrev $\left(1 \mathrm{O}^{2}\right.$, ZMUM); 10 km E of Cheke-Taman Pass, River Bol'shoy Il'gumen' $\left(50.613^{\circ} \mathrm{N} 86.469^{\circ} \mathrm{E}\right), 28$. VII.2006, A. Ovchinnikov $\left(1 \mathrm{O}^{7}, 1\right.$ q, ZISP); Artybash env. (51.796 $\left.{ }^{\circ} \mathrm{N} 87.265^{\circ} \mathrm{E}\right), 16 . \mathrm{VII} .2006$, A. Ovchinnikov (1 9 , ZISP); Turochak env. $\left(52.261^{\circ} \mathrm{N} 87.123^{\circ} \mathrm{E}\right), 17$. VII.2006, A. Ovchinnikov (1 9 , ZISP); same place, 18-19.VII.2006, Nartshuk $\left(1 \mathrm{O}^{7}\right.$, ZISP); River Chirya [=Chiri] $\left(51.361^{\circ} \mathrm{N} 87.837^{\circ} \mathrm{E}\right)$, Lake Teletskoe, 30.VI., 1 and 4.VII.1909, Emel'yanov (4 $0^{7} 0^{7}, 1$, ZISP); Arkhangelsk Oblast: Nar'yan-Mar env. $\left(67.627^{\circ} \mathrm{N} 53.000^{\circ} \mathrm{E}\right.$, $\left.67.631^{\circ} \mathrm{N} 52.985^{\circ} \mathrm{E}\right), 7-8$.VII.2008, N.E. Vikhrev, A.L. Ozerov (3 $0^{7} \sigma^{7}, 2$ 우, ZMUM); Nenetsky Nature Reserve, cordon "Bol'shoy Gusinets" $\left(68.175^{\circ} \mathrm{N} 53.645^{\circ} \mathrm{E}\right), 10$.VII.2008, A.L. Ozerov (1 O', 1 +, ZMUM); Solvychegodsk $\left(61.334^{\circ} \mathrm{N} 46.926^{\circ} \mathrm{E}, 61.327^{\circ} \mathrm{N}\right.$ 
46.926 $\left.{ }^{\circ} \mathrm{E}\right), 11$ and 17.VIII.2010, D. Gavryushin (2 oo , ZMUM); Bol'shoy Solovetskiy I. (65.078 $\left.{ }^{\circ} \mathrm{N}, 35.671^{\circ} \mathrm{E}\right), 12-29 . \mathrm{VII} .1997, \mathrm{~K}$. Tomkovich (1 $\Im^{7}$, ZMUM); airport Talagi $\left(64.597^{\circ} \mathrm{N} 40.709^{\circ} \mathrm{E}\right)$, 1.VIII.1978, Gorodkov (1 $\sigma^{7}, 1$ ㅇ, ZISP); Karpogory (ca. $64^{\circ} \mathrm{N}$ $44.45^{\circ} \mathrm{E}$ ), River Pinega, 10.VII.1996, Gorodkov (7 90 , ZISP); Velikovisochnoe $\left(67.255^{\circ} \mathrm{N} 52.032^{\circ} \mathrm{E}\right), 18$.VIII.1978, Gorodkov (1 O ZISP); Nar'yan-Mar $\left(67.636^{\circ} \mathrm{N} 53.031^{\circ} \mathrm{E}\right), 4,7$ and 20.VIII.1978, Gorodkov (6 $\sigma^{7} \sigma^{7}, 1$ \% ZISP); Nizhnyaya Pesha $\left(66.751^{\circ} \mathrm{N}\right.$ $\left.47.760^{\circ} \mathrm{E}\right)$, 22.VIII.1978, Gorodkov (1 +, ZISP); Solovetskie Islands $\left(65.080^{\circ} \mathrm{N} 35.686^{\circ} \mathrm{E}\right), 15 . \mathrm{VI} .1896$, Birulya (2 $\left.\sigma^{\top} \sigma^{\top}, \mathrm{ZISP}\right)$; Kargopol' (61.503 $\left.{ }^{\circ} \mathrm{N} 38.971^{\circ} \mathrm{E}\right), 13$ and 16.VII.1982, Gorodkov (2 $\sigma^{7} \sigma^{7}, 4$ Oᄋ, ZISP); Bashkiria: Irgizly $\left(52.960^{\circ} \mathrm{N} 57.023^{\circ} \mathrm{E}\right)$ 12.VI.1899, Yakobson \& Shmidt ( $1 \mathrm{O}^{7}$, ZISP); Abzakovo $\left(53.82^{\circ} \mathrm{N}\right.$, $\left.58.62^{\circ} \mathrm{E}\right), 500 \mathrm{~m}, 15-19 . V I .2020$, N. Vikhrev (2 $\Im^{7} \sigma^{7}$, ZMUM); Buryatia: Barguzinsky Nature Reserve (ca. $54.35^{\circ} \mathrm{N} 109.51^{\circ} \mathrm{E}$ ), 30.VII.1965, Negrobov ( 1 , ZISP); Chelyabinsk Oblast: Taganay $\left(55.277^{\circ} \mathrm{N} 59.795^{\circ} \mathrm{E}\right), 18-24 . \mathrm{VII} .2008, \mathrm{~K}$. Tomkovich ( 1 +, ZMUM); Crimea: (ca. $44.483^{\circ} \mathrm{N} 33.866^{\circ} \mathrm{E}$ ), "from Karakau to Yayly", Vidgal'm (4 $\bigcirc^{7} \circlearrowleft^{7}$, ZISP); Irkutsk Oblast: Listvyanka $\left(51.863^{\circ} \mathrm{N}\right.$ $\left.104.866^{\circ} \mathrm{E}\right), 24 . V I .1965$, Negrobov (2 0 , , ZISP); Novochunka $\left(56.114^{\circ} \mathrm{N} 99.273^{\circ} \mathrm{E}\right), 5 . \mathrm{VI} .1957$, Gorodkov (2 $\sigma^{7} \sigma^{7}$, ZISP); Kabardino-Balkaria: Terskol $\left(43.252^{\circ} \mathrm{N} 42.521^{\circ} \mathrm{E}\right), 2400 \mathrm{~m}$, 17.VI.1972, V. Richter (1 $\sigma^{7}$, ZISP); Karelia: Primorskiy $\left(66.546^{\circ} \mathrm{N}\right.$ 33.103 ${ }^{\circ}$ E), 3-6.VII.2010, A.L. Ozerov (4 $\sigma^{\top} \sigma^{7}, 10$ OO , ZMUM); Sumskoy posad $\left(64.238^{\circ} \mathrm{N} 35.408^{\circ} \mathrm{E}\right)$, 27.VII.1996, Gorodkov (1 , , ZISP); Kizhi $\left(62.084^{\circ} \mathrm{N} 35.211^{\circ} \mathrm{E}\right), 19 . \mathrm{VI} .1979$, Gorodkov (1 ${ }^{7}$, 1 o, ZISP); Komi: Vorkuta $\left(67.5^{\circ} \mathrm{N} 64.0^{\circ} \mathrm{E}\right), 19-25$. VII.2010, N. Vikhrev (4 o⿱, ZMUM); $3 \mathrm{~km} \mathrm{~N}$ of Ust-Tsilma (ca. $65.45^{\circ} \mathrm{N}$ $\left.52.11^{\circ} \mathrm{E}\right), 10$. VIII.1978, Gorodkov (1 $\sigma^{\top}$, ZISP); Inta $\left(66.04^{\circ} \mathrm{N}\right.$ $\left.60.17^{\circ} \mathrm{E}\right), 1-7$. VII.1961, Gabova (1 $0^{\top}, 5$ oㅇ, ZISP); Shchel'yabozh

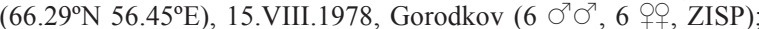
Shchel'yayur $\left(65.33^{\circ} \mathrm{N} 53.43^{\circ} \mathrm{E}\right), 12$.VIII.1978, Gorodkov (3 $\sigma^{7} \sigma^{7}$, 3 우, ZISP); Krasnodar Krai: Teberdinsky Nature Reserve (ca. $\left.43.405^{\circ} \mathrm{N} 41.721^{\circ} \mathrm{E}\right), 6 . V I I .1960$, Gorodkov (10 $\sigma^{7} \sigma^{7}, 5$ 우, ZISP); Krasnoyarsk Krai: "Stolby" $\left(55.962^{\circ} \mathrm{N} 92.750^{\circ} \mathrm{E}, 55.970^{\circ} \mathrm{N}\right.$ 92.742 ${ }^{\circ}$ E), 31.VII.2009, 19.VI.2011, K. Tomkovich (1 o', 2 우우, ZMUM); Dudinka $\left(69.404^{\circ} \mathrm{N} 86.182^{\circ} \mathrm{E}\right)$, 3.VII.1967, Gorodkov (1 $\sigma^{7}$, ZISP); Igarka $\left(67.457^{\circ} \mathrm{N} 86.598^{\circ} \mathrm{E}\right)$, River Yenisei, 30.VI.1967, Gorodkov ( 2 OO, ZISP); Ergaki National Park $\left(52.84^{\circ} \mathrm{N} 93.25^{\circ} \mathrm{E}\right)$ 1450 m, 27-29.VI.2017, N. Vikhrev (8 $\sigma^{7} \sigma^{7}, 2$ 우, ZMUM); Kursk Oblast: Zorino $\left(51.172^{\circ} \mathrm{N} 36.353^{\circ} \mathrm{E}\right), 14 . \mathrm{V} .2008$, K. Tomkovich (2 $\sigma^{7} \sigma^{7}, 1$, ZMUM); Leningrad Oblast: Bol'shoy Berezovyy I. (60.3 $\left.{ }^{\circ} \mathrm{N} 28.62^{\circ} \mathrm{E}\right), 31 . \mathrm{V}$. and 1.VI.1981, Kandybina (2 우, ZISP); Gatshina $\left(59.56^{\circ} \mathrm{N} 30.13^{\circ} \mathrm{E}\right), 10$ and $11 . \mathrm{VI} .1940, \mathrm{~A}$. Stackelberg (2 $\sigma^{\top} \mathrm{O}^{\top}$, ZISP); Lebyazh'ya $\left(59.963^{\circ} \mathrm{N} 29.421^{\circ} \mathrm{E}\right)$, 11.V.1897, Bianki (1 $\sigma^{7}, 1$ \%, ZISP); same place, 11.VI.1899, Chekini (1 q, ZISP); Ligovo $\left(60.223^{\circ} \mathrm{N} 31.797^{\circ} \mathrm{E}\right), 29 . \mathrm{V} .1903$, 15.VI.1904, 3.VIII.1911, G. Yakobson (3 $\sigma^{\top} \sigma^{\top}$, ZISP); Sablino (ca. $\left.59.63^{\circ} \mathrm{N} 30.76^{\circ} \mathrm{E}\right), 29$. V.1922, Fridman (1 \%, ZISP); Tolmachevo $\left(58.856^{\circ} \mathrm{N} 29.895^{\circ} \mathrm{E}\right), 11 . V I .1935$, Rohdendorf (1 $\sigma^{\circ}$, ZISP); same place, 27.VIII.1957, Nartshuk (1 $\sigma^{\top}$, ZISP); Shuvalovo $\left(60.047^{\circ} \mathrm{N}\right.$ $\left.30.283^{\circ} \mathrm{E}\right), 6-14 . V .1897,1 . \mathrm{VII} .1915$, Yakobson $\left(2 \bigcirc^{\top} \sigma^{7}, 6\right.$ 우, ZISP); Yashchera $\left(58.894^{\circ} \mathrm{N} 29.820^{\circ} \mathrm{E}\right), 24 . \mathrm{VI} .1956,14$ 19.VI.1957, 23.VII.1957, 5.VIII.1957, 12-25.VI.1958, 5.VIII.1958, A. Stackelberg ( $8 \bigcirc^{\top} \bigcirc^{\top}, 2$, + , ZISP); Mordovia: Mordovsky Nature Reserve, River Pushta $\left(54.72^{\circ} \mathrm{N}, 43.23^{\circ} \mathrm{E}\right), 18-21 . \mathrm{V} .2020, \mathrm{~N}$. Vikhrev (2 $\bigcirc^{7} \sigma^{7}$, ZMUM); Mordovsky Nature Reserve, River Pushta $\left(54.71^{\circ} \mathrm{N}, 43.22^{\circ} \mathrm{E}\right), 8-12 . V I .2020, \mathrm{~N}$. Vikhrev (1 $\sigma^{7}$, ZMUM); Mordovsky Nature Reserve, River Pushta $\left(54.77090^{\circ} \mathrm{N}, 43.2133^{\circ} \mathrm{E}\right)$, 18.V.2020, M. Esin (2 $\sigma^{7} \sigma^{7}, 1$ \%, ZMUM); Moscow and Moscow Oblast: $35 \mathrm{~km}$ NNE of Moscow $\left(56.200^{\circ} \mathrm{N} 37.833^{\circ} \mathrm{E}\right), 26 \mathrm{VI} .1993$, A.L. Ozerov (1 $\sigma^{7}$, ZMUM); Abramtsevo $\left(56.230^{\circ} \mathrm{N} 37.956^{\circ} \mathrm{E}\right)$, 31.V.1953, 9.VI.1958, E.S. Smirnov (1 $\sigma^{7}, 1$ \%, ZMUM); Burtsevo $\left(55.975^{\circ} \mathrm{N} 35.586^{\circ} \mathrm{E}\right), 2-3 . V I .2006$, A.L. Ozerov (1 $\sigma^{7}, \mathrm{ZMUM}^{\circ}$; Golitsyno $\left(55.649^{\circ} \mathrm{N} 37.011^{\circ} \mathrm{E}\right), 27 . \mathrm{V} .1978,3 . V .1981$, A. Shatalkin (1 Or, 1 q, ZMUM); Gorenki $\left(56.211^{\circ} \mathrm{N} 37.868^{\circ} \mathrm{E}\right)$, 5.VI.1944, E.S Smirnov (1 + , ZMUM); Dmitrov env. $\left(56.316^{\circ} \mathrm{N} 37.725^{\circ} \mathrm{E}\right)$,

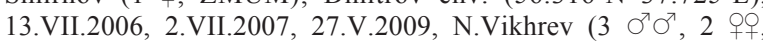
ZMUM); Naro-Fominsk $\left(55.357^{\circ} \mathrm{N} 36.736^{\circ} \mathrm{E}\right), 30$.V.2007, D. Gavryushin (2 $\sigma^{7} \sigma^{7}$, ZMUM); Poyarkovo $\left(56.009^{\circ} \mathrm{N} 37.328^{\circ} \mathrm{E}\right)$, 29.V.1999, A. Gusakov (2 $\sigma^{7} \sigma^{7}$, ZMUM); Stepan'kovo $\left(55.004^{\circ} \mathrm{N}\right.$ $35.624^{\circ} \mathrm{E}$ ), 3.VI.2006, A.L. Ozerov (3 $\mathrm{O}^{7} \mathrm{\sigma}^{7}$, ZMUM); Ozhigovo $\left(55.450^{\circ} \mathrm{N} 38.877^{\circ} \mathrm{E}\right)$, 3.VI.2010, D. Gavryushin (1 今, ZMUM);
Strogino $\left(55.793^{\circ} \mathrm{N} 37.410^{\circ} \mathrm{E}\right), 30 . \mathrm{V} .2015$, N.Vikhrev (1 $\sigma^{7}$, ZMUM); Izmaylovo $\left(55.787^{\circ} \mathrm{N} 37.836^{\circ} \mathrm{E}\right), 11 . V .2015$, A.L. Ozerov (1 9 , ZMUM); Yakshino env. $\left(55.920^{\circ} \mathrm{N} 35.573^{\circ} \mathrm{E}\right), 15 . \mathrm{V} .2015$, A.L. Ozerov (1 $\sigma^{7}$, ZMUM); Sofrino $\left(56.149^{\circ} \mathrm{N} 37.932^{\circ} \mathrm{E}\right)$, 24.VI.1946, Rohdendorf (1 $\sigma^{7}$, ZISP); Bittsa $\left(55.641^{\circ} \mathrm{N} 37.570^{\circ} \mathrm{E}\right)$, 15.VI.1936, B. Rohdendorf ( $1 \sigma^{7}$, ZISP); Murmansk Oblast: Pu-

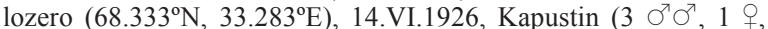
ZISP); Murmansk, Kola Bay $\left(68.951^{\circ} \mathrm{N} 33.033^{\circ} \mathrm{E}\right)$, 3.VII.1924, Kapustin (1 $\sigma^{7}, 1$, ZISP); Nizhegorod Oblast: Dzerzhinsk $\left(56.21^{\circ} \mathrm{N} 43.62^{\circ} \mathrm{E}\right), 16$. VIII.2009, N. Dvoretskaya (1 9, ZMUM); North Ossetia - Alania: Alagir env. (43.015 $\left.\mathrm{N} 44.224^{\circ} \mathrm{E}\right)$, 30.VI.1990, A. Shatalkin (1 $\sigma^{7}$, ZMUM); Bakhty-Laparyrag ridge $\left(42.938^{\circ} \mathrm{N} 44.287^{\circ} \mathrm{E}\right), 1770 \mathrm{~m}, 13 . \mathrm{VII} .1989,30 . \mathrm{VI} .1990$, A.L. Ozerov, A. Shatalkin (3 $\sigma^{7}, 1$, ZMUM); Novgorod Oblast: Volkhovmost $\left(59.069^{\circ} \mathrm{N} 31.765^{\circ} \mathrm{E}\right), 22 . \mathrm{VI} .1975$, Gorodkov (2 $\mathrm{O}^{7} \mathrm{\sigma}^{\top}, 2$ 우, ZISP); Novgorod, Spas-Nereditsa $\left(58.497^{\circ} \mathrm{N} 31.311^{\circ} \mathrm{E}\right), 22 . \mathrm{VI} .1975$, Gorodkov ( 3 +, ZISP); Novosibirsk Oblast: Novosibirsk $\left(54.842^{\circ} \mathrm{N}\right.$ 83.114 $\left.{ }^{\circ} \mathrm{E}\right), 14$. VI.2008, O. Kosterin (7 90, ZMUM); Novosibirsk, Academgorodok $\left(54.87^{\circ} \mathrm{N} 83.05^{\circ} \mathrm{E}\right), 18-19$. VI.2016, N. Vikhrev (1 9 , ZMUM); Omsk Oblast: Achairsky monastery $\left(54.669^{\circ} \mathrm{N}\right.$ 73.819 ${ }^{\circ}$ E), 22.VI.2008, O. Kosterin (1 o, ZMUM); Primorsky Krai: Kamenushka $\left(43.634^{\circ} \mathrm{N} 132.222^{\circ} \mathrm{E}\right), 14$.VII.1983, A. Shatalkin (1 $\sigma^{7}$, ZMUM); Pskov Oblast: Kharlamova Gora $\left(58.713^{\circ} \mathrm{N} 28.713^{\circ} \mathrm{E}\right), 26 . \mathrm{V}$. and 2.VI.1891, Pleske (1 フ, 2 우, ZISP); Dubets Lake $\left(56.966^{\circ} \mathrm{N} 30.006^{\circ} \mathrm{E}\right), 2$.VI.1915, N. Kuznetsov (1 $\sigma^{7}$, ZISP); Ryazan' Oblast: River Ranova $\left(53.715^{\circ} \mathrm{N}\right.$ $\left.39.926^{\circ} \mathrm{E}\right)$, 6.VI.2012, K. Tomkovich (1 $\sigma^{7}$, ZMUM); Kasimov $\left(54.94^{\circ} \mathrm{N} 41.34^{\circ} \mathrm{E}\right), 25 . V I I .2013$, N. Vikhrev (1 $\sigma^{7}$, ZMUM); Kasimov env. (54.969 $\left.{ }^{\circ} \mathrm{N} 41.327^{\circ} \mathrm{E}\right), 21-24 . V I I .2013, \mathrm{~N}$. Vikhrev (2 $\sigma^{\top} \sigma^{\top}, 1$ + 1 ZMUM); Sakhalin Oblast: Shikotan I., Tserkovnaya Bay $\left(43.75^{\circ} \mathrm{N}, 146.70^{\circ} \mathrm{E}\right), 27-31$.VIII.2012, Yu. Sundukov (1 $\sigma^{7}$, ZMUM); Samara Oblast: Bakhilova Polyana $\left(53.436^{\circ} \mathrm{N} 49.663^{\circ} \mathrm{E}\right)$, 24.VI.2006, A. Ovchinnikov (1 $\sigma^{\top}, 2$ 2 , Z ZISP); Seredysh I., Lake Kol'chuzhino $\left(53.452^{\circ} \mathrm{N} 49.719^{\circ} \mathrm{E}\right), 26 . V I .2006$, E.P. Nartshuk, A. Ovchinnikov (1 $\sigma^{\top}, 3$ 우, ZISP); Smolensk Oblast: Smolenskoe poozer'e National Park $\left(55.511^{\circ} \mathrm{N} 31.839^{\circ} \mathrm{E}\right), 8$.VI.1992, Zlobin (1 9 , ZISP); Sverdlovsk Oblast: Polevskoy env., Kosoy brod $\left(56.479^{\circ} \mathrm{N}\right.$ $\left.60.329^{\circ} \mathrm{E}\right)$, 5.VII.1981, Zinov'ev (1 $\sigma^{7}$, ZISP); Tyumen' Oblast: Surgut $\left(61.259^{\circ} \mathrm{N} 73.418^{\circ} \mathrm{E}\right), 25$ and $26 . \mathrm{VII} .1977$, Gorodkov (8 $\mathrm{O}^{\top} \mathrm{O}^{\top}, 9$ 우, ZISP); Tobol'sk (58.208º $\left.68.326^{\circ} \mathrm{E}\right)$, VI.1908, B.M. Zhitkov (1 + , ZMUM); Labytnangi $\left(66.657^{\circ} \mathrm{N} 66.391^{\circ} \mathrm{E}\right)$, 24.VII.1973, L. Zimina (1 $\sigma^{7}$, ZMUM); $\left(61.73^{\circ} \mathrm{N} 71.03^{\circ} \mathrm{E}\right)$, 21.VII.2010, K. Tomkovich (2 $\sigma^{\top} \sigma^{\top}$, ZMUM); Shapsha env. $\left(61.085^{\circ} \mathrm{N} 69.458^{\circ} \mathrm{E}\right), 14-16 . \mathrm{VII} .2010, \mathrm{~K}$. Tomkovich (1 9, ZMUM); Mukhino $\left(60.89^{\circ} \mathrm{N} 68.70^{\circ} \mathrm{E}\right), 7-13 . \mathrm{VIII} .2010, \mathrm{~K}$. Tomkovich $(1$, ZMUM); Labytnangi $\left(66.661^{\circ} \mathrm{N} 66.394^{\circ} \mathrm{E}\right), 5$ and 20.VIII.1978, Sychevskaya (2 $\sigma^{7} \sigma^{7}$, ZISP); same place, 14.VII.1985, Gorodkov (1 $\sigma^{7}, 2$ 20 , ZISP); Lake Varcha-ty $\left(66.107^{\circ} \mathrm{N} 63.987^{\circ} \mathrm{E}\right), 29$. VIII. and 2.IX.1925, Fridolin (1 $\sigma^{\top}, 3$ O 9 , ZISP); Neroyka (ca. $64.57^{\circ} \mathrm{N}$

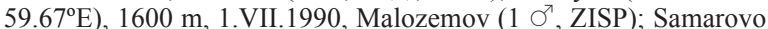
$\left(60.968^{\circ} \mathrm{N} 69.041^{\circ} \mathrm{E}\right)$ [now is a part of Khanty-Mansiysk], 24.VI.1930, N. Plotnikov (1 ア ' ZISP); Saranpaul' $\left(64.257^{\circ} \mathrm{N}\right.$ $60.917^{\circ} \mathrm{E}$ ), 30.VII.1990, Malozemov (1 + , ZISP); Vladimir Oblast: $\left(56.06^{\circ} \mathrm{N} 40.46^{\circ} \mathrm{E}\right), 5 . \mathrm{VI} .2011, \mathrm{~N}$. Vikhrev (1 $\left.\sigma^{\top}, \mathrm{ZMUM}\right)$; Vladimir env. $\left(56.05^{\circ} \mathrm{N} 40.41^{\circ} \mathrm{E}\right), 16 . \mathrm{VI} .2013$, N. Vikhrev $\left(1 \sigma^{7}, 1\right.$ +, ZMUM); Vorohezh Oblast: Georgiu-Dezh [=Liski] $\left(50.979^{\circ} \mathrm{N}\right.$ 39.55 $\left.1^{\circ} \mathrm{E}\right)$, 6.IX.1969, Skuf' in (1 $\sigma^{\top}$, ZISP); Usmanskiy Bor $\left(51.911^{\circ} \mathrm{N} 39.562^{\circ} \mathrm{E}\right), 16 . \mathrm{V}$. and 1.VI.1962, Skuf'in (2 $\sigma^{7} \sigma^{7}$, ZISP); Khopersky Nature Reserve (ca. $51.191^{\circ} \mathrm{N} 41.706^{\circ} \mathrm{E}$ ), 7.VII.1962, Skuf' in (1 $\sigma^{7}$, ZISP); Varvarino $\left(51.209^{\circ} \mathrm{N} 41.719^{\circ} \mathrm{E}\right), 10$.VIII.1969, Dorovskaya (1 9 , ZISP); Yakutia: Yakutsk env. $\left(62.042^{\circ} \mathrm{N}\right.$ 129.708 E), 16-17.VI.1962, Zhelokhovtsev (1 ○', 1 \%, ZMUM); same place, 24.VI.1957, Gorodkov (1 ○, 1 , ZISP); Arylakh $\left(62.385^{\circ} \mathrm{N} 114.350^{\circ} \mathrm{E}\right), 8 . \mathrm{VII} .1987$, Zlobin (1 $\sigma^{7}$, ZISP); Mikhaylovka, $60 \mathrm{~km} \mathrm{NE}$ of Amga $\left(61.214^{\circ} \mathrm{N} 132.681^{\circ} \mathrm{E}\right), 17 . V I I .1987$, Bagachanova (1 $\mathrm{O}^{7}$, ZISP); Khaptagay $\left(61.787^{\circ} \mathrm{N} 129.795^{\circ} \mathrm{E}\right)$, 7.VI.1974 and 14.VI.1975, Bagachanova (1 O', 1 q, ZISP); Oktemtsy env. $\left(61.672^{\circ} \mathrm{N} 129.417^{\circ} \mathrm{E}\right)$, 5.VI. 1977, Maksimova (2 $\sigma^{7} \sigma^{7}, 1$, ZISP); Yaroslavl' Oblast: Berditsyno $\left(57.455^{\circ} \mathrm{N} 40.107^{\circ} \mathrm{E}\right)$, 2.VII.1908, A. Yakovlev (1 $\sigma^{7}$, ZISP).

ADDITIONAL MATERIAL EXAMINED. Georgia: Bakuriani $\left(41.752^{\circ} \mathrm{N} 43.530^{\circ} \mathrm{E}\right), 2200 \mathrm{~m}, 23 . \mathrm{V} .1972$, Zinov'eva (1

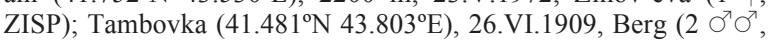





Figs 113-121. Cordilura sibirica Gorodkov (113-115), C. sidorenkoi Ozerov et Krivosheina (116-118) and C. sifneri Ozerov (119121): 113, 116, 119 - male sternite 5; 114, 117, 120 — epandrium and surstyli, lateral view; $115,118,121$ — same, dorsal view. 116 118- after Ozerov \& Krivosheina, 2012b, figs 1, 3, 4.

Рис. 113-121. Cordilura sibirica Gorodkov (113-115), C. sidorenkoi Ozerov et Krivosheina (116-118) и C. sifneri Ozerov (119121): 113, 116, 119 - стернит 5 самца; $114,117,120$ - эпандрий и сурстили, сбоку; 115, 118, 121 - то же, сверху. 116-118 - по Ozerov \& Krivosheina, 2012b, figs 1, 3, 4. 
ZISP); Kazakhstan: Bosaga $\left(47.88^{\circ} \mathrm{N} 72.956^{\circ} \mathrm{E}\right)$, 23.V.1959, Grunin (3 $O^{7} \sigma^{7}, 2$ 웅, ZISP).

DIAGNOSTIC DESCRIPTION. Male (Fig. 1). Female. Body-length 5.8-9.6 mm. Head. Frontal vitta from reddish yellow to blackish, with whitish reflection; fronto-orbital plate black, greyish dusted. Face and parafacial yellow with whitish reflection. Gena yellow or brownish. Postcranium black, grey dusted. 3 orbital and 2-4 frontal setae present. Antenna black. Postpedicel approximately 2 times as long as wide. Arista plumose, the longest hairs approximately equal to the width of postpedicel (Fig. 3). Palpus black. Thorax black, greyish dusted. Acrostichals setulose in two rows, dorsocentrals $3+3$ (including scapular seta), intra-alars $1+(1-2)$, supra-alars $1+2,2$ postpronotals. Scutellum with a pair of strong discal and a pair of strong apical setae. Legs greyish dusted. Fore coxa black. Femora of all legs black; tibiae and tarsi of all legs yellow; tarsomeres 2-4 of all or only fore legs with black spot basally (Fig. 11). Wing tinged with yellowish; veins brownish. Vein $\mathrm{R}_{1}$ setulose on apical third of dorsal surface. Abdomen black, greyish dusted. Male sternite 4 trapezoid, approximately 2 times as long as wide (Fig. 14). Male sternite 5, epandrium and surstyli as in Figs 104-106.

DISTRIBUTION. Russia: Altai, Arkhangelsk Oblast, Bashkiria, Buryatia, Chelyabinsk Oblast, Crimea, Irkutsk Oblast, Kabardino-Balkaria, Karelia, Komi, Krasnodar Krai, Krasnoyarsk Krai, Kursk Oblast, Leningrad Oblast, Mordovia, Moscow Oblast, Murmansk Oblast, Nizhegorod Oblast, North Ossetia - Alania, Novgorod Oblast, Novosibirsk Oblast, Omsk Oblast, Primorsky Krai, Pskov Oblast, Ryazan’ Oblast, Sakhalin Oblast, Samara Oblast, Smolensk Oblast, Sverdlovsk Oblast, Tyumen' Oblast, Vladimir Oblast, Vorohezh Oblast, Yakutia, Yaroslavl' Oblast. - Europe, Georgia (first record), Kazakhstan (first record), Iran [Khaghaninia \& Gharajedaghi, 2014: 390], Mongolia [Gorodkov, 1986: 15], China [Sun, 1993: 440].

\section{Cordilura sagittifera Gorodkov, 1974} Figs 107-109.

sagittifera Gorodkov, 1974: 388 (Cordilura). Type-locality: 6

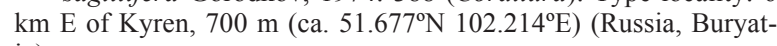
ia).

REMARK. This species was described on material from Buryatia and Irkutsk Oblast [Gorodkov, 1974: 388] and later registered from Amur Oblast and Primorsky Krai [Ozerov, Krivosheina, 2014: 212], also from Yakutia [Bagachanova et al., 2016: 779].

MATERIAL EXAMINED. Altai: Lake Kolyvanskoe $\left(51.363^{\circ} \mathrm{N}\right.$ 82.191 ${ }^{\circ} \mathrm{E}$ ), 8.IX.2007, O. Kosterin (1 $\sigma^{7}$, ZMUM); Amur Oblast: Zeysky Nature Reserve, cordon "52 km" $\left(54.087^{\circ} \mathrm{N} 126.871^{\circ} \mathrm{E}\right)$, 7.VIII.1981, A. Ozerov (1 O', 1 q, ZMUM); Bashkiria: Beloretsk $\left(54.051^{\circ} \mathrm{N} 58.268^{\circ} \mathrm{E}\right), 12$. VIII.2012, D. Gavryushin (1 $\left.\sigma^{7}, \mathrm{ZMUM}\right)$ Buryatia: Khargun, $6 \mathrm{~km} \mathrm{E}$ of Kyren $\left(51.677^{\circ} \mathrm{N}, 102.214^{\circ} \mathrm{E}\right)$, Sayan Mountains, 700 m, 11.VII.1965, Gorodkov (holotype $\sigma^{7}, 1$ paratype + , ZISP); Novosibirsk Oblast: Novosibirsk env. $\left(54.842^{\circ} \mathrm{N}\right.$

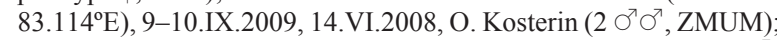
same place $\left(54.75^{\circ} \mathrm{N} 83.01^{\circ} \mathrm{E}\right), 11 . \mathrm{V} .2008$, O. Kosterin $\left(1 \sigma^{7}\right.$, ZMUM); Primorsky Krai: Lake Khanka $\left(45.06^{\circ} \mathrm{N} 131.99^{\circ} \mathrm{E}\right), 4$ 6.VII.2014, N. Vikhrev (1 $\sigma^{7}$, ZMUM); Yakutia: Alagar-Khoy, 18 $\mathrm{km} \mathrm{N}$ of Tyungyulyu $\left(62.346^{\circ} \mathrm{N} 130.649^{\circ} \mathrm{E}\right), 21$.VII.1985, Bagachanova (1 $\sigma^{7}$, ZISP); Mikhaylovka, $60 \mathrm{~km} \mathrm{NE}$ of Amga $\left(61.214^{\circ} \mathrm{N}\right.$ $\left.132.681^{\circ} \mathrm{E}\right), 7 . V I I .1987$, Bagachanova (3 90 , ZISP); Yakutsk env.

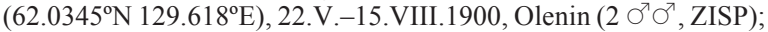
Zabaykalsky Krai: Ulyatuy $\left(51.168^{\circ} \mathrm{N} 116.227^{\circ} \mathrm{E}\right)$, 16.VIII.2016, A. Medvedev (1 9 , ZMUM).

DIAGNOSTIC DESCRIPTION. Male. Female. Body-length 6.0-7.6 mm. Head. Frontal vitta black, with whitish reflection; fronto-orbital plate black, greyish dusted. Face and parafacial yellowish with whitish reflection. Gena blackish. Postcranium black, grey dusted. 3 orbital and 2-3 frontal setae present. Antenna black. Postpedicel approximately 2 times as long as wide. Arista plumose, the longest hairs approximately equal to the width of postpedicel. Palpus black. Thorax black, delicately grey dusted. Acrostichals setulose in two rows, dorsocentrals $3+3$ (including small scapular seta), intra-alars $(0-1)+2$, supra-alars $1+2,2$ postpronotals. Scutellum with a pair of strong discal setae only; apical scutellar setae as setulae or absent. Legs greyish dusted. Fore coxa black; femora of all legs black; tibiae and tarsi of all legs yellow. Wing tinged with brownish; veins blackish. Vein $\mathrm{R}_{1}$ setulose on apical third of dorsal surface. Abdomen black, delicately grey dusted. Male sternite 4 rectangular, slightly wider than long. Male sternite 5, epandrium and surstyli as in Figs 107-109.

DISTRIBUTION. Russia: Altai, Amur Oblast, Bashkiria, Buryatia, Novosibirsk Oblast, Primorsky Krai, Yakutia, Zabaykalsky Krai. - Mongolia [Gorodkov, 1974: 388].

\section{Cordilura shatalkini Ozerov, 1997} Figs 20, 110-112.

shatalkini Ozerov, 1997: 1426 (Cordilura). Type-locality: Ryazanovka (Russia, Primorsky Krai).

REMARK. This species was registered in Russia from Primorsky Krai [Ozerov, 1997: 1426; Ozerov, Krivosheina, 2014: 212].

MATERIAL EXAMINED. Primorsky Krai: Shkotovo env., River Maykhe [=Artemovka] $\left(43.345^{\circ} \mathrm{N} 132.305^{\circ} \mathrm{E}\right), 4 . \mathrm{VI} .1927$, Stackelberg (1 \%', ZISP).

DIAGNOSTIC DESCRIPTION. Male. Female. Body-length 6.2-7.0 mm. Head. Frontal vitta and fronto-orbital plate yellow. Face, parafacial and gena yellowish, with weak whitish reflection. Postcranium yellow in lower part and black in upper half. 2 orbital and 2 frontal setae present. Antenna pale yellow. Postpedicel approximately 2 times as long as wide. Arista plumose, the longest hairs approximately equal to the width of postpedicel. Palpus yellow. Thorax black completely or pleural sclerites yellow, only mediotergite black, also anepimeron and katatergite partly blackish. Acrostichals setulose in two rows, dorsocentrals $3+3$ (including scapular seta), intra-alars $(0-1)+(0-1)$, supra-alars $1+2,2$ postpronotals. Scutellum with a pair of strong discal setae only; apical scutellar setae as setulae. Legs shining, yellow. Wing tinged with brownish, with a distinct brown shadow apically (Fig. 20); veins blackish. Vein $\mathrm{R}_{1}$ setulose on apical third of dorsal surface. Abdomen black, subshining. Male sternite 4 trapezoid, approximately 2 


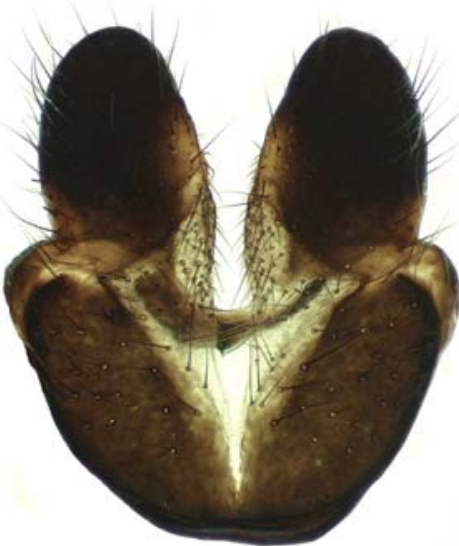

122

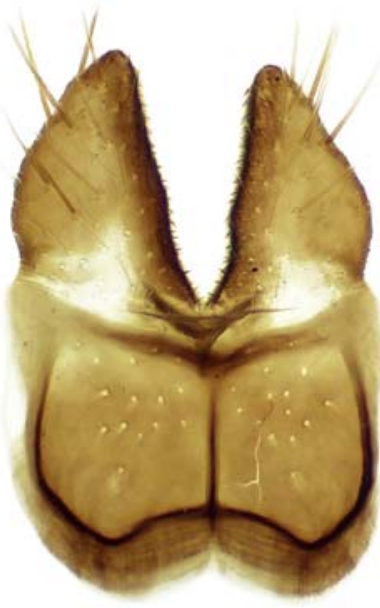

125

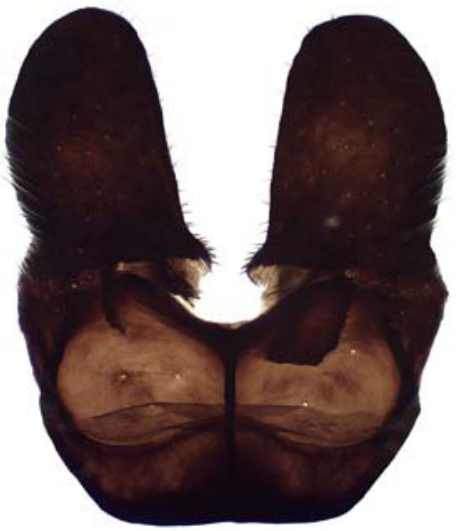

128
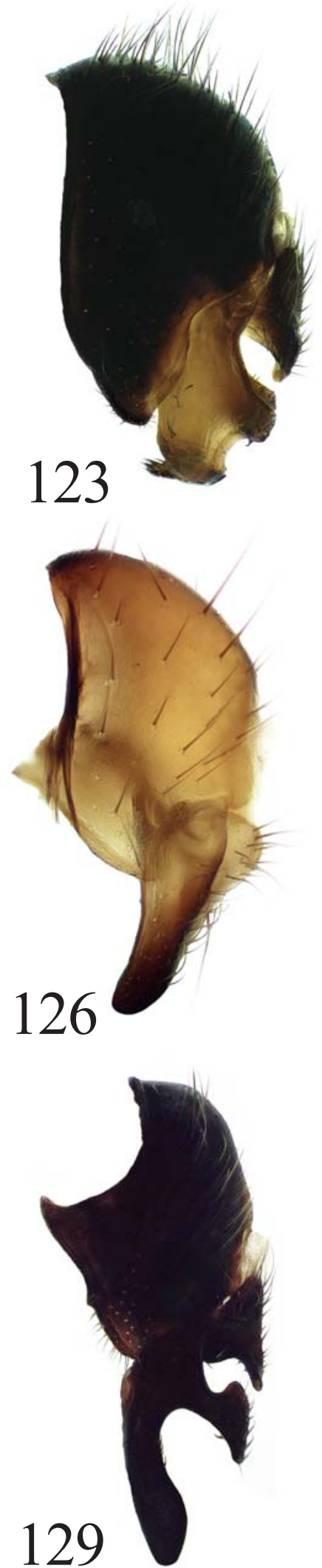

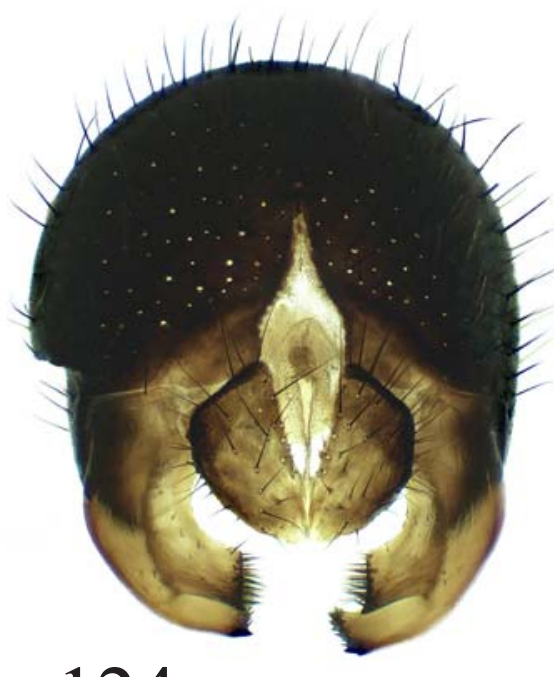

124
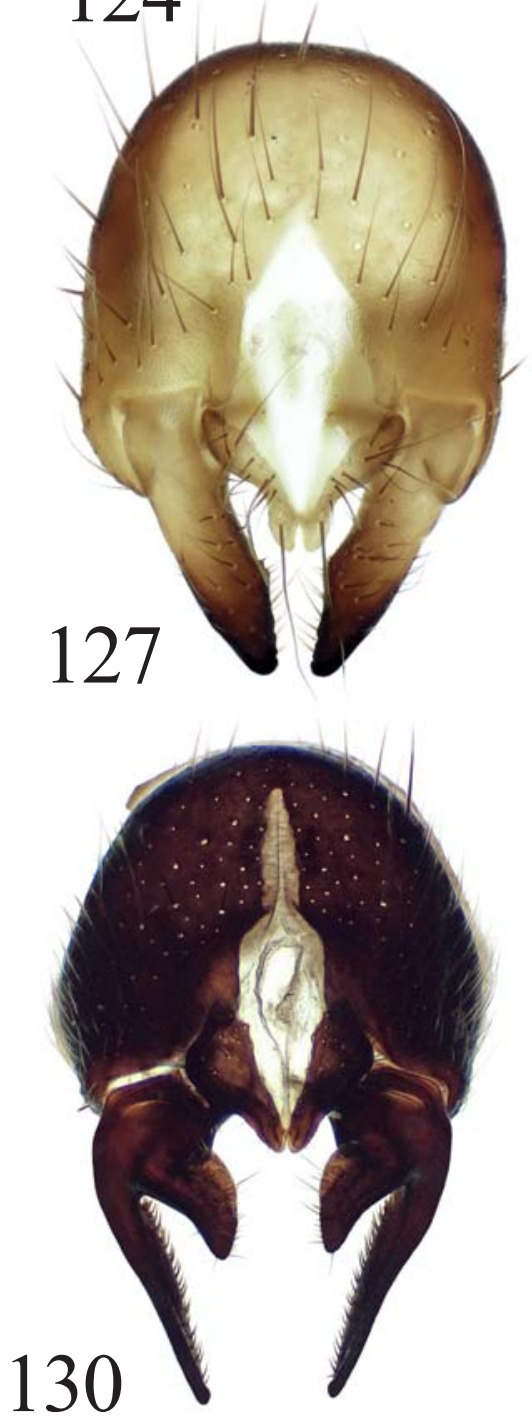

Figs 122-130. Cordilura socialis (Becker) (122-124), C. tartariana Ozerov (125-127) and C. ustulata (Zetterstedt) (128-130): 122, 125, 128 - male sternite 5; 123, 126, 129 - epandrium and surstyli, lateral view; 124, 127, 130 - same, dorsal view.

Рис. 122-130. Cordilura socialis (Becker) (122-124), C. tartariana Ozerov (125-127) и C. ustulata (Zetterstedt) (128-130): 122, 125,128 - стернит 5 самца; 123, 126, 129 - эпандрий и сурстили, сбоку; 124, 127, 130 - то же, сверху. 
times as long as wide. Male sternite 5, epandrium and surstyli as in Figs 110-112.

DISTRIBUTION. Russia: Primorsky Krai.

\section{Cordilura sibirica Gorodkov, 1974} Figs 113-115.

sibirica Gorodkov, 1974: 388 (Cordilura). Type-locality: 6 $\mathrm{km} \mathrm{E}$ of Kyren, $700 \mathrm{~m}$ (ca. $51.677^{\circ} \mathrm{N} 102.214^{\circ} \mathrm{E}$ ) (Russia, Buryatia).

REMARK. This species is known from a single male specimen [Gorodkov, 1974: 388].

MATERIAL EXAMINED. Buryatia: Khargun, $6 \mathrm{~km}$ E of Kyren $\left(51.677^{\circ} \mathrm{N} 102.214^{\circ} \mathrm{E}\right)$, Sayan Mountains, $700 \mathrm{~m}$, 11.VII.1965, Gorodkov (holotype $\sigma^{r}$, ZISP).

DIAGNOSTIC DESCRIPTION. Male. Body-length $7.5 \mathrm{~mm}$. Head. Frontal vitta blackish, with whitish reflection; fronto-orbital plate black, with whitish reflection. Face yellowish with whitish reflection. Parafacial brownish, gena blackish. Postcranium black, grey dusted. 3 orbital and 3-4 frontal setae present. Antenna black. Postpedicel approximately 2 times as long as wide. Arista plumose, the longest hairs approximately equal to the width of postpedicel. Palpus black. Thorax black, grey dusted. Acrostichals setulose in two rows, dorsocentrals $3+3$ (including scapular seta), intra-alars $0+(1-2)$, supra-alars $1+2,2$ postpronotals. Scutellum with a pair of strong discal setae only; apical scutellar setae as setulae or absent. Legs greyish dusted. Fore coxa black; femora of all legs black; tibiae and tarsi of all legs yellow. Wing tinged with brownish; veins blackish. Vein $\mathrm{R}_{1}$ setulose on apical third of dorsal surface. Abdomen black, grey dusted. Male sternite 4 rectangular, slightly longer than wide. Male sternite 5, epandrium and surstyli as in Figs 113-115.

Female unknown.

DISTRIBUTION. Russia: Buryatia.

Cordilura sidorenkoi Ozerov et Krivosheina, 2012 Figs 116-118.

sidorenkoi Ozerov, Krivosheina, 2012b: 2 (Cordilura). Typelocality: Lazovsky Nature Reserve $\left(43.005^{\circ} \mathrm{N}, 134.123^{\circ} \mathrm{E}\right)$ (Russia, Primorsky Krai).

REMARK. The species is known from the typelocality only.

MATERIAL EXAMINED. Primorsky Krai: Lazovsky Nature Reserve $\left(43.005^{\circ} \mathrm{N} 134.123^{\circ} \mathrm{E}\right), 22-23 . V .2007, \mathrm{~V}$. Sidorenko (holotype $\sigma^{\top}$, ZMUM).

DIAGNOSTIC DESCRIPTION. Male. Body-length $6.8 \mathrm{~mm}$. Head. Frontal vitta and orbital plate yellow, whitish dusted. Face, parafacial and gena yellow, whitish dusted. Postcranium blackish in dorsal half (with black setae and setulae) and yellowish in ventral half (covered with yellow hairs). 2 orbital and 2 frontal setae present. Antenna yellow. Postpedicel approximately 2 times as long as wide. Arista yellow basally and black apically, plumose in basal half. Palpus yellow. Scutum black, greyish dusted, with yellowish median stripe between dorsocentral setae along entire scutum and with lateral yellowish stripes along each supra-alar lines. Pleura greyish dusted, mostly yellow, but anepimeron, katatergite, anatergite and mediotergite black, and meron blackish. Acrostichals setulose usually in two rows, dorsocentrals $3+3$ (including scapular seta), intra-alars $1+0$, supra-alars $1+1,2$ postpronotals. Scutellum with a pair of strong discal setae and a pair of apical setulae. Legs entirely yellow. Wing tinged with yellowish, with a distinct brown shadow apically; veins brown. Vein $\mathrm{R}_{1}$ setulose on apical third of dorsal surface. Abdomen black in ground colour. Sternite 4 trapezoid, slightly longer than wide. Sternite 5, epandrium and surstyli as in Figs 116-118.

Female unknown.

DISTRIBUTION. Russia: Primorsky Krai.

Cordilura sifneri Ozerov, 2007

Figs 9, 119-121.

sifneri Ozerov, 2007b: 126 (Cordilura). Type-locality: $40 \mathrm{~km}$ SE of Ussuriysk (Russia, Primorsky Krai).

REMARK. Recorded from Primorsky Krai of Russia only [Ozerov, 2007b: 126; Ozerov, Krivosheina, 2014: 212].

MATERIAL EXAMINED. Primorsky Krai: Lazovsky Nature Reserve, cordon “Amerika” (43.283N 134.044E), 13-18.VII.1986, A.L. Ozerov ( 8 + 9 , ZMUM); Kedrovaya Pad' Nature Reserve (43.104 $\left.{ }^{\circ} \mathrm{N} 131.512^{\circ} \mathrm{E}\right), 8-16 . V I .2007$, A. Ovchinnikov $\left(1 \sigma^{7}, 1\right.$ ㅇ, ZISP); Ussuriysky Nature Reserve (ca. $\left.43.635^{\circ} \mathrm{N} 132.311^{\circ} \mathrm{E}\right), 13-$ 14.VI.1993, Belokobyl'skiy (1 $\sigma^{7}$, ZISP); $30 \mathrm{~km} \mathrm{SE} \mathrm{of} \mathrm{Ussuriysk,}$ Ussuriysky Nature Reserve (43.635 $\left.\mathrm{N} 132.311^{\circ} \mathrm{E}\right), 11-13 . \mathrm{VI} .1993$,

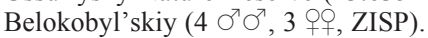

DIAGNOSTIC DESCRIPTION. Male, female. Body-length 4.8-6.5 mm. Head. Frontal vitta yellow, matt; fronto-orbital plate blackish, greyish dusted. Face, parafacial and gena yellowish white. Postcranium greyish dusted, yellow in upper half and whitish in lower half. 2 orbital and 2-3 frontal setae present. Antenna yellow. Postpedicel approximately 2.5 times as long as wide. Arista nearly bare. Palpus pale yellow. Thorax yellow; scutum with two dark rows, each exterior from dorsocentral lines; mediotergite with two dark stripes on margins; anepisternum with dark band on upper margin in some specimens. Acrostichals setulose in two rows, dorsocentrals $3+3$ (including scapular seta), intra-alars $1+(1-2)$, supra-alars $1+2,2$ postpronotals. Scutellum with a pair of strong discal setae and a pair of apical setulae. Legs yellow; usually tarsomere 3 in apical half, tarsomeres 4 and 5 of mid leg, and tarsomere 5 of hind leg dark brownish in male; all tarsi yellow in female. Wing tinged with light brownish; veins brownish. Vein $\mathrm{R}_{1}$ setulose on apical third or half of dorsal surface. Abdomen yellow in ground color, slightly darkened along border between tergites in male; more darkened in female. Male sternite 4 rectangular, longer than wide. Male sternite 5, epandrium and surstyli as in Figs 119-121.

DISTRIBUTION. Russia: Primorsky Krai.

Cordilura socialis (Becker, 1894) Figs 122-124.

socialis Becker, 1894: 90 (Cordylura). Type-locality: “C. pudica. Zett. Dipt. Sc. V. 1998, 3 ["Suecia meridionali... in Scania... 
ad Hafniam" (Sweden, Denmark)]; Rond., Prodr. VII, 14, 4.["unicum exemplar germanicum" (Germany)]".

freyi Hackman, 1956: 16 (Cordylura). Type-locality: "Ks: Kuusamo, Tavajärvi" (Finland).

REMARK. Noted for North of European part of Russia without indicating specific locality [Gorodkov, 1986: 15].

MATERIAL EXAMINED. Arkhangelsk Oblast: Nar'yan-Mar $\left(67.631^{\circ} \mathrm{N}, 52.985^{\circ} \mathrm{E}\right), 12$. VII.2008, N.E. Vikhrev (1 ㅇ, ZMUM) Moscow Oblast: Burtsevo env. $\left(55.995^{\circ} \mathrm{N} 35.617^{\circ} \mathrm{E}, 55.978^{\circ} \mathrm{N}\right.$

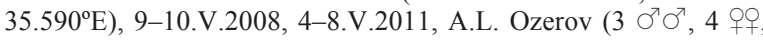
ZMUM); Andreevskoe env. $\left(55.978^{\circ} \mathrm{N} 35.588^{\circ} \mathrm{E}, 55.995^{\circ} \mathrm{N}\right.$ $35.617^{\circ} \mathrm{E}$ ), 14 and 20.V.2007, A.L. Ozerov (4 $\sigma^{7} \sigma^{7}$, ZMUM).

DIAGNOSTIC DESCRIPTION. Male. Female. Body-length 5.4-6.9 mm. Head. Frontal vitta dark brown, matt; fronto-orbital plate black, greyish dusted. Face, parafacial and gena yellowish with whitish reflection. Postcranium black, greyish dusted. 3 orbital and 4-5 frontal setae present. Scapus and pedicel dark brown; postpedicel black approximately 1.5-2 times as long as wide. Arista pubescent, the longest hairs approximately equal to $1 / 3-1 / 2$ width of postpedicel. Palpus black. Thorax black, delicately greyish dusted. Acrostichals setulose in two rows, dorsocentrals (3$4)+3$ (including scapular seta), intra-alars 1+2, supraalars $1+2,2$ postpronotals. Scutellum with a pair of strong discal and a pair of strong apical setae. Legs delicately greyish dusted. Femora black, tibiae and tarsi of all legs yellow. Wing tinged with light brownish; veins brownish. Vein $\mathrm{R}_{1}$ bare on apical third of dorsal surface. Abdomen black, greyish dusted. Male sternite 4 rectangular, wider than long. Male sternite 5, epandrium and surstyli as in Figs 122-124.

DISTRIBUTION. Russia: Arkhangelsk Oblast, Moscow Oblast. - Europe.

\section{Cordilura tartariana Ozerov, 2007} Figs $125-127$.

tartariana Ozerov, 2007b: 126 (Cordilura). Type-locality: Zeya Town (Russia, Amur Oblast).

REMARK. The species is known from Amur Oblast, Khabarovsk Krai and Primorsky Krai [Ozerov, 2007: 126; Ozerov, Krivosheina, 2014: 212].

MATERIAL EXAMINED. Amur Oblast: Zeya Town $\left(53.748^{\circ} \mathrm{N} 127.261^{\circ} \mathrm{E}\right), 23$. VI.-8.VII.1978, 27-31.VII.1982, A. Shatalkin (5 $\sigma^{\top} \sigma^{7}, 8$ 우, ZMUM); Zeysky Nature Reserve, cordon

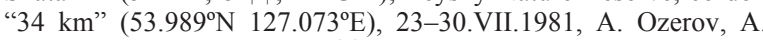
Shatalkin, O. Gorbunov (11 OO, ZMUM); Zeysky Nature Reserve, cordon "52 km" $\left(54.087^{\circ} \mathrm{N} 126.871^{\circ} \mathrm{E}\right), 5$ and $10 . \mathrm{VII} .1981, \mathrm{~A}$ Ozerov, A. Shatalkin ( $\sigma^{7}, 1$, ZMUM); Khabarovsk Krai: River Gobilli $\left(49.22^{\circ} \mathrm{N} 138.25^{\circ} \mathrm{E}\right), 8 . \mathrm{VI} .2014$, N. Vikhrev (1 + , ZMUM); Primorsky Krai: Kedrovaya Pad' Nature Reserve $\left(43.104^{\circ} \mathrm{N} 131.512^{\circ} \mathrm{E}\right), 10 . \mathrm{V} .1962$, Rasnitsyn (1 $\sigma^{7}$, ZISP); Andreevka (ca. $42.64^{\circ} \mathrm{N} 131.13^{\circ} \mathrm{E}$ ), 25-30.VI.2014, N. Vikhrev (4 ㅇ, ZMUM); Sikhote-Alin Nature Reserve, Blagodatnoe $\left(44.975^{\circ} \mathrm{N}\right.$ 136.526 $6^{\circ} \mathrm{E}$ ), 1-6.VI.2018, M. Sergeev (3 $\sigma^{7} \sigma^{7}$, ZMUM).

DIAGNOSTIC DESCRIPTION. Male. Female. Body-length 5.1-6.4 mm. Head. Frontal vitta yellow, matt; fronto-orbital plate blackish, greyish dusted. Face, parafacial and gena yellowish white. Postcranium greyish dusted, blackish in upper half and whitish in lower half in male and yellow in upper half and whitish in lower half in female. 3 orbital and 3-4 frontal setae present. Antenna black as a rule completely in male; scapus and pedicel usually yellow in female. Postpedicel approximately 2.5 times as long as wide. Arista nearly bare. Palpus pale yellow. Thorax yellow in ground color, greyish dusted; anepisternum, anepimeron and mediotergite in some specimens partly blackish. Acrostichals setulose in two rows, dorsocentrals $3+3$ (including scapular seta), intra-alars $1+2$, supra-alars $1+2,2$ postpronotals. Scutellum with a pair of strong discal setae and a pair of apical setulae. Legs yellow completely. Wing tinged with light brownish; veins brown. Vein $\mathrm{R}_{1}$ setulose on apical third of dorsal surface. Abdomen blackish with greyish pollen; female tergite 6 brownish. Male sternite 4 rectangular, longer than wide. Male sternite 5, epandrium and surstyli as in Figs 125-127.

DISTRIBUTION. Russia: Amur Oblast, Khabarovsk Krai, Primorsky Krai.

\section{Cordilura ustulata (Zetterstedt, 1838)}

Figs 5, 128-130.

ustulata Zetterstedt, 1838: 727 (Cordylura). Type-locality: "Lapponia Umensis... Tornensis... Nordlandiae" (Norway, Sweden).

melanacra Loew, 1873: 247 (Cordylura). Type-locality: "Rosenthal" [now Rózanka, part of Międzylesie, near Kłodzko (Poland)].

REMARKS. The species was recorded from Siberia [Ozerov, Barkalov, 2014: 561], Amur Oblast, Primorsky Krai and Sakhalin Oblast [Ozerov, Krivosheina, 2014: 212]. Noted by Gorodkov [1986: 17] for north of European part and Siberia without indicating specific locality.

MATERIAL EXAMINED. Amur Oblast: River Nyukzha $\left(56.574^{\circ} \mathrm{N} 121.652^{\circ} \mathrm{E}\right), 18 . \mathrm{VI} .1976$, Soboleva (1 フ, 3 우, IBSS); Arkhangelsk Oblast: Nenetsky Nature Reserve, cordon "Bol'shoy Gusinets" (68.175 $\left.\mathrm{N}, 53.645^{\circ} \mathrm{E}\right), 10-11 . \mathrm{VII} .2008$, N.E. Vikhrev, A.L. Ozerov ( $5 \sigma^{7} \sigma^{7}, 6$ 우, ZMUM); the lower reaches of the River Pechora $\left(68.334^{\circ} \mathrm{N}, 53.304^{\circ} \mathrm{E}\right), 11 . \mathrm{VII} .2008$, A.L. Ozerov (1 $\sigma^{7}$, ZMUM); $73 \mathrm{~km} \mathrm{NW}$ of Nar'yan-Mar (ca. $67.981^{\circ} \mathrm{N} 51.608^{\circ} \mathrm{E}$ ), 3.VIII.1978, Gorodkov (1 $\sigma^{7}$, ZISP); Chelyabinsk Oblast: Zlatoust Miass $\left(55.166^{\circ} \mathrm{N} 59.666^{\circ} \mathrm{E}\right), 2$.VI.1936, Zhelokhovtsev (2 9 , ZMUM); Krasnoyarsk Krai: Taymyr, Taymyrsky Nature Reserve, cordon Ary-Mas $\left(72.5^{\circ} \mathrm{N}, 101.94^{\circ} \mathrm{E}\right), 14 \mathrm{~m}, 8$.VII.2010, O. Khruleva $\left(1 \sigma^{7}\right.$, ZMUM); Khatanga $\left(71.975^{\circ} \mathrm{N}, 102.482^{\circ} \mathrm{E}\right), 11-$ 12.VII.1970, V. Zherikhin, A. Ponamorenko, A. Rasnitsyn, I. Sukacheva (1 $\sigma^{7}$, ZMUM); Kryuchkovo station $\left(56.096^{\circ} \mathrm{N} 62.109^{\circ} \mathrm{E}\right)$, 14-23.VII.2009, K. Tomkovich (1 +, ZMUM); Medvezhiy $\left(69.284^{\circ} \mathrm{N} 88.148^{\circ} \mathrm{E}\right)$, Norilsk env., 6.VII.1967, Gorodkov (1 $\bigcirc^{7}$, ZISP); Leningrad Oblast: Yashchera $\left(58.894^{\circ} \mathrm{N} 29.820^{\circ} \mathrm{E}\right)$, 8.VI.1958, A. Stackelberg (1 + , ZISP); Moscow Oblast: Dmitrov env. $\left(56.316^{\circ} \mathrm{N} 37.725^{\circ} \mathrm{E}\right), 19 . \mathrm{V} .2006$, N.Vikhrev (1 9 , ZMUM); Murmansk Oblast: Tumannyy $\left(68.883^{\circ} \mathrm{N} 35.693^{\circ} \mathrm{E}\right), 14$.VII.1974, Kasparyan (1 ऽ, ZISP); Vud'yavr (ca. $\left.67.64^{\circ} \mathrm{N} 33.644^{\circ} \mathrm{E}\right), 27 . \mathrm{VI}$. and 6.VII.1930, Fridolin ( $\sigma^{\top}, 1$, ZISP); Primorsky Krai: Lazovsky Nature Reserve, cordon "Korpad" $\left(43.252^{\circ} \mathrm{N} 134.126^{\circ} \mathrm{E}\right)$, 19-20.V.2007, V. Sidorenko (1 O, ZMUM); Vinogradovka $\left(43.762^{\circ} \mathrm{N} 132.951^{\circ} \mathrm{E}\right), 20 . \mathrm{VII} .1929$, Kirichenko (1 $\sigma^{7}$, ZISP); Tyumen' Oblast: Labytnangi env. (66.661 $\left.{ }^{\circ} \mathrm{N} 66.394^{\circ} \mathrm{E}\right), 17$. VII.1971, Ol'shvang (4 $\bigcirc^{7} \sigma^{7}, 6$ 우, ZISP); Neroyka (ca. $64.57^{\circ} \mathrm{N} 59.67^{\circ} \mathrm{E}$ ), $600 \mathrm{~m}, 17 . \mathrm{VI} .1989$, Malozemov (1 $\sigma^{7}$, ZISP); same place, $500 \mathrm{~m}$, 12-15.VII.1990, Malozemov (1 + , ZISP); Sakhalin Oblast: Shikotan I., Tserkovnaya Bay $\left(43.75^{\circ} \mathrm{N} 146.70^{\circ} \mathrm{E}\right), 2-8$.VI.2012, Yu. Sundukov (1 $\sigma^{7}$, ZMUM); Yakutia: Pokhodsk $\left(69.079^{\circ} \mathrm{N}\right.$ $\left.160.961^{\circ} \mathrm{E}\right)$, 5.VII.1980, Bagachanova (1 $\bigcirc^{7}$, ZISP).

DIAGNOSTIC DESCRIPTION. Male. Female. Body-length 5.6-7.0 mm. Head. Frontal vitta black or dark brown, sometimes reddish yellow apically; fron- 

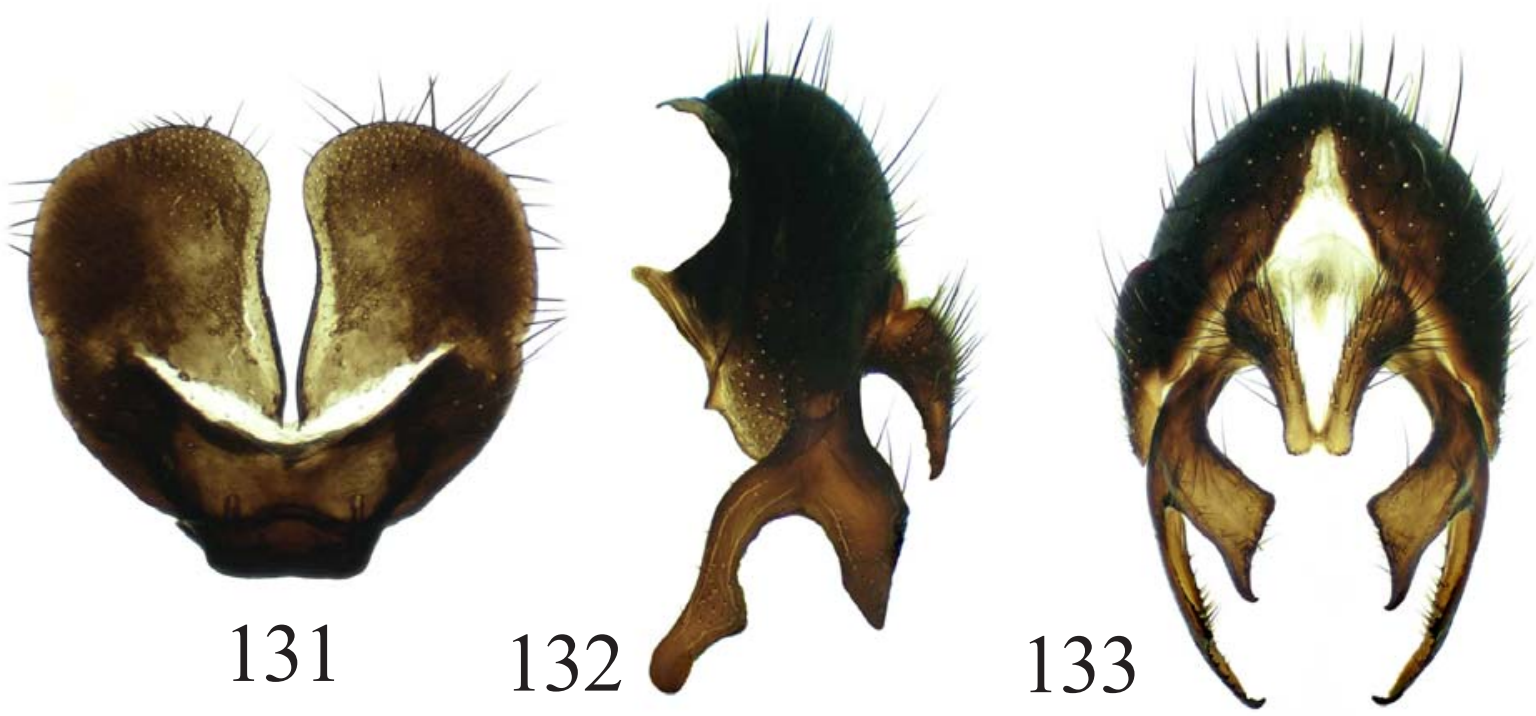
view.

Figs 131-133. Cordilura zaitzevi Gorodkov: 131 - male sternite 5; 132 - epandrium and surstyli, lateral view; 133 - same, dorsal

Рис. 131-133. Cordilura zaitzevi Gorodkov: 131 - стернит 5 самца; 132 - эпандрий и сурстили, сбоку; 133 - то же, сверху.

to-orbital plate black, subshining or greyish dusted. Face and parafacial yellow with whitish reflection. Gena from yellow to brown. Postcranium black, delicately greyish dusted. 3 orbital and 3-5 frontal setae present. Antenna blackish. Postpedicel approximately 1.5 times as long as wide. Arista nearly bare (Fig. 5). Palpus yellow. Thorax black, delicately greyish dusted, only scutum usually mostly subshining. Acrostichals absent, dorsocentrals $3+3$ (including scapular seta), intra-alars $0+(0-2)$ (usually absent), supra-alars $1+2,2$ postpronotals. Scutellum with a pair of strong discal and a pair of strong apical setae. Legs subshining. Femora black, fore tibia and tarsi of all legs yellow, mid and hind tibiae from yellow to black. Wing tinged with yellowish; veins yellowish. Vein $\mathrm{R}_{1}$ bare or with several setulae on apical third of dorsal surface. Apex of wing usually darkened. Abdomen black, delicately greyish dusted. Male sternite 4 rectangular, longer than wide. Male sternite 5, epandrium and surstyli as in Figs 128-130.

DISTRIBUTION. Russia: Amur Oblast, Arkhangelsk Oblast, Chelyabinsk Oblast, Krasnoyarsk Krai, Leningrad Oblast, Moscow Oblast, Murmansk Oblast, Primorsky Krai, Tyumen' Oblast, Sakhalin Oblast, Yakutia. — Europe, Mongolia [Šifner, 1975: 220]; North America.

\section{Cordilura zaitzevi Gorodkov, 1974} Figs 131-134.

zaitzevi Gorodkov, 1974: 386 (Cordilura). Type-locality: Songino, 1300 m, Central aimak (Mongolia).

REMARK. The species was recorded in Russia from Buryatia and Yakutia [Gorodkov, 1974: 386], from Amur Oblast and Primorsky Krai [Ozerov, Krivosheina, 2014: 212] and from Yakutia [Bagachanova et al., 2016: 779].
MATERIAL EXAMINED. Altai: $10 \mathrm{~km}$ E of Cheke-Taman Pass, River Bol'shoy Il'gumen' $\left(50.613^{\circ} \mathrm{N} 86.469^{\circ} \mathrm{E}\right)$, 28.VII.2006, A. Ovchinnikov $\left(1\right.$ ㅇ, ZISP); Kuray env. $\left(50.233^{\circ} \mathrm{N} 87.930^{\circ} \mathrm{E}\right)$, 25.VII.2006, A. Ovchinnikov (4 우, ZISP); Amur Oblast: Blagoveshchensk $\left(50.280^{\circ} \mathrm{N} 127.535^{\circ} \mathrm{E}\right), 17 . \mathrm{VIII} .1982$, A. Leley (1 ㅇ, ZISP); Zeya Town $\left(53.748^{\circ} \mathrm{N} 127.261^{\circ} \mathrm{E}\right), 18$ and 20.VII.1982, A. Ozerov, O. Gorbunov (2 $0^{7} 0^{7}, 1$, ZMUM); Khakassia: Borets $\left(54.46^{\circ} \mathrm{N}\right.$ $\left.90.36^{\circ} \mathrm{E}\right)$, 29.VI.2011, K. Tomkovich (2 $0^{7} \sigma^{7}$, ZMUM); Novosibirsk Oblast: Koynikha $\left(54.568^{\circ} \mathrm{N} 83.252^{\circ} \mathrm{E}\right), 13$.VIII.1977, Gorodkov (4 $\bigcirc^{7} \bigcirc^{7}, 4$ 우, ZISP); Primorsky Krai: River Bol'shaya Ussurka, $20 \mathrm{~km} \mathrm{NW}$ of Mel'nichnoe $\left(45.548^{\circ} \mathrm{N} 135.347^{\circ} \mathrm{E}\right), 2$.VIII.1986, A. Leley $\left(1 \bigcirc^{7}\right.$, IBSS); Kamenushka $\left(43.634^{\circ} \mathrm{N} 132.222^{\circ} \mathrm{E}\right), 9$ and 11.IX.1983, A.L. Ozerov (3 O $^{7} \sigma^{7}$, ZMUM); Shkotovskoe plateau (ca. $\left.43.4589^{\circ} \mathrm{N} 132.6324^{\circ} \mathrm{E}\right), 25$. VIII.1962, Nartshuk (1 +, ZISP); Yakutia: Pokrovsk $\left(61.467^{\circ} \mathrm{N} 129.101^{\circ} \mathrm{E}\right), 21$. VII.1986, Makarkin (1 $\mathrm{O}^{7}$, IBSS); Mikhaylovka, $60 \mathrm{~km}$ NE of Amga $\left(61.214^{\circ} \mathrm{N}\right.$ 132.681 $\left.{ }^{\circ} \mathrm{E}\right), 23$. VII.1986, Sivtsev ( $2 \mathrm{O}^{7} \mathrm{O}^{7}, 3$ 우, ZISP); same place, 17.VII.1987, Bagachanova (12 $\mathrm{O}^{7} \sigma^{7}, 14$ 우, ZISP); same place, 5.VIII.1987, Zlobin (2 웅, ZISP); Alagar-Khoy, $18 \mathrm{~km} \mathrm{~N}$ of Tyungyulyu $\left(62.346^{\circ} \mathrm{N} 130.649^{\circ} \mathrm{E}\right), 21$.VII.1985, Bagachanova (1 $0^{7}, 2$ 우

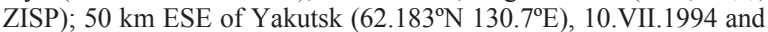
6.VII.1996, Bagachanova (1 Oొ, 2 우, ZISP); Usun-Kyuel' env. $\left(62.870^{\circ} \mathrm{N} 130.832^{\circ} \mathrm{E}\right), 18$. VII.1997, Bagachanova (1 + , ZISP); Yakutsk env., Chochur-Muran $\left(62.034^{\circ} \mathrm{N} 129.618^{\circ} \mathrm{E}\right)$, 2.VII.2008, A. Ovchinnikov (1 $\sigma^{7}, 1$ + , ZISP); Yakutsk, Botanical Garden $\left(62.023^{\circ} \mathrm{N} 129.614^{\circ} \mathrm{E}\right), 14$. VII.1976, V. Kovalev (1 $\sigma^{7}$, ZISP); same place, 18.VII.1974, Nartshuk (3 $\sigma^{7} \sigma^{7}, 1$ \%, ZISP); Yakutsk $\left(62.023^{\circ} \mathrm{N}\right.$ 129.614E), 10.VIII.1987, Zlobin (1 $\sigma^{7}$, ZISP).

DIAGNOSTIC DESCRIPTION. Male. Female. Body-length 5.8-7.1 mm. Head. Frontal vitta black with weak whitish reflection; fronto-orbital plate black, greyish dusted. Face and parafacial yellowish, with whitish reflection. Gena blackish. Postcranium black completely, greyish dusted. 3 orbital and 2-3 frontal setae present. Scapus and pedicel from yellowish to blackish; postpedicel blackish, approximately 2 times as long as wide. Arista plumose, the longest hairs approximately equal to the width of postpedicel. Palpus yellow or brownish (female). Thorax black, delicately greyish dusted. Acrostichals absent, dorsocentrals $3+3$ (including scapular 
seta), intra-alars $0+(0-2)$, supra-alars $1+2,2$ postpronotals. Scutellum with a pair of strong discal setae only; apical scutellar setae as setulae. Legs greyish dusted. Fore coxa white. Femora of all legs black; tibiae and tarsi of all legs yellow. Wing tinged with brownish; veins dark yellow. Vein $\mathrm{R}_{1}$ bare or with 1-3 setulae on apical third of dorsal surface. Abdomen black, greyish dusted. Male sternite 4 triangular (Fig. 15). Male sternite 5, epandrium and surstyli as in Figs 131-133.

Photo of female ovipositor in lateral view was given by Ovchinnikov [2009: 632].

DISTRIBUTION. Russia: Altai, Amur Oblast, Buryatia, Khakassia, Novosibirsk Oblast, Primorsky Krai, Yakutia. - Kazakhstan and Mongolia [Gorodkov, 1974: 386].

\section{Key to the SPECIES of CordiluRa FAllén of Russia}

1. Scutellum with a pair of strong discal and a pair of strong apical setae (e.g., Fig. 13)

- Scutellum with a pair of strong discal setae only; apical scutellar setae as setulae or absent (e.g., Fig. 12) ...... 15

2. Palpus yellow ................................................................. 3

- Palpus black or dark brown

3. Arista plumose, the longest hairs approximately equal to the width of postpedicel. Frontal vitta yellow. Male sternite 5, epandrium and surstyli as in Figs 84-86 C. picticornis (Loew)

- Arista nearly bare (Fig. 5). Frontal vitta black. Apex of wing usually darkened. Male sternite 5, epandrium and surstyli as in Figs 128-130 ...... C. ustulata (Zetterstedt)

4. Postpedicel with 1-2 additional long setae externally from plumose arista (Fig. 6). Male sternite 5, epandrium and surstyli as in Figs 45-47 .......... C. ciliata (Meigen)

- Postpedicel without additional setae .....

5. Legs, including tarsi black completely. Male sternite 5, epandrium and surstyli as in Figs 39-41

. C. atrata (Zetterstedt)

- At least fore tibiae and tarsi yellow

6. Tarsomeres 2-4 darkened basally of at least the fore leg (e.g., Fig. 11)

— Tarsi ventrally unicolor, yellow or brownish .................... 8

7. Arista pubescent, the longest hairs approximately equal to $1 / 3-1 / 2$ width of postpedicel (Fig. 4). Male sternite 5, epandrium and surstyli as in Figs 27-29

C. aemula (Collin)

- Arista plumose, the longest hairs approximately equal to the width of postpedicel (Fig. 3). Male sternite 5, epandrium and surstyli as in Figs 104-106

C. rufipes (Meigen)

8. Arista plumose or pubescent, longest hairs exceeding greatest diameter of arista (e.g., Figs 3, 4, 6) ............. 9

- Arista nearly bare, longest hairs not exceeding greatest diameter of arista (e.g., Fig. 5) .................................... 12

9. Scutum with 2 postsutural intra-alar setae; presutural acrostichal hairs present ........................................... 10

- Scutum with 1 postsutural intra-alar seta only; presutural acrostichal hairs absent. Male sternite 5, epandrium and surstyli as in Figs 24-26 ............. C. aberrans (Becker)

10. Presutural intra-alar seta present. Hind tibia yellow ...... 11

- Presutural intra-alar seta absent. Hind tibia black or dark brown. Male sternite 5, epandrium and surstyli as in Figs 101-103
11. Tarsi ventrally yellow. Male sternite 5, epandrium and surstyli as in Figs 90-92 ............... C. pudica (Meigen)

- Tarsi ventrally dark brown (Fig. 10). Male sternite 5, epandrium and surstyli as in Figs 69-71 .....

C. monochroma Ozerov et Krivosheina

12. Legs shining. Frontal vitta yellow ................................. 13

- Legs greyish dusted. Frontal vitta dark brown. Male sternite 5, epandrium and surstyli as in Figs 122-124 ..

C. socialis Becker

13. 3 orbital setae present .................................................. 14

- 2 orbital setae present. Male sternite 5, epandrium and surstyli as in Figs 54-56 .................. C. grunini Ozerov

14. Male sternite 5, epandrium and surstyli as in Figs 48-50 C. fulvifrons Ozerov

- Male sternite 5, epandrium and surstyli as in Figs 87-89 C. proboscidea (Zetterstedt)

15. Arista plumose or pubescent, longest hairs exceeding greatest diameter of arista (e.g., Figs 3, 4, 6) ........... 16

- Arista nearly bare, longest hairs not exceeding greatest diameter of arista (e.g., Fig. 5) .................................... 30

16. Fore coxa black ................................................... 17

— Fore coxa white or yellowish ......................................... 21

17. Presutural supra-alar seta usually absent or setulose; intra-alar setae absent. Vein $\mathrm{R}_{1}$ bare on apical third of dorsal surface. Male sternite 5, epandrium and surstyli as in Figs 60-62 ...................... C. kakaberrans Ozerov

- Presutural supra-alar seta present; $(0-1)+(1-2)$ intra-alar setae present. Vein $\mathrm{R}_{1}$ setulose on apical third of dorsal surface (e.g., Fig. 19) ................................................ 18

18. Tibiae and tarsi of all legs yellow ................................. 19

- Tibiae and tarsi of all legs black. Male sternite 5, epandrium and surstyli as in Figs 81-83

C. picipes (Meigen)

19. Presutural acrostichal hairs present .............................. 20

- Presutural acrostichal hairs absent. Male sternite 5, epandrium and surstyli as in Figs 63-65

C. kosterini Ozerov et Krivosheina

20. Male sternite 5, epandrium and surstyli as in Figs 107109 ........................................ C. sagittifera Gorodkov

- Male sternite 5, epandrium and surstyli as in Figs 57-59 C. impudica Rondani

- Male sternite 5, epandrium and surstyli as in Figs 113115 .......................................... C. sibirica Gorodkov

21. Mid and hind femora bicolor: black in apical part and yellow in basal half or third. Male sternite 5, epandrium and surstyli as in Figs 42-44 ...... C. bicoloripes Ozerov

- Mid and hind femora yellow completely (sometimes darkened dorsally) or black completely ..................... 22

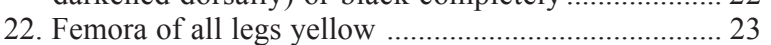

— Femora of all legs black .................................................. 28

23. Postpedicel bicolor: yellow basally and blackish in apical part. Male sternite 5, epandrium and surstyli as in Figs 51-53

C. fuscipes (Zetterstedt)

- Postpedicel unicolor: completely yellow, black or dark brown ..................................................................... 24

24. Postpedicel yellow, approximately 1.5-2 times as long

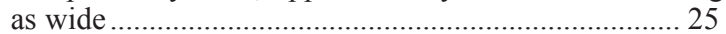

- Postpedicel black, approximately 3-3.5 times as long as wide (Fig. 7). Male sternite 5, epandrium and surstyli as in Figs $30-32$......................... C. albilabris (Fabricius)

25. Thorax black completely. Male sternite 5, epandrium and surstyli as in Figs 98-100.

C. richterae Ozerov et Krivosheina

- Thorax with pleural sclerites mostly or completely pale yellow 
26. Wing with a distinct brown spot or shadow apically (e.g., Fig. 20) .. 27

- Wing tinged with brownish, but without a distinct brown spot or shadow apically. Male sternite 5, epandrium and surstyli as in Figs 33-35 C. albipes (Fallén)

27. Male sternite 5, epandrium and surstyli as in Figs 75-77 C. nubecula (Sasakawa)

- Male sternite 5, epandrium and surstyli as in Figs 116118 ..................... C. sidorenkoi Ozerov et Krivosheina

- Male sternite 5, epandrium and surstyli as in Figs 110 112 ........................................... C. shatalkini Ozerov

— Epandrium and surstyli as in Figs 96, 97

28. Scutum with 1 postpronotal seta C. remota Ozerov

.

Scutum with 2 postpronotal setae. Male sternite 5, epandrium and surstyli as in Figs 131-133

C. zaitzevi Gorodkov

29. Femora subshining. Male sternite 5, epandrium and surstyli as in Figs 93-95 ......................... C. remmi Elberg

- Femora grey dusted. Male sternite 5, epandrium and surstyli as in Figs 72-74

C. negrobovi Ozerov et Krivosheina

30. Postpedicel yellow completely or blackish in apical half on the outside only ................................................... 31

— Postpedicel blackish completely .................................... 34

31. Vein $R_{2+3}$ with a brown spot apically, intersection of vein $\mathrm{M}$ and crossvein dm-cu with a brown spot, apex of wing darkened (Fig. 18). Hind tibia with apical ventral seta ...

C. nartshukae Ozerov et Krivosheina

- Wing clear, without dark spots. Hind tibia without apical ventral seta

32

32. Postpedicel yellow basally and blackish in apical half on the outside. Frontal vitta yellow apically and blackish basally. Scutum black in male and yellow with blackish stripes distally from each dorsocentral line in female. Male sternite 5, epandrium and surstyli as in Figs 66-68 C. krocha Ozerov

- Postpedicel and frontal vitta yellow completely ......... 33

33. Scutum reddish yellow, with blackish stripes distally from each dorsocentral line. 2 postsutural intra-alar setae present. Male sternite 5, epandrium and surstyli as in Figs 119-121 C. sifneri Ozerov

- Scutum reddish yellow, unicolor. Postsutural intra-alar setae absent .................. C. amurensis Ozerov (female)

34. 1 postpronotal seta present. Scutum unicolor: reddish yellow or blackish

35

- 2 postpronotal setae present. Scutum reddish yellow, with blackish stripes distally from each dorsocentral line. Male sternite 5, epandrium and surstyli as in Figs 125 127 ......................................... C. tartariana Ozerov

35. Scutum reddish yellow. Male sternite 5, epandrium and surstyli as in Figs 36-38 ..... C. amurensis Ozerov (male)

- Scutum blackish. Male sternite 5, epandrium and surstyli as in Figs $78-80$.................... Cordilura nubilosa, sp.n.

Acknowledgements. We are very grateful to Dr. Olga Ovchinnikova and Mrs. Galina Suleymanova for the help during our work in ZISP with the material of Cordilura. We are grateful to Dmitry I. Gavryushin (Moscow) for the photos of adults Cordilura rufipes (Meigen) and Cordilura picipes (Meigen).

The investigation was fulfilled within the state project No AAAA-A18-118042490060-1 (M.G. Krivosheina) and the state project No AAA-A15-116021660077-3 (A.L. Ozerov).

\section{References}

Bagachanova A.K., Ovchinnikov A.N., Ozerov A.L. 2016. On the Fauna of Scathophagidae (Diptera) of Yakutia // Entomological Review. Vol.96. No.6. P.775-785.

Becker T. 1894. Dipterologische Studien. I. Scatomyzidae // Berliner Entomologische Zeitschrift. Bd.39. H.1. S.77-196.

Collin J.E. 1958. A short synopsis of the British Scatophagidae // Transactions of the Society for British Entomology. Vol.13. P.37-56.

Cumming J.M., Wood D.M. 2009. Adult morphology and terminology // Brown B.V., Borkent A., Cumming J.M., Wood D.M., Woodley N.E., Zumbado M. (eds.). Manual of Central American Diptera. Vol. 1. National Research Council Press, Ottawa. P. 9-50

Elberg K. 1972. Cordilura remmi sp. n. a new species of Scatophagidae (Diptera) from the Far East // Acta et Commentationes Universitatis Tartuensis, Zoologia - Alaseid Toid. Vol.6. P.9196.

Engelmark R. 1999. Dungflies (Diptera: Scathophagidae) collected by the Swedish-Russian tundra ecology expedition '94, with the description of two new species; Nanna indotatum and Cochliarium sibiricum // Entomologisk Tidskrift. Arg.120. Häft 4. P.157-167.

Fabricius J.C. 1805. Systema atliatorum secundum ordines, genera, species adiecta synonymis, locis, observationibus, descriptionibus. Brunsvigae, xiv $+15-372+30 \mathrm{pp}$.

Fallén C.F. 1810. Specimen entomologicum novam Diptera disponendi methodum exhibens. Lundae: Litteris Berlingianis. $26 \mathrm{pp}$.

Fallén C.F. 1819. Scatomyzides. Lundae. 10 pp.

Ferrar P. 1987. A guide to the breeding habits and immature stages of Diptera Cyclorrhapha. Entomonograph. Vol.8. Part.1-2. 907 pp.

Gorodkov K.B. 1970. [Family Scathophagidae (Cordyluridae, Scatomyzidae, Scopeumatidae)] // Bei-Bienko G.Ya. (ed.). Key to the insects of the European part of the USSR. Vol.5. Diptera, Siphonaptera. Part 2. P.440-458 [in Russian].

Gorodkov K.B. 1974. [Scatophagidae (Diptera) of Mongolian People's Republic] // Nasekomye Mongolii. T.2. P.380-395 [in Russian].

Gorodkov K.B. 1986. Family Scathophagidae // Soós Á., Papp L. (eds.). Catalogue of Palaearctic Diptera. Vol.11. ScathophagidaeHypodermatidae. Akadémiai Kiadó, Budapest. P.11-41.

Hackman W. 1956. The Scatophagidae (Dipt.) of Eastern Fennoscandia // Societas pro Fauna et Flora Fennica. Fauna fennica II. Helsingforsiae. $67 \mathrm{pp}$.

Hendel F. 1930. Entomologische Ergebnisse der schwedischen Kamtchatka-Expedition 1920-1922. 28. Diptera Brachycera 2. Fam. Cordyluridae und Dryomyzidae // Arkiv för Zoologi. Bd.21A. No.18. S.1-12.

Humala A.E., Polevoi A.V. 2009. On the Insect fauna of south-east Karelia // Trudi Karelskogo nauchnogo centra RAN. Vol.4. P. 53-75.

Iwasa M. 2020. Contribution to the knowledge of the genus Cordilura Fallén (Diptera, Scathophagidae) from Japan, with descriptions of three new species // Zootaxa. Vol.4748. No.3. P.471-484.

James M.T. 1955. The genus Cordilura in America North of Mexico // Annals of the Entomological Society of America. Vol.48. No.1-2. P.84-100.

Khaghaninia S., Gharajedaghi Y. 2014. Dung flies fauna (Diptera: Scathophagidae) in East Azerbaijan province of Iran, with eight new records // Journal of Crop Protection. Vol.3. No.3. P.389-396.

Loew H. 1864. Acht neue Cordylura-Arten // Wiener Entomologische Monatschrift. Bd.8. S.17-26.

Loew H. 1873. Beschreibungen europäischen Dipteren. Dritter Band. Systematische Beschreibung der bekannten europäischen zweiflügeligen Insecten. Von Johann Wilhelm Meigen. Zehnter Theil oder vierter Supplementband. Schmidt. Halle. viii +320 S.

McAlpine J.F. 1981. Morphology and terminology-adults // McAlpine J.F., Peterson B.V., Shewell G.E, Teskey H.J., Vo- 
keroth J.R., Wood D.M. (Coordinators). Manual of Nearctic Diptera. Vol.2. Research Branch. Agriculture Canada. Monograph 27, Ottawa. P.9-63

Meigen J.W. 1826. Systematische Beschreibung der bekannten europäischen zweiflügeligen Insecten. Fünfter Theil. [5]. Hamm. $412 \mathrm{~S}$.

Meigen J.W. 1838. Systematische Bescreibung der bekannten europaischen zweiflügeligen Dipteren. Vol.7. Hamm. 434 S.

Neff S.E., Wallace J.B. 1969. Observations on the immature stages of Cordilura (Achaetella) deceptiva and C. (A.) varipes // Annals of the Entomological Society of America. Vol.62. P.775-785.

Ovchinnikov A.N. 2004. Data on the fauna of Scathophagidae (Diptera) of the Volga Region // Russian Entomological Journal. Vol.12. No.4(2003). P.421-423.

Ovchinnikov A.N. 2009. The Ovipositor Morphology in the Members of the Family Scathophagidae (Diptera) with Reference to Their Biology // Entomological Review. Vol.88. No.2. P.314-328.

Ozerov A.L. 1997. [Five new species of the genus Cordilura (Diptera, Scathophagidae) from the Far East of Russia] // Zoologicheskiy zhurnal. Vol.76. No.12. P.1424-1429 [in Russian].

Ozerov A.L. 2007a. On Scathophagidae species (Insecta: Diptera), described by F. Hendel from the materials of the Swedish Kamchatka expedition 1920-1922 // Far Eastern Entomologist. No.174. P.5-8

Ozerov A.L. 2007b. Four new species of dung flies (Diptera: Scathophagidae) from Russian Far East // Russian Entomological Journal. Vol.16. No.1. P.123-126.

Ozerov A.L. 2009. Fam. Scathophagidae. P.379. Storozhenko S.Yu. et al. (Eds). Insects of Lazovsky Nature Reserve. Vladivostok: Dalnauka. 464 pp. [in Russian].

Ozerov A.L., Barkalov A.V. 2014. On the fauna of Scathophagidae (Diptera) of Taimyr Peninsular (Russia: Krasnoyarskiy Kray) // Euroasian Entomological Journal. Vol.13. No.6. P.560-565.

Ozerov A.L., Krivosheina M.G. 2012a. New species of Sepsidae and Scathophagidae (Diptera) from Vietnam // Russian Entomological Journal. Vol.21. No.3. P.331-335.

Ozerov A.L., Krivosheina M.G. 2012b. To knowledge of the genus Cordilura Fallén (Diptera, Scathophagidae), with description of a new species from the Russian Far East // Far Eastern Entomologist. No.249. P.1-4.

Ozerov A.L., Krivosheina M.G. 2013. A new species of the genus Cordilura Fallén (Diptera, Scathophagidae) from Vietnam // Far Eastern Entomologist. No.265. P.16-19.

Ozerov A.L., Krivosheina M.G. 2014. To the fauna of dung flies (Diptera: Scathophagidae) of Russian Far East // Russian Entomological Journal. Vol.23. No.3. P.203-222.
Ozerov A.L., Krivosheina M.G. 2015. Two new species of the genus Cordilura Fallén, 1810 (Diptera, Scathophagidae) from Russian Far East // Zootaxa. Vol.4040. No.4. P.489-493.

Ozerov A.L., Krivosheina M.G. 2017. Two new species of the genus Cordilura Fallén,1810 (Diptera: Scathophagidae) from Buryatia and Primorsky Krai (Russia) // Far Eastern Entomologist. No.335. P.1-6.

Robineau-Desvoidy J.B. 1830. Essai sur les Myodaires. Paris. Mémoires présentés par divers Savans à l'Académie Royale des Sciences de 1'Institut de France. Tome.2. P.1-813.

Rondani C. 1867. Scatophaginae Italicae collectae distinctae et in ordinem dispositae. Dipterologiae Italicae prodromus. Pars VII, Fasc I. // Atti della Società Italiana di Scienze Naturali. Vol.10. P. 85-135.

Sasakawa M. 1986. Two new species of the genus Cordilura Fallén (Diptera, Scatophagidae) from Japan // Proceedings of the Japanese Society of Systematic Zoology. No.33. P.40-44.

Šifner F. 1975. Scatophagidae (Diptera) de Mongolie // Annales Historico-Naturales Musei Nationalis Hungarici. Vol.67. P.219227.

Šifner F. 2008. A catalogue of the Scathophagidae (Diptera) of the Palaearctic region, with notes on their taxonomy and faunistics // Acta Entomologica Musei Nationalis Pragae. Vol.48. No.1. P.111-196.

Stuckenberg B.R. 1999. Antennal evolution in the Brachycera (Diptera), with a reassessment of terminology relating to the flagellum // Studia Dipterologica. Vol.6. S.33-48.

Sun X. 1993. [Notes on the genus Cordilura Fallén from China (Diptera, Scathophagidae)] // Sinozoologia. Vol.5. No.10. P.437-440 [in Chinese with English summary].

Vockeroth J.R. 1965. Subfamily Scatophaginae // Stone A. et al. (eds.). A catalog of the Diptera of America north of Mexico. United States Department of Agriculture, Agriculture Handbook. No.276. P.826-842.

Vockeroth J.R. 1987. 103. Scathophagidae // McAlpine J.F. et al. (eds.). Manual of Nearctic Diptera. 2. Ottawa: Agriculture Canada, Research Branch. Monograph 28. P.1085-1097.

Wallace J.B., Neff S.E. 1971. Biology and immature stages of the genus Cordilura (Diptera: Scathophagidae) in the eastern United States // Annals of the Entomological Society of America. Vol.64. No.6. P.1310-1311.

Zetterstedt J.W. [1838]. Sectio tertia. Diptera // Insecta Lapponica.' 1840 '. Lipsiae: Leopold Voss. P.477-868.

Zetterstedt J.W. 1846. Diptera Scandinaviae disposita et descripta. Tomus quintus. Ex officina lundbergiana, Lundae [= Lund]. P.1739-2162. 